Алгебра и анализ

Том. 15 (2003), вып. 6
St. Petersburg Math. J.

Vol. 15 (2004), No. 6, Pages 875-913 S 1061-0022(04)00837-4

Article electronically published on November 15, 2004

\title{
ON THE NONSTATIONARY MAXWELL SYSTEM IN DOMAINS WITH EDGES
}

\author{
S. MATYUKEVICH
}

\section{INTRODUCTION}

In this paper, we study the behavior of solutions of the nonstationary Maxwell system near singularities of the boundary. As models, we consider a simply connected cone $\mathcal{K} \subset \mathbb{R}^{3}$ smooth outside its vertex, the wedge $\mathbb{D}=\mathbb{K} \times \mathbb{R}$, where $\mathbb{K}$ is a plane cone, a bounded domain $G \subset \mathbb{R}^{3}$ with a conical point, and the waveguide $\Sigma=\Omega \times \mathbb{R}$, where $\Omega$ is a plane domain with a corner point. The Maxwell system

$$
\left\{\begin{array}{c}
\partial \vec{E} / \partial t-\operatorname{curl} \vec{B}=-\vec{J}, \\
\partial \vec{B} / \partial t+\operatorname{curl} \vec{E}=-\vec{G}, \\
\operatorname{div} \vec{E}=\rho, \\
\operatorname{div} \vec{B}=\mu,
\end{array}\right.
$$

is endowed with the boundary conditions

$$
\vec{E} \times \vec{n}=0, \quad(\vec{B} \cdot \vec{n})=0,
$$

which correspond to the case of ideal conductive boundary; here $\vec{n}$ denotes the unit outward normal.

We use a method based on a priori estimates of solutions; this method was suggested in 11 for the wave equation with homogeneous Dirichlet boundary condition, and it was generalized in [2, 3, 4, 5] to various initial-boundary value problems related to the wave equation and to systems of the form $\partial_{t}^{2}-A(\partial)$, where $A(\partial)$ is a second order strongly elliptic operator. Briefly, the method can be described as follows. The Fourier transform $\mathcal{F}_{t \rightarrow \tau}$ yields a problem with parameter $\tau$. For a fixed parameter, this problem is elliptic. However, the problem is not elliptic with a parameter in the sense of [6]. A weak $a$ priori estimate can be proved by an "energy" argument in the entire domain under consideration; we call it the global energy estimate. Then, by using this estimate and the ellipticity of the operator $A(\partial)$, a more informative a priori estimate can be proved in a scale of weighted spaces; we call it the weighted combined estimate. The operators related to our problem are studied in the spaces dictated by the global and combined estimates. The asymptotics of solutions near singularities of the boundary and some formulas for the coefficients in the asymptotics can be obtained with the help of the theory of elliptic problems in domains with piecewise smooth boundary. At every step we trace the dependence on $\tau$. Using the inverse Fourier transform $\mathcal{F}_{\tau \rightarrow t}^{-1}$, we return to the initial nonstationary problem.

2000 Mathematics Subject Classification. Primary 35Q60.

Key words and phrases. Ideal conductive boundary, a priori estimates. 
The spatial part of the Maxwell system is not elliptic. However, acting as in [7] 8], we can augment it to an elliptic system:

$$
\left\{\begin{array}{c}
\partial \vec{E} / \partial t-\operatorname{curl} \vec{B}+\nabla h=\vec{f}_{1}, \\
\partial \vec{B} / \partial t+\operatorname{curl} \vec{E}+\nabla q=\overrightarrow{f_{2}}, \\
\partial h / \partial t+\operatorname{div} \vec{E}=g_{1}, \\
\partial q / \partial t+\operatorname{div} \vec{B}=g_{2} .
\end{array}\right.
$$

The corresponding augmented boundary conditions are of the form

$$
\vec{E} \times \vec{n}=0, \quad(\vec{B} \cdot \vec{n})=0, \quad h=0 .
$$

The augmented system will be written as $\partial v / \partial t+A(\partial) v=f$. The approach described above can be adapted to this system.

As an example, we briefly describe the results for the problem in a wedge $\mathbb{D}=\mathbb{K} \times \mathbb{R}$. For any vector-valued function $f=\left(\vec{f}_{1}, \vec{f}_{2}, g_{1}, g_{2}\right)$ such that $e^{-\gamma t} f \in L_{2}\left(\mathbb{D} \times \mathbb{R}_{t}\right)$, the augmented Maxwell system admits a unique solution $v=(\vec{E}, \vec{B}, h, q), e^{-\gamma t} v \in L_{2}\left(\mathbb{D} \times \mathbb{R}_{t}\right)$ with $\gamma>0$. For the solution $v$, we have an asymptotic expansion

$$
v(x, y, z, t)=c_{1}(z, t) r^{\pi / 2 \alpha-1} \Phi_{1}(\varphi)+h(x, y, z, t)
$$

in a neighborhood of the edge, where $(r, \varphi)$ are the polar coordinates on the plane $(x, y)$, $2 \alpha$ is the opening of $\mathbb{K}$, and $h$ is the remainder in the asymptotics. Moreover,

$$
c_{1}(z, t)=\int_{\mathbb{K}} \int_{\mathbb{R}} \int_{\mathbb{R}}\left\langle f(x, y, z-\xi, t-s), W_{1}(x, y, \xi, s)\right\rangle_{\mathbb{R}^{8}} d x d y d \xi d s,
$$

where $W_{1}$ denotes a specific solution of the system $\partial W_{1} / \partial t-A(\partial) W_{1}=0$ determined by an appropriate asymptotics near the edge. For the problem in $\mathcal{K}$ and in $\mathbb{D}$, such solutions are calculated explicitly (see Subsections 1.8, 2.7). Using explicit formulas for $W_{1}$ in $\mathcal{K}$, we observe in the coefficients of the asymptotics some phenomena related to the finite propagation speed of the electromagnetic waves (the vanishing of the coefficients before a perturbation meets the vertex of $\mathcal{K}$, and their smoothness after the perturbation leaves the vertex).

When proving the global energy estimate for the augmented Maxwell system, we face the necessity to view the spatial part $A(\partial)$ as a symmetric operator. For this reason, we are forced to impose certain special restrictions on the asymptotics near the singularities of the boundary for the functions in the domain of $A(\partial)$. This leads to a family of selfadjoint extensions of $A(\partial)$ (see Subsections 1.3, 2.5). The possibility of coming back to the initial nonaugmented Maxwell system depends on the choice of a selfadjoint extension (see Subsection 2.8). In particular, in a bounded domain with a conical point, the passage to the initial system is possible for a unique selfadjoint extension. To realize this passage, it suffices to take the right-hand side of the augmented system in the form $(-\vec{J},-\vec{G}, \rho, \mu)$, where $\vec{J}, \vec{G}, \rho, \mu$ are subject to the equations $\operatorname{div} \vec{J}+\partial \rho / \partial t=0$ and $\operatorname{div} \vec{G}+\partial \mu / \partial t=0$, and to the boundary condition $(\vec{G} \cdot \vec{n})=0$. Then $h$ and $q$ vanish, and the solution of the augmented system satisfies the usual Maxwell system (see Subsection 2.8). It turns out that the selfadjoint extension mentioned above coincides with the Maxwell operator studied in $[7$ for the stationary situation under much weaker restrictions on the smoothness of the boundary (see Subsection 2.8).

Besides the boundary conditions corresponding to the ideal conductive boundary, we consider the nonhomogeneous impedance conditions

$$
\vec{n} \times[\vec{B} \times \vec{n}]+\psi[\vec{E} \times \vec{n}]=[\vec{n} \times \vec{\Phi}],
$$


where the function $\psi$ characterizes the properties of the boundary. In the last Subsection 2.9 we briefly discuss the problem with the above nonhomogeneous conditions and prove a global energy estimate. We restrict ourselves to this, because the further arguments are similar to those in the first part.

Concerning papers devoted to the Maxwell system in domains with nonsmooth boundary, besides [7], we refer to the paper [10], where the singularities of the solutions of the stationary system were studied near edges and conical points. A short survey of publications on the regularity of solutions of the stationary Maxwell system can also be found in 10 . The model problem in a wedge $\mathbb{D}$ was considered in 9 . However, in that paper, a rougher version of the combined estimate was proved under additional smoothness requirements on the data along the edge, and the results obtained in [9] are valid only for edges of opening less than $\pi$.

\section{$\S 1$ Problems in a MOdel CONE AND IN A BOUNDED DOMAIN WITH A CONICAL POINT}

1.1. Preliminaries. Statement of the problem. Let $\mathcal{K}$ be a cone in $\mathbb{R}^{3}$ with vertex at the origin $O$ and such that $\mathcal{K} \cap \mathcal{S}$ is simply connected, and let $G \subset \mathbb{R}^{3}$ be a bounded domain with only one conical point $O$. We assume that $G$ coincides with $\mathcal{K}$ in a neighborhood of $O$ and that the boundaries of $\mathcal{K}$ and $G$ are smooth off $O$. We introduce some function spaces. Suppose $s \in \mathbb{N}$ and $\beta \in \mathbb{R}$. We put $r=\left(x^{2}+y^{2}+z^{2}\right)^{1 / 2}$ and denote by $H_{\beta}^{s}(\mathcal{K})$ the completion of $C_{c}^{\infty}(\overline{\mathcal{K}} \backslash O)$ with respect to the norm

$$
\left\|u ; H_{\beta}^{s}(\mathcal{K})\right\|=\left(\sum_{|\alpha| \leq s} \int_{\mathcal{K}} r^{2(\beta+|\alpha|-s)}\left|D_{x, y, z}^{\alpha} u(x, y, z)\right|^{2} d x d y d z\right)^{1 / 2}
$$

where $\alpha=\left(\alpha_{1}, \alpha_{2}, \alpha_{3}\right)$ is a multiindex, $D_{x, y, z}^{\alpha}=D_{x}^{\alpha_{1}} D_{y}^{\alpha_{2}} D_{z}^{\alpha_{3}}$, and $D_{x}=-i \partial / \partial x$. The space $H_{\beta}^{s}(\mathcal{K}, q)$ with $q>0$ is endowed with the norm

$$
\left\|u ; H_{\beta}^{s}(\mathcal{K}, q)\right\|=\left(\sum_{k=0}^{s} q^{2 k}\left\|u ; H_{\beta}^{s-k}(\mathcal{K})\right\|^{2}\right)^{1 / 2}
$$

Replacing $\mathcal{K}$ by $G$, in a similar way we define the spaces $H_{\beta}^{s}(G)$ and $H_{\beta}^{s}(G, q)$. In the cylinder $\mathcal{Q}=\mathcal{K} \times \mathbb{R}$, we introduce the space $H_{\beta}^{s}(\mathcal{Q})$ obtained by completing the space $C_{c}^{\infty}((\overline{\mathcal{K}} \backslash O) \times \mathbb{R})$ in the norm

$$
\left\|w ; H_{\beta}^{s}(\mathcal{Q})\right\|=\left(\sum_{|\alpha| \leq s} \int_{\mathcal{K}} \int_{\mathbb{R}} r^{2(\beta+|\alpha|-s)}\left|D_{x, y, z, t}^{\alpha} w(x, y, z, t)\right|^{2} d x d y d z d t\right)^{1 / 2} .
$$

The space $H_{\beta}^{s}(\mathcal{Q}, q)$ with $q>0$ is equipped with the norm

$$
\left\|u ; H_{\beta}^{s}(\mathcal{Q}, q)\right\|=\left(\sum_{k=0}^{s} q^{2 k}\left\|u ; H_{\beta}^{s-k}(\mathcal{Q})\right\|^{2}\right)^{1 / 2}
$$

Again, replacing $\mathcal{K}$ by $G$, we define the spaces $H_{\beta}^{s}(\mathbf{Q})$ and $H_{\beta}^{s}(\mathbf{Q}, q)$ in the cylinder $\mathbf{Q}=G \times \mathbb{R}$. Finally, $V_{\beta}^{s}(\mathcal{Q}, \gamma)$ and $V_{\beta}^{s}(\mathbf{Q}, \gamma)$ with $\gamma>0$ denote the spaces with the norms

$$
\left\|w ; V_{\beta}^{s}(\mathcal{Q}, \gamma)\right\|=\left\|w^{\gamma} ; H_{\beta}^{s}(\mathcal{Q}, \gamma)\right\|
$$

and

$$
\left\|w ; V_{\beta}^{s}(\mathbf{Q}, \gamma)\right\|=\left\|w^{\gamma} ; H_{\beta}^{s}(\mathbf{Q}, \gamma)\right\|,
$$

respectively, where $w^{\gamma}=e^{-\gamma t} w$. 
The augmented Maxwell system

$$
\left\{\begin{array}{c}
\partial \vec{u} / \partial t-\operatorname{curl} \vec{v}+\nabla h=\vec{f}_{1}, \\
\partial \vec{v} / \partial t+\operatorname{curl} \vec{u}+\nabla q=\vec{f}_{2}, \\
\partial h / \partial t+\operatorname{div} \vec{u}=g_{1}, \\
\partial q / \partial t+\operatorname{div} \vec{v}=g_{2}
\end{array}\right.
$$

will be considered in $\mathbf{Q}$ (or $\mathcal{Q}$ ) with boundary conditions corresponding to the ideal conductive boundary:

$$
\vec{n} \times \vec{u}=0, \quad(\vec{n} \cdot \vec{v})=0, \quad h=0,
$$

where $\vec{n}$ is the unit outward normal. System (1) will be abbreviated to the form

$$
\partial \mathcal{U} / \partial t+A(\partial) \mathcal{U}=\mathcal{F}
$$

where $\mathcal{U}=(\vec{u}, \vec{v}, h, q)^{T}$ and $\partial=\left(\partial_{x}, \partial_{y}, \partial_{z}\right)$. We formulate some properties of the augmented Maxwell system.

Proposition 1.1.1. 1) The operator $A(\partial)$ is elliptic and is not strongly elliptic.

2) The Green formula is valid:

(4) $\int_{V}\left\langle A(\partial) \mathcal{U}_{1}, \mathcal{U}_{2}\right\rangle d V+\int_{V}\left\langle\mathcal{U}_{1}, A(\partial) \mathcal{U}_{2}\right\rangle d V=\int_{\partial V}\left\langle\Gamma \mathcal{U}_{1}, T \mathcal{U}_{2}\right\rangle d S+\int_{\partial V}\left\langle T \mathcal{U}_{1}, \Gamma \mathcal{U}_{2}\right\rangle d S$, where $V \subset \mathbb{R}^{3}$ is a domain with smooth boundary, $\mathcal{U}_{1}, \mathcal{U}_{2} \in \mathcal{C}^{\infty}(\bar{V})$, and where we put $\Gamma \mathcal{U}=(\vec{n} \times \vec{u},(\vec{n} \cdot \vec{v}), h)^{T}, T \mathcal{U}=(\vec{v}, q,(\vec{n} \cdot \vec{u}))^{T}$.

3) The system $A(\partial) \mathcal{U}=\mathcal{F}$ with boundary conditions $\Gamma \mathcal{U}=\mathcal{H}$ is an elliptic boundary value problem selfadjoint with respect to the Green formula (4).

Let $\tau=\sigma-i \gamma, \sigma \in \mathbb{R}, \gamma>0$. Applying the Fourier transform $\mathcal{F}_{t \rightarrow \tau}$ to problem (1), (2), we obtain a problem with parameter $\tau$ in the cone $\mathcal{K}$ or in the domain $G$ :

$$
\begin{gathered}
\tau \widehat{\mathcal{U}}+A(D) \widehat{\mathcal{U}}=\widehat{\mathcal{F}}, \\
\vec{n} \times \widehat{\vec{u}}=0, \quad(\vec{n} \cdot \widehat{\vec{v}})=0, \quad \widehat{h}=0 .
\end{gathered}
$$

When dealing with the problem in $\mathcal{K}$, we can change the variables $\eta=(|\tau| x,|\tau| y,|\tau| z)$. We denote $\tau /|\tau|$ by $\theta$, put $M\left(D_{\eta}, \theta\right)=\theta+A\left(D_{\eta}\right), U(\eta, \tau)=\widehat{\mathcal{U}}\left(|\tau|^{-1} \eta, \tau\right), F(\eta, \tau)=$ $|\tau|^{-1} \widehat{\mathcal{F}}\left(|\tau|^{-1} \eta, \tau\right)$, and rewrite system (5) in the form

$$
M\left(D_{\eta}, \theta\right) U=F .
$$

1.2. Operator pencil. On the functions $\Phi \in H^{1}(\Xi)$ such that $r^{i \lambda} \Phi(\varphi, \vartheta)$ satisfies (2) on $\partial \mathcal{K}$, we define an operator pencil by the formula

$$
\mathfrak{A}(\lambda) \Phi(\varphi, \vartheta)=r^{1-i \lambda} A\left(D_{x}, D_{y}, D_{z}\right) r^{i \lambda} \Phi(\varphi, \vartheta) .
$$

Here $(r, \vartheta, \varphi)$ are the spherical coordinates centered at $O$ and $\Xi=\mathcal{K} \cap S^{2}$. We write the boundary conditions for $\Phi$ in an explicit form. Let $\left(\vec{e}_{r}, \vec{e}_{\vartheta}, \vec{e}_{\varphi}\right)$ be unit basis vectors in a spherical coordinate system, let $\vec{\sigma}$ be a vector tangent to $\partial \Xi$, and let $\vec{\Phi}=(\vec{U}, \vec{V}, H, Q)$. Then the boundary conditions on $\partial \Xi$ take the form

$$
\left(\vec{e}_{r} \cdot \vec{U}\right)=0, \quad(\vec{\sigma} \cdot \vec{U})=0, \quad(\vec{n} \cdot \vec{V})=0, \quad H=0,
$$

where $\vec{n}$ denotes the unit outward normal to the boundary of $\mathcal{K}$. Since $\mathfrak{A}$ is an elliptic pencil, its spectrum consists of normal eigenvalues $\left\{\lambda_{k}\right\}_{k \in \mathbb{N}}$.

Proposition 1.2.1. All eigenvalues of $\mathfrak{A}$ lie on the imaginary axis, and a finite collection of linearly independent eigenvectors $\Phi_{s, j}, s=1, \ldots, N_{j}$, corresponds to every eigenvalue $\lambda_{j}$. There are no root vectors. 
Proof. In the spherical coordinates, the operator $A(D)$ takes the form

$$
A(D)=A_{1}(\varphi, \vartheta) D_{r}+(1 / r) A_{2}(\varphi, \vartheta) D_{\vartheta}+(1 / r) A_{3}(\varphi, \vartheta) D_{\varphi},
$$

where the $A_{i}(\varphi, \vartheta)$ are $(8 \times 8)$-matrices that can easily be calculated. These matrices satisfy

$$
\begin{gathered}
A_{1} \cdot A_{1}=I, \quad A_{2} \cdot A_{2}=I, \quad \sin ^{2} \vartheta \cdot A_{3} \cdot A_{3}=I, \\
A_{1} \cdot A_{2}+A_{2} \cdot A_{1}=0, \quad \sin \vartheta\left(A_{1} \cdot A_{3}+A_{3} \cdot A_{1}\right)=0, \\
\partial A_{1} / \partial \vartheta=A_{2}, \quad \partial A_{2} / \partial \vartheta=-A_{1} .
\end{gathered}
$$

From (9) it follows that

$$
\mathfrak{A}(\lambda)=\lambda A_{1}+A_{2} D_{\vartheta}+A_{3} D_{\varphi} .
$$

Let $\lambda_{m}$ be an eigenvalue of the operator pencil and $\Phi_{m}$ an eigenvector, i.e., $\mathfrak{A}\left(\lambda_{m}\right) \Phi_{m}=0$. Then $B \Phi_{m}=\lambda_{m} \Phi_{m}$, where $B$ is the operator defined by the formula $B=-A_{1} \cdot A_{2} D_{\vartheta}-$ $A_{1} \cdot A_{3} D_{\varphi}$ on the domain of $\mathfrak{A}$. Using the Green formula (4), it is not hard to verify that the operator $i B$ is symmetric on $L_{2}(\Xi)$. Since the problem $\{A(D), \Gamma\}$ is elliptic (see Proposition 1.1.1), the operator $i B$ with such a domain is selfadjoint. Since its domain is compactly embedded in $L_{2}(\Xi)$, the spectrum of $B$ is discrete. Obviously, the spectrum of $B$ coincides with that of the pencil $\mathfrak{A}$. Therefore, the eigenvalues of the pencil lie on the imaginary axis. We show that there are no root vectors. Let $\lambda_{m}$ and $\Phi_{m}$ be an eigenvalue and an eigenvector of $\mathfrak{A}$, and let $\widetilde{\Phi}$ be a root vector. In other words, $\mathfrak{A}\left(\lambda_{m}\right) \widetilde{\Phi}=\partial_{\lambda} \mathfrak{A}\left(\lambda_{m}\right) \Phi_{m}$ or, equivalently, $\left(B-\lambda_{m}\right) \widetilde{\Phi}=\Phi_{m}$. However, $\Phi_{m}$ is not orthogonal to the subspace $\operatorname{ker}\left(B-\lambda_{m}\right)$. Therefore, $\widetilde{\Phi}$ does not exist.

Consider the pencil $\mathfrak{A}^{*}(\lambda)$ defined by $\mathfrak{A}^{*}(\lambda)=(\mathfrak{A}(\bar{\lambda}))^{*}$. It is known that if $\mu$ is an eigenvalue of $\mathfrak{A}$, then $\bar{\mu}$ is an eigenvalue of $\mathfrak{A}^{*}$, and their multiplicities coincide. The corresponding eigenfunctions $\left\{\Phi_{s}\right\}_{s=1, \ldots, N}$ and $\left\{\Psi_{s}\right\}_{s=1, \ldots, N}$ can be chosen so as to satisfy the orthogonality and normalization conditions

$$
\int_{\Xi}\left\langle\partial_{\mu} \mathfrak{A}(\mu) \Phi_{s}, \Psi_{p}\right\rangle \sin \vartheta d \vartheta d \varphi=\delta_{s, p}
$$

(see [11] or [12]). Using (11) and integrating by parts, we show easily that $\mathfrak{A}^{*}(\lambda)=$ $\mathfrak{A}(\lambda+2 i)$. Therefore, the above results can be reformulated as follows. If $\mu$ is an eigenvalue of $\mathfrak{A}$, then so is $\bar{\mu}+2 i$, the multiplicities of these eigenvalues coincide, and the eigenfunctions can be chosen so as to satisfy the orthogonality and normalization conditions (12). The numbers $\mu$ and $\bar{\mu}+2 i$ are symmetric with respect to the point $i$. Formula (11) and the explicit form of $A_{1}(\varphi, \vartheta)$ allow us to check that if $\Phi$ is an eigenvector of the pencil $\mathfrak{A}$ corresponding to an eigenvalue $\lambda$, then $A_{1} \Phi$ also is an eigenvector of $\mathfrak{A}$ and corresponds to the eigenvalue $\bar{\lambda}+2 i$. The pencil has a block structure. One block acts on the components $(\vec{U}, Q)$ and the other on the components $(\vec{V}, H)$ of the function $\Phi=(\vec{U}, \vec{V}, H, Q)$. The matrix $A_{1}$ acts on the components by the rule

$$
\begin{aligned}
& A_{1}(\vec{U}, \overrightarrow{0}, 0, Q)=\left(\overrightarrow{0}, \overrightarrow{e_{r}} \times \vec{U}+Q \vec{e}_{r},\left(\vec{e}_{r} \cdot \vec{U}\right), 0\right)^{T}, \\
& A_{1}(\overrightarrow{0}, \vec{V}, H, 0)=\left(-\vec{e}_{r} \times \vec{V}+H \vec{e}_{r}, \overrightarrow{0}, 0,\left(\vec{e}_{r} \cdot \vec{V}\right)\right)^{T} .
\end{aligned}
$$

We recall that the eigenvectors of the pencil are also eigenvectors of some selfadjoint operator (see Proposition 1.2.1). Therefore, the eigenvectors corresponding to different eigenvalues are orthogonal. This fact, the block structure of $\mathfrak{A}$, and the relation $\partial_{\lambda} \mathfrak{A}(\lambda)=$ $A_{1}$ allow us to choose the eigenvectors of the pencil in the form $(\vec{U}, \overrightarrow{0}, 0, Q)$ or $(\overrightarrow{0}, \vec{V}, H, 0)$ and satisfying the orthogonality and normalization conditions (12). In the following three lemmas, we describe the properties of eigenvalues and eigenvectors of the pencil in more detail. 
Lemma 1.2.2. If the strip $\{\lambda \in \mathbb{C}: \operatorname{Im} \lambda \in] 0,1[\}$ contains some eigenvalues of $\mathfrak{A}$, then the components $H$ and $Q$ of the corresponding eigenvectors vanish.

Proof. Let $\Phi=(\vec{U}, \overrightarrow{0}, 0, Q)$, and let $(\Phi, \lambda)$ be an eigenvector and an eigenvalue of the pencil $\mathfrak{A}$. Then $A\left(D_{x}, D_{y}, D_{z}\right) r^{i \lambda} \Phi=0$. Applying the operator $A\left(D_{x}, D_{y}, D_{z}\right)$ once again, we obtain $\Delta r^{i \lambda} \Phi=0$. We find the boundary conditions satisfied by $Q$. Since $r^{i \lambda} \vec{U} \times \vec{n}=0$, we have $\left(\operatorname{curl}\left(r^{i \lambda} \vec{U}\right) \cdot \vec{n}\right)=0$. The relation $\operatorname{curl}\left(r^{i \lambda} \vec{U}\right)+\nabla\left(r^{i \lambda} Q\right)=0$ implies that $\partial\left(r^{i \lambda} Q\right) / \partial n=0$ on $\partial \mathcal{K}$. This means that the pair $(\lambda, Q)$ consists of an eigenvalue and an eigenvector of the pencil corresponding to the Neumann problem for the Laplace operator. Similarly, if $\Phi=(\overrightarrow{0}, \vec{V}, H, 0)$, then $(\lambda, H)$ consists of an eigenvalue and an eigenvector of the pencil corresponding to the Dirichlet problem for the Laplace operator. However, for a cone $\mathcal{K} \subset \mathbb{R}^{3}$ the strip $\{\lambda \in \mathbb{C}: \operatorname{Im} \lambda \in] 0,1[\}$ contains no eigenvalues of these pencils (see [1, §3] and [4, §1]).

Lemma 1.2.3. Let $\lambda$ be an eigenvalue of the pencil $\mathfrak{A}, \operatorname{Im} \lambda \in] 1,2[$, and let $\Phi$ be an eigenvector corresponding to $\lambda$. If $\Phi=(\vec{U}, \overrightarrow{0}, 0, Q)$, then $Q \not \equiv 0$, and if $\Phi=(\overrightarrow{0}, \vec{V}, H, 0)$, then $H \not \equiv 0$.

Proof. We consider one of these cases; the other can be treated in a similar way. Assume that $\Phi=(\vec{U}, \overrightarrow{0}, 0, Q)$. Then $\Psi=A_{1} \Phi$ is an eigenvector of $\mathfrak{A}$ corresponding to the eigenvalue $\mu=\bar{\lambda}+2 i$. Moreover, $\operatorname{Im} \mu \in] 0,1\left[\right.$ and $\Psi=\left(\overrightarrow{0}, \vec{e}_{r} \times \vec{U}+Q \vec{e}_{r},\left(\vec{e}_{r} \cdot \vec{U}\right), 0\right)$. By Lemma 1.2.2, we have $\left(\vec{e}_{r} \cdot \vec{U}\right)=0$. Suppose $Q=0$. We rewrite the pencil $\operatorname{curl}\left(r^{i \lambda} \vec{U}\right)+$ $\nabla\left(r^{i \lambda} Q\right)=0, \operatorname{div}\left(r^{i \lambda} \vec{U}\right)=0$ in the spherical coordinates:

$$
\left\{\begin{array}{c}
\frac{\cos \vartheta}{\sin \vartheta} U_{\varphi}+\partial_{\vartheta} U_{\varphi}-\frac{1}{\sin \vartheta} \partial_{\varphi} U_{\vartheta}+i \lambda Q=0, \\
-(i \lambda+1) U_{\varphi}+\frac{1}{\sin \vartheta} \partial_{\varphi} U_{r}+\partial_{\vartheta} Q=0, \\
(i \lambda+1) U_{\vartheta}+\frac{1}{\sin \vartheta} \partial_{\varphi} Q-\partial_{\vartheta} U_{r}=0, \\
(2+i \lambda) U_{r}+\frac{\cos \vartheta}{\sin \vartheta} U_{\vartheta}+\partial_{\vartheta} U_{\vartheta}+\frac{1}{\sin \vartheta} \partial_{\varphi} U_{\varphi}=0,
\end{array}\right.
$$

where $\vec{U}=U_{r} \vec{e}_{r}+U_{\vartheta} \vec{e}_{\vartheta}+U_{\varphi} \vec{e}_{\varphi}$. Since $U_{r}=Q=0$ and $\lambda \neq i$, we obtain $U_{\varphi}=U_{\vartheta}=0$, whence $\Phi=0$. This contradiction completes the proof.

Lemma 1.2.4. The point $\lambda=i$ is regular for the pencil $\mathfrak{A}$.

Proof. Suppose that $\lambda=i$ is an eigenvalue of $\mathfrak{A}$. Let $\Phi=(\vec{U}, \overrightarrow{0}, 0, Q)$ be an eigenvector corresponding to this eigenvalue. Then $\Psi=A_{1} \Phi=\left(\overrightarrow{0},-\vec{U} \times \vec{e}_{r}+Q \vec{e}_{r},\left(\vec{U} \cdot \vec{e}_{r}\right), 0\right)$ is also an eigenvector for $\lambda=i$. Arguing as in the proof of Lemma 1.2.2, we see that $Q=$ const and $H=\left(\vec{e}_{r} \cdot \vec{U}\right)=0$. Indeed, $\lambda=i$ is a regular point for the operator pencil of the Dirichlet problem for the Laplace operator. In the case of the Neumann problem, the eigenvector for $\lambda=i$ is a constant. Then for $\vec{V}=-\vec{U} \times \vec{e}_{r}+Q \vec{e}_{r}$ we have $\operatorname{curl}\left(r^{-1} \vec{V}\right)=0$ and $\operatorname{div}\left(r^{-1} \vec{V}\right)=0$. Since the cone $\mathcal{K}$ is simply connected, this implies that $r^{-1} \vec{V}=\nabla Z$, where $\Delta Z=0, \partial Z / \partial n=0$. We rewrite the relation $r^{-1} \vec{V}=\nabla Z$ in the spherical coordinates: $r^{-1} \vec{V}=(\partial Z / \partial r) \vec{e}_{r}+r^{-1}(\partial Z / \partial \vartheta) \vec{e}_{\vartheta}+(r \sin \vartheta)^{-1}(\partial Z / \partial \varphi) \vec{e}_{\varphi}$, obtaining $Z=Q \log r+A(\varphi, \vartheta)$. The function $Z$ is a solution of the homogeneous Neumann problem for the Laplace operator in $\mathcal{K} \subset \mathbb{R}^{3}$. The results on the asymptotics of solutions of elliptic problems near singularities of the boundary (see [12]) imply that $Q=0, A(\varphi, \vartheta)=$ const. It follows that $\vec{V}=0$ and $\vec{U}=0$.

Let $\lambda_{k}$ with $k>0$ denote the eigenvalues of $\mathfrak{A}$ with imaginary part greater than 1 , $\operatorname{Im} \lambda_{k} \leq \operatorname{Im} \lambda_{k+1}$, and let $\lambda_{-k}$ with $k>0$ be the eigenvalues of the pencil that are 
symmetric to $\lambda_{k}$ with respect to the point $\lambda=i$. Let $\left\{\Phi_{s, k}\right\}_{s=1, \ldots, N_{k}}$ be a basis in the eigenspace corresponding to $\lambda_{k}$. Some of the properties of the pencil $\mathfrak{A}$ are listed in the following proposition.

Proposition 1.2.5. The eigenvectors $\left\{\Phi_{s, k}\right\}_{s=1, \ldots, N_{k}}$ corresponding to the eigenvalues $\lambda_{k}$ of the pencil $\mathfrak{A}$ can be taken in the form $(\vec{U}, \overrightarrow{0}, 0, Q)$ or $(\overrightarrow{0}, \vec{V}, H, 0)$. Moreover,

$$
\int_{\Xi}\left\langle\partial_{\lambda} \mathfrak{A}\left(\lambda_{k}\right) \Phi_{s, k}, \Phi_{m, p}\right\rangle \sin \vartheta d \vartheta d \varphi=\delta_{k,-p} \cdot \delta_{s, m},
$$

where $k, p=\mp 1, \mp 2, \ldots$, and $\delta_{k, p}$ is the Kronecker symbol.

We introduce the notation

$$
u_{s, k}=r^{i \lambda_{k}} \Phi_{s, k} .
$$

For the functions $u_{s, k}$ we have $A(D) u_{s, k}=0$ and $\Gamma u_{s, k}=0$ in $\mathcal{K}$.

\subsection{A global energy estimate.}

Proposition 1.3.1. Suppose $v=(\vec{u}, \vec{v}, h, q)^{T}$ is a vector-valued function that belongs to $\mathcal{C}_{c}^{\infty}\left(\bar{G} \backslash O, \mathbb{C}^{8}\right)$ and satisfies the boundary conditions (2). Then

$$
\gamma\left\|v ; L_{2}(G)\right\| \leq\left\|M\left(D_{x}, D_{y}, D_{z}, \tau\right) v ; L_{2}(G)\right\|,
$$

where $M\left(D_{x}, D_{y}, D_{z}, \tau\right) v=\tau v+A\left(D_{x}, D_{y}, D_{z}\right) v$.

Proof. Let $u(x, y, z, t)=\psi(t) v(x, y, z)$, where $\psi(t) \in \mathcal{C}_{0}^{\infty}(\mathbb{R})$. Using the Green formula (4) and the boundary conditions, we obtain

$$
\operatorname{Re} \int_{G}\langle A(\partial) u(x, y, z, t), u(x, y, z, t)\rangle d x d y d z=0,
$$

where $\langle$,$\rangle denotes the inner product on \mathbb{C}^{8}$. Then

$$
\frac{d}{d t}\left\|u ; L_{2}(G)\right\|^{2}=2 \operatorname{Re} \int_{G}\left\langle u, u_{t}+A(\partial) u\right\rangle d x d y d z .
$$

Therefore, we have

$$
\frac{d}{d t}\left\|u(\cdot, t) ; L_{2}(G)\right\|^{2} \leq\left\|u ; L_{2}(G)\right\| \cdot\left\|M\left(D_{x}, D_{y}, D_{z}, D_{t}\right) u ; L_{2}(G)\right\|,
$$

whence

$$
\left\|u(\cdot, t) ; L_{2}(G)\right\|^{2} \leq 2 \int_{-\infty}^{t}\left\|u(\cdot, s) ; L_{2}(G)\right\| \cdot\left\|M\left(D_{x}, D_{y}, D_{z}, D_{s}\right) u(\cdot, s) ; L_{2}(G)\right\| d s .
$$

We multiply by $e^{-2 \gamma t}$ and integrate from $-\infty$ to $+\infty$. Changing the order of integration on the right-hand side, we obtain

$$
\begin{aligned}
& \int_{-\infty}^{+\infty} e^{-2 \gamma t}\left\|u(\cdot, t) ; L_{2}(G)\right\|^{2} d t \\
& \quad \leq \gamma^{-1} \int_{-\infty}^{+\infty} e^{-2 \gamma t}\left\|u(\cdot, t) ; L_{2}(G)\right\|\left\|M\left(D_{x}, D_{y}, D_{z}, D_{t}\right) u(\cdot, t) ; L_{2}(G)\right\| d t .
\end{aligned}
$$

Application of the Cauchy inequality to the right-hand side yields

$$
\gamma^{2} \int_{-\infty}^{+\infty} e^{-2 \gamma t}\left\|u(\cdot, t) ; L_{2}(G)\right\|^{2} d t \leq \int_{-\infty}^{+\infty} e^{-2 \gamma t}\left\|M\left(D_{x}, D_{y}, D_{z}, D_{t}\right) u(\cdot, t) ; L_{2}(G)\right\|^{2} d t .
$$


By Parseval's identity,

$$
\begin{aligned}
& \gamma^{2} \int_{G} d x d y d z \int_{\mathbb{R}-i \gamma} d \tau|\hat{\psi}(\tau)|^{2}|v(x, y, z)|^{2} \\
& \quad \leq \int_{G} d x d y d z \int_{\mathbb{R}-i \gamma} d \tau|\hat{\psi}(\tau)|^{2}\left|M\left(D_{x}, D_{y}, D_{z}, \tau\right) v(x, y, z)\right|^{2} .
\end{aligned}
$$

Since $\psi$ is an arbitrary function in $\mathcal{C}_{0}^{\infty}(\mathbb{R})$, this leads to the required estimate (15).

Remark 1.3.2. The same estimate is valid in the cone $\mathcal{K}$ for the functions of class $\mathcal{C}_{c}^{\infty}(\overline{\mathcal{K}} \backslash O)$ subject to the boundary conditions (2).

Remark 1.3.3. Inequality (15) remains true if we replace $\tau$ with $\bar{\tau}$.

In what follows it is assumed tacitly that $v$ is a function in $\mathcal{C}_{c}^{\infty}\left(\bar{G} \backslash O, \mathbb{C}^{8}\right), v=$ $(\vec{u}, \vec{v}, h, q)^{T}$, and $v$ satisfies the boundary conditions (2). As a rule, we only mention that a function belongs to $\mathcal{C}_{c}^{\infty}(\bar{G} \backslash O)$ (or some other function class) and is subject to (2).

Our next goal is to extend (15) to the linear set consisting of all linear combinations of the form $u=v+\chi \sum_{k, s} u_{s, k}$. Here $v$ is in $\mathcal{C}_{c}^{\infty}(\bar{G} \backslash O)$ and satisfies (2). By $\chi$ we denote a cut-off function that is equal to 1 near the point $O$ and vanishes outside a neighborhood in which $G$ coincides with $\mathcal{K}$. The functions $u_{s, k}$ are defined by (14). Since we require that $\chi u_{s, k} \in L_{2}(G)$, the above linear set contains only $\chi u_{s, k}$ with $\operatorname{Im} \lambda_{k}<3 / 2$. The right-hand side $M\left(D_{x}, D_{y}, D_{z}, \tau\right) \chi u_{s, k}$ belongs to $L_{2}(G)$ because $A\left(D_{x}, D_{y}, D_{z}\right) u_{s, k}=0$ (see Subsection 1.2). Moreover, when proving (15) on $D(G)$, we assume that

$$
\operatorname{Re} \int_{G}\langle A(\partial) u, u\rangle d x d y d z=0 .
$$

If there is an eigenvalue $\lambda_{k}$ of the pencil $\mathfrak{A}$ such that $\operatorname{Im} \lambda_{k} \in[1,3 / 2$ [, then, by (13), we have

$$
\operatorname{Re} \int_{G}\langle A(\partial) w, w\rangle d x d y d z=2 \operatorname{Re}(\alpha \bar{\beta})
$$

for $w=\chi\left(\alpha u_{s, k}+\beta u_{s,-k}\right)$. Therefore, for all $\lambda_{k}$ in the strip $\left.\operatorname{Im} \lambda \in\right] 1 / 2,3 / 2[$, in the linear set in question we include combinations of the form $\chi\left(\alpha_{s, k} u_{s, k}+\beta_{s, k} u_{s,-k}\right)$ where $\alpha_{s, k}, \beta_{s, k}$ are some fixed coefficients satisfying $\operatorname{Re} \alpha_{s, k} \overline{\beta_{s, k}}=0,\left|\alpha_{s, k}\right|+\left|\beta_{s, k}\right|>0$. The meaning of the latter condition will be explained later. It is easy to see that the proof of (15) remains valid for the functions in the modified linear set. In the definition and proposition that follow we summarize the results obtained.

Definition 1.3.4. We denote by $D(G)$ the linear set spanned by the functions of the following 3 types: the $\mathcal{C}_{c}^{\infty}(\bar{G} \backslash O)$-functions satisfying the boundary conditions (2) on $\partial G$; the functions $\chi u_{s, k}$ for $\operatorname{Im} \lambda_{k} \leq 1 / 2$; the functions $\chi\left(\alpha_{s, k} u_{s, k}+\beta_{s, k} u_{s,-k}\right)$ for $\operatorname{Im} \lambda_{k} \in$ ] $1,3 / 2\left[\right.$, where $\alpha_{s, k}, \beta_{s, k}$ are some fixed coefficients such that $\operatorname{Re} \alpha_{s, k} \overline{\beta_{s, k}}=0,\left|\alpha_{s, k}\right|+$ $\left|\beta_{s, k}\right|>0$. The linear set $D(\mathcal{K})$ is defined in the same way with $G$ replaced by $\mathcal{K}$.

Proposition 1.3.5. Estimate (15) is true for any function in $D(G)$.

In what follows we consider only the problem in $G$. However, all results remain valid for the problem in $\mathcal{K}$. At the end of the section we formulate the corresponding statements.

With problem (5), (6) we associate the unbounded operator

$$
v \mapsto M(\tau) v:=M\left(D_{x}, D_{y}, D_{z}, \tau\right) v
$$

on $L_{2}(G)$. As the domain $\mathcal{D} M(\tau)$, we take $D(G)$ (see Definition 1.3.4). The operator $M(\tau)$ admits closure. Indeed, let $\left\{v_{k}\right\} \subset \mathcal{D} M(\tau)$ be such that $v_{k} \rightarrow 0$ and $M(\tau) v_{k} \rightarrow f$ in $L_{2}(G)$. Then $\left(M(\tau) v_{k}, w\right)=\left(v_{k}, M(\bar{\tau}) w\right)$ for any $w$ in $\mathcal{C}_{c}^{\infty}(G)$. Letting $k \rightarrow+\infty$, we 
obtain $(f, w)=0$, whence $f=0$. In what follows we consider the closed operator only, keeping the notation $M(\tau)$ and $\mathcal{D} M(\tau)$ for the operator and its domain. Clearly, we have

$$
\gamma\left\|v ; L_{2}(G)\right\| \leq\left\|M(\tau) v ; L_{2}(G)\right\|
$$

for any $v \in \mathcal{D} M(\tau)$. The next statement follows from (16).

Proposition 1.3.6. Ker $M(\tau)=0$, and the range $\operatorname{Im} M(\tau)$ of $M(\tau)$ is closed in $L_{2}(G)$.

Proposition 1.3.7. $\operatorname{Im} M(\tau)=L_{2}(G)$.

Proof. It suffices to check that $\operatorname{Ker} M(\tau)^{*}=\{0\}$. Suppose $w \in \operatorname{Ker} M(\tau)^{*}$. Then, by the local properties of solutions of elliptic problems (see [12]), we have $w \in \mathcal{C}^{\infty}(\bar{G} \backslash O)$, and $w$ satisfies the homogeneous problem that is formally adjoint with respect to the Green formula (4):

$$
\begin{array}{rlrl}
M\left(D_{x}, D_{y}, D_{z}, \bar{\tau}\right) w & =0, \quad(x, y, z) & \in G, \\
\Gamma w & =0, \quad(x, y, z) \in \partial G \backslash O .
\end{array}
$$

In a neighborhood of $O$, the function $w$ admits an asymptotic representation of the form

$$
w \sim \chi \sum_{k} \sum_{s=1, \ldots, N_{k}} c_{s, k} V_{s, k, T} .
$$

Here $\chi$ stands for a cut-off function equal to 1 near $O$, and $V_{s, k, T}$ is the sum of the first $T$ terms of the formal series

$$
V_{s, k}(x, y, z, \bar{\tau})=r^{i \lambda_{-k}} \sum_{q=0}^{\infty} r^{q} \bar{\tau}^{q} \Psi_{q}(\vartheta, \varphi)
$$

(where $\Psi_{0}=\Phi_{s,-k}$ ), which satisfies (17) and (18); for more details, we refer the reader, e.g., to [2] or [12]. Since $w \in \mathcal{D} M(\tau)^{*} \subset L_{2}(G)$, the asymptotics of $w$ may contain only the sums $V_{s, k, T}$ such that $\chi V_{s, k, T} \in L_{2}(G)$. Moreover, there are other restrictions on the terms of the above asymptotics. We find out what functions $\chi u_{s, k}$ are actually in the domain of $M(\tau)^{*}$. It is easily seen that $\chi u_{s, k} \in \mathcal{D} M(\tau)^{*}$ for $\lambda_{k}$ with $\operatorname{Im} \lambda_{k} \leq 1 / 2$. Consider the eigenvalues of the pencil $\mathfrak{A}$ in the strip $\operatorname{Im} \lambda \in] 1 / 2,3 / 2[$. The domain $\mathcal{D} M(\tau)$ contains terms of the form $\chi\left(\alpha_{s, k} u_{s, k}+\beta_{s, k} u_{s,-k}\right)$ with $\alpha_{s, k}, \beta_{s, k}$ satisfying $\operatorname{Re} \alpha_{s, k} \overline{\beta_{s, k}}=0,\left|\alpha_{s, k}\right|+\left|\beta_{s, k}\right|>0$. By (13),

$$
\begin{aligned}
& \left(M\left(D_{x}, D_{y}, D_{z}, \tau\right) \chi\left(\alpha_{s, k} u_{s, k}+\beta_{s, k} u_{s,-k}\right), \chi\left(c u_{s, k}+d u_{s,-k}\right)\right)_{G} \\
& \quad=(1 / i)\left(\alpha_{s, k} \bar{d}+\beta_{s, k} \bar{c}\right) \\
& \quad+\left(\chi\left(\alpha_{s, k} u_{s, k}+\beta_{s, k} u_{s,-k}\right), M\left(D_{x}, D_{y}, D_{z}, \bar{\tau}\right) \chi\left(c u_{s, k}+d u_{s,-k}\right)\right)_{G} .
\end{aligned}
$$

The functions $\chi\left(c u_{s, k}+d u_{s,-k}\right)$ will belong to $\mathcal{D} M(\tau)^{*}$ if we require that $\alpha_{s, k} \bar{d}+\beta_{s, k} \bar{c}=0$. Together with the relation $\operatorname{Re} \alpha_{s, k} \overline{\beta_{s, k}}=0$, this leads to the identity

$$
\chi\left(c u_{s, k}+d u_{s,-k}\right)=\frac{c}{\alpha_{s, k}} \chi\left(\alpha_{s, k} u_{s, k}+\beta_{s, k} u_{s,-k}\right)
$$

for $\alpha_{s, k} \neq 0$. On the other hand, if $\alpha_{s, k}=0$, then $\beta_{s, k} \neq 0$. Then, since $\beta_{s, k} \bar{c}=0$, we obtain $c=0$. Thus, the domain $\mathcal{D} M(\tau)^{*}$ admits the same combinations of the functions $\chi u_{s, k}$ as $D(G)$. Therefore, the functions $V_{s, k, T}$ corresponding to the eigenvalues of $\mathfrak{A}$ in the strip $\operatorname{Im} \lambda \in] 1 / 2,3 / 2[$ are involved in the asymptotics of $w$ in the form of the combinations $\alpha_{s, k} V_{s,-k, T}+\beta_{s, k} V_{s, k, T}$. Now, in order to check that $w=0$, it remains to refer to Remark 1.3.3 and Proposition 1.3.5. 
We discuss the results obtained. We have proved that the operator $M(\tau)$ with domain $D(G)$ admits closure; here $\tau=\sigma-i \gamma, \sigma \in \mathbb{R}, \gamma>0$. The kernel of the closed operator is trivial, and the range of this operator coincides with $L_{2}(G)$. The inverse operator is bounded by (15). The same is true for $M(\bar{\tau})$. The operator $A\left(D_{x}, D_{y}, D_{z}\right)$ with domain $D(G)$ is symmetric. It follows that, for the closed operator $A=\overline{A(D)}$, the operator $(A-\lambda)^{-1}$ exists for all $\lambda \in \mathbb{C} \backslash \mathbb{R}$, and $A$ is selfadjoint. Observe that for the linear set $D(G)$ we can choose various collections $\left\{\alpha_{s, k}, \beta_{s, k}\right\}$ satisfying $\operatorname{Re} \alpha_{s, k} \overline{\beta_{s, k}}=0$ and $\left|\alpha_{s, k}\right|+\left|\beta_{s, k}\right|>0$. This gives rise to various selfadjoint extensions $A$ of $A(D)$. In what follows, unless otherwise stated, as $A$ we take any of these extensions.

Definition 1.3.8. A solution of the equation $(\tau+A) u=f$ with $f \in L_{2}(G)$ is called a strong solution of problem (5), (6).

The next statement summarizes the results of this section.

Theorem 1.3.9. For any $f \in L_{2}(G)$ and any $\tau=\sigma-i \gamma(\sigma \in \mathbb{R}, \gamma>0)$, problem (5), (6) with right-hand side $f$ admits a unique strong solution $v$. This solution satisfies

$$
\gamma\left\|v ; L_{2}(G)\right\| \leq\left\|f ; L_{2}(G)\right\| .
$$

Remark 1.3.10. Theorem[1.3.9 remains true for problem (5), (6) in $\mathcal{K}$.

Remark 1.3.11. Theorem 1.3.9 is valid for problem (5), (6) in $\mathcal{K}$ and in $G$ with $\tau$ replaced by $\bar{\tau}$.

To complete the subsection, we discuss the condition $\left|\alpha_{s, k}\right|+\left|\beta_{s, k}\right|>0$ (see Definition 1.3.4). Assume that $\left|\alpha_{s_{0}, k_{0}}\right|+\left|\beta_{s_{0}, k_{0}}\right|=0$ for some $s_{0}, k_{0}$. Clearly, 15 is true for functions in such a linear set, which will be denoted by $D_{1}(G)$. However, the range of the corresponding closed operator does not coincide with $L_{2}(G)$. The point is that $\mathcal{D} M(\tau)^{*}$ contains linear combinations $\chi\left(\alpha u_{s_{0}, k_{0}}+\beta u_{s_{0},-k_{0}}\right)$ with arbitrary coefficients $\alpha, \beta$, which may fail to satisfy $\operatorname{Re} \alpha \bar{\beta}=0$. Therefore, (15) cannot be applied to $w$ in the kernel of $M(\tau)^{*}$ in order to prove that $w=0$ (see the proof of Proposition 1.3.7). In this case, the kernel of $M(\tau)^{*}$ is of dimension 1 . We construct an element of that kernel. Let $f=M\left(D_{x}, D_{y}, D_{z}, \bar{\tau}\right) \chi u_{s_{0}, k_{0}}$. We need the closure $M_{0}(\bar{\tau})$ of the operator $M\left(D_{x}, D_{y}, D_{z}, \bar{\tau}\right)$ with domain $D_{0}(G)$, where $\alpha_{s_{0}, k_{0}}=0, \beta_{s_{0}, k_{0}}=1$, and the other $\alpha_{s, k}$ and $\beta_{s, k}$ are the same as in $D_{1}(G)$. Let $v$ be a solution of the equation $M_{0}(\bar{\tau}) v=f$. Obviously, the element $w=v-\chi u_{s_{0}, k_{0}}$ belongs to the kernel of $M(\tau)^{*}$. We show that any element in the kernel differs from $w$ by a constant factor. Let $\widetilde{w} \in \operatorname{Ker} M(\tau)^{*}, \widetilde{w} \neq w$. The asymptotics of $\widetilde{w}$ near $O$ involves the term $\chi\left(c u_{s_{0}, k_{0}}+d u_{s_{0},-k_{0}}\right)$ with some $c$ and $d$. Then $\widetilde{\widetilde{w}}=\widetilde{w}+c w$ is in $\mathcal{D} M_{0}(\bar{\tau})$. Since $M_{0}(\bar{\tau}) \widetilde{\widetilde{w}}=0$, we obtain $\widetilde{\widetilde{w}}=0$.

1.4. A combined weighted estimate. In this subsection, we prove a more informative estimate for the solutions of (5), (6) in a bounded domain and in a cone; this estimate will be used in the study of the asymptotics of solutions near the point $O$.

Definition 1.4.1. Let $D_{\beta}(G)$ with $\beta \leq 1$ denote the linear set spanned by the functions of the following three types:

1) the functions in $\mathcal{C}_{c}^{\infty}(\bar{G} \backslash O)$ satisfying the boundary conditions (2) on $\partial G \backslash O$;

2) the functions $\chi u_{s,-k}$ with $k>0$ for the eigenvalues $\lambda_{-k}$ of the pencil $\mathfrak{A}$ such that $\operatorname{Im} \lambda_{-k}<\beta+1 / 2$ and $\operatorname{Im} \lambda_{k} \geq \beta+1 / 2$;

$3)$ the linear combinations of the form $\chi\left(\alpha_{s, k} u_{s, k}+\beta_{s, k} u_{s,-k}\right)$ for the eigenvalues $\lambda_{k}$ $(k>0)$ such that $\operatorname{Im} \lambda_{\mp k}<\beta+1 / 2$, where $\operatorname{Re} \alpha_{s, k} \overline{\beta_{s, k}}=0,\left|\alpha_{s, k}\right|+\left|\beta_{s, k}\right|>0$.

The linear set $D_{\beta}(\mathcal{K})$ is introduced in a similar way. 
Proposition 1.4.2. Let $\beta \leq 1$. Suppose that the line $\operatorname{Im} \lambda=\beta+1 / 2$ contains no points of the spectrum of $\mathfrak{A}$. Then for $v \in D_{\beta}(\mathcal{K})$ we have the inequality

$$
\begin{aligned}
& \gamma^{2}\left\|v ; H_{\beta}^{0}(\mathcal{K})\right\|^{2}+\left\|\chi_{|\tau|} v ; H_{\beta}^{1}(\mathcal{K},|\tau|)\right\|^{2} \\
& \quad \leq c\left\{\left\|f ; H_{\beta}^{0}(\mathcal{K})\right\|^{2}+\left(|\tau|^{1-\beta} / \gamma\right)^{2}\left\|f ; L_{2}(\mathcal{K})\right\|^{2}\right\}
\end{aligned}
$$

where $f=\left(\tau+A\left(D_{x}, D_{y}, D_{z}\right)\right) v, \chi_{|\tau|}(r)=\chi(|\tau| r)$, and $\chi$ is a fixed cut-off function of class $\mathcal{C}_{c}^{\infty}(\overline{\mathcal{K}})$ equal to 1 near the vertex. The constant $c$ is independent of $v$ and $\tau$.

Proof. Step 1. Estimation near the vertex of a cone. We consider the problem (7), (6) in $\mathcal{K}$. In accordance with Proposition 1.1.1, the problem $\left\{A\left(D_{\eta}\right), \Gamma\right\}$ is elliptic. Therefore, if the line $\operatorname{Im} \lambda=\beta+1 / 2$ contains no eigenvalues of the pencil $\mathfrak{A}$, then any function $U \in H_{\beta}^{1}(\mathcal{K}, 1)$ such that $\Gamma U=0$ satisfies

$$
\left\|\chi U ; H_{\beta}^{1}(\mathcal{K})\right\|^{2} \leq c\left\|A\left(D_{\eta}\right) \chi U ; H_{\beta}^{0}(\mathcal{K})\right\|^{2}
$$

(see [12, Chapter 3]). Since $A \chi U=\chi A U+[A, \chi] U$ and $M\left(D_{\eta}, \theta\right)=\theta+A\left(D_{\eta}\right)$, the above inequality can be rewritten as

$$
\left\|\chi U ; H_{\beta}^{1}(\mathcal{K}, 1)\right\|^{2} \leq c\left\{\left\|\chi M\left(D_{\eta}, \theta\right) U ; H_{\beta}^{0}(\mathcal{K})\right\|^{2}+\left\|\psi U ; H_{\beta}^{0}(\mathcal{K})\right\|^{2}\right\},
$$

where $\psi \in \mathcal{C}_{0}^{\infty}(\overline{\mathcal{K}}), \chi \psi=\chi$.

Step 2. Estimation far from the vertex. At this step we prove that the inequality

$$
\begin{aligned}
& (\gamma /|\tau|)^{2}\left\|\kappa_{\infty} U ; H_{\beta}^{0}(\mathcal{K})\right\|^{2} \\
& \quad \leq c\left\{\left\|\kappa_{\infty} M\left(D_{\eta}, \theta\right) U ; H_{\beta}^{0}(\mathcal{K})\right\|^{2}+\left\|\psi_{\infty} U ; H_{\beta-1}^{0}(\mathcal{K})\right\|^{2}\right\},
\end{aligned}
$$

is valid for every $\beta \in \mathbb{R}$ and every $U \in H_{\beta}^{1}(\mathcal{K}, 1)$ satisfying the boundary conditions $\Gamma U=0$. In this inequality, the constant $c$ is independent of $U$ and $\tau$, and $\kappa_{\infty}$ and $\psi_{\infty}$ are smooth functions in $\mathcal{K}$ equal to 0 near the vertex and to 1 in a neighborhood of infinity and satisfying $\kappa_{\infty} \psi_{\infty}=\kappa_{\infty}$.

Suppose $\kappa, \psi \in \mathcal{C}^{\infty}(\overline{\mathcal{K}}), \kappa \psi=\kappa, \operatorname{supp} \kappa \subset\{(x, y, z) \in \mathcal{K}: 1 / 2<r<2\}, \operatorname{supp} \psi \subset$ $\{(x, y, z) \in \mathcal{K}: 1 / 4<r<4\}$. By (15), we have

$$
\gamma^{2}\left\|\kappa U ; L_{2}(\mathcal{K})\right\|^{2} \leq\left\|M\left(D_{x}, D_{y}, D_{z}, \tau\right) \kappa U ; L_{2}(\mathcal{K})\right\|^{2} .
$$

Since $M \kappa U=\kappa M U+[M, \kappa] U$, we obtain

$$
\gamma^{2}\left\|\kappa U ; L_{2}(\mathcal{K})\right\|^{2} \leq c\left\{\left\|\kappa M\left(D_{x}, D_{y}, D_{z}, \tau\right) U ; L_{2}(\mathcal{K})\right\|^{2}+\left\|\psi U ; L_{2}(\mathcal{K})\right\|^{2}\right\} .
$$

In the role of $U$ we take the function $(x, y, z) \mapsto U^{\varepsilon}(x, y, z)=U(x / \varepsilon, y / \varepsilon, z / \varepsilon)$. Replacing $\tau$ by $\tau /(|\tau| \varepsilon)$, where $\varepsilon>0$, we rewrite the latter inequality in the form

$$
(\gamma /|\tau| \varepsilon)^{2}\left\|\kappa U^{\varepsilon} ; L_{2}(\mathcal{K})\right\|^{2} \leq c\left\{\left\|\kappa M\left(D_{x}, D_{y}, D_{z}, \tau /|\tau| \varepsilon\right) U^{\varepsilon}, L_{2}(\mathcal{K})\right\|^{2}+\left\|\psi U^{\varepsilon} ; L_{2}(\mathcal{K})\right\|^{2}\right\} .
$$

After the change of variables $(x, y, z) \mapsto \eta=(x / \varepsilon, y / \varepsilon, z / \varepsilon)$, we arrive at the estimate

$$
(\gamma /|\tau|)^{2}\left\|\kappa_{\varepsilon} U ; L_{2}(\mathcal{K})\right\|^{2} \leq c\left\{\left\|\kappa_{\varepsilon} M\left(D_{\eta}, \theta\right) U ; L_{2}(\mathcal{K})\right\|^{2}+\varepsilon^{2}\left\|\psi_{\varepsilon} U ; L_{2}(\mathcal{K})\right\|^{2}\right\}
$$

with $\kappa_{\varepsilon}(\eta)=\kappa(\varepsilon \eta)$. Multiplying this by $\varepsilon^{-2 \beta}$, putting $\varepsilon=2^{-j}, j=1,2,3, \ldots$, and adding all these inequalities, we obtain (22).

Step 3. Estimation in the intermediate zone. We add inequalities (21) and (22). Suppose $\kappa_{\infty}=1$ outside the support of $\chi$. Then, on the left-hand side, $\kappa_{\infty}$ can be dropped because

$$
(\gamma /|\tau|)\left\|\chi U ; H_{\beta}^{0}(\mathcal{K})\right\| \leq\left\|\chi U ; H_{\beta}^{0}(\mathcal{K})\right\| \leq\left\|\chi U ; H_{\beta}^{1}(\mathcal{K}, 1)\right\| .
$$


The resulting inequality takes the form

$$
\begin{aligned}
& (\gamma /|\tau|)^{2}\left\|U ; H_{\beta}^{0}(\mathcal{K})\right\|^{2}+\left\|\chi U ; H_{\beta}^{1}(\mathcal{K}, 1)\right\|^{2} \\
& \quad \leq c\left\{\left\|M\left(D_{\eta}, \theta\right) U ; H_{\beta}^{0}(\mathcal{K})\right\|^{2}+\left\|\psi_{\infty} U ; H_{\beta-1}^{0}(\mathcal{K})\right\|^{2}+\left\|\psi U ; H_{\beta}^{0}(\mathcal{K})\right\|^{2}\right\} .
\end{aligned}
$$

We estimate the last term:

$$
\left\|\psi U ; H_{\beta}^{0}(\mathcal{K})\right\|^{2} \leq \int_{|\eta|<a}|\eta|^{2 \beta}|U|^{2} d \eta=\left(\int_{0 \leq|\eta| \leq \varepsilon}+\int_{\varepsilon \leq|\eta| \leq a}\right)|\eta|^{2 \beta}|U|^{2} d \eta .
$$

The first integral does not exceed $c \varepsilon^{2}\left\|\chi U ; H_{\beta}^{1}(\mathcal{K})\right\|^{2}$. Therefore, choosing $\varepsilon$ sufficiently small, we can include this integral in the left-hand side of the inequality. The second integral is dominated by $c\left\|\psi_{\infty} U ; H_{\beta-1}^{0}(\mathcal{K})\right\|^{2}$. Now the estimate can be rewritten as

$$
\begin{aligned}
& (\gamma /|\tau|)^{2}\left\|U ; H_{\beta}^{0}(\mathcal{K})\right\|^{2}+\left\|\chi U ; H_{\beta}^{1}(\mathcal{K}, 1)\right\|^{2} \\
& \quad \leq c\left\{\left\|M\left(D_{\eta}, \theta\right) U ; H_{\beta}^{0}(\mathcal{K})\right\|^{2}+\left\|\psi_{\infty} U ; H_{\beta-1}^{0}(\mathcal{K})\right\|^{2}\right\} .
\end{aligned}
$$

After the change of variables $(x, y, z)=|\tau|^{-1} \eta$, we obtain

$$
\begin{aligned}
\gamma^{2} \| v ; & H_{\beta}^{0}(\mathcal{K})\left\|^{2}+\right\| \chi_{|\tau|} v ; H_{\beta}^{1}(\mathcal{K},|\tau|) \|^{2} \\
& \leq c\left\{\left\|M\left(D_{x}, D_{y}, D_{z}, \tau\right) v ; H_{\beta}^{0}(\mathcal{K})\right\|^{2}+\left\|\psi_{\infty,|\tau|} v ; H_{\beta-1}^{0}(\mathcal{K})\right\|^{2}\right\}
\end{aligned}
$$

where $\psi_{\infty,|\tau|}(r)=\psi_{\infty}(|\tau| r)$ and $\chi_{|\tau|}(r)=\chi(|\tau| r), v(x, y, z)=U(|\tau| x,|\tau| y,|\tau| z)$. Taking (15) and the inequality $\beta \leq 1$ into account, for the second summand we obtain

$$
\begin{aligned}
& \left\|\psi_{\infty,|\tau|} v ; H_{\beta-1}^{0}(\mathcal{K})\right\|^{2} \leq \int_{b /|\tau|<r} r^{2(\beta-1)}|v|^{2} d x d y d z \\
& \quad \leq c|\tau|^{2(1-\beta)} \int_{\mathcal{K}}|v|^{2} d x d y d z \leq c|\tau|^{2(1-\beta)} \gamma^{-2}\left\|M\left(D_{x}, D_{y}, D_{z}, \tau\right) v ; L_{2}(\mathcal{K})\right\|^{2} .
\end{aligned}
$$

This leads to (20).

We introduce the spaces $\mathcal{D} H_{\beta}(G,|\tau|)$ and $\mathcal{R} H_{\beta}(G,|\tau|)$ obtained by completion of the set $\mathcal{C}_{0}^{\infty}(\bar{G} \backslash 0)$ in the norms

$$
\begin{aligned}
\left\|v ; \mathcal{D} H_{\beta}(G,|\tau|)\right\| & =\left(\gamma^{2}\left\|v ; H_{\beta}^{0}(G)\right\|^{2}+\left\|\chi_{|\tau|} v ; H_{\beta}^{1}(G,|\tau|)\right\|^{2}\right)^{1 / 2}, \\
\left\|f ; \mathcal{R} H_{\beta}(G,|\tau|)\right\| & =\left(\left\|f ; H_{\beta}^{0}(G)\right\|^{2}+\left(|\tau|^{1-\beta} / \gamma\right)^{2}\left\|f ; L_{2}(G)\right\|^{2}\right)^{1 / 2},
\end{aligned}
$$

where $\chi_{|\tau|}(x, y, z)=\chi(|\tau| x,|\tau| y,|\tau| z)$ and $\chi \in \mathcal{C}^{\infty}(\bar{G})$ is a cut-off function equal to 1 near the conical point $O$ and vanishing outside a neighborhood in which $G$ coincides with $\mathcal{K}$. The spaces $\mathcal{D} H_{\beta}(\mathcal{K},|\tau|)$ and $\mathcal{R} H_{\beta}(\mathcal{K},|\tau|)$ are defined in a similar way. Now, (20) takes the form

$$
\left\|v ; \mathcal{D} H_{\beta}(\mathcal{K},|\tau|)\right\| \leq c\left\|M\left(D_{x}, D_{y}, D_{z}, \tau\right) v ; \mathcal{R} H_{\beta}(\mathcal{K},|\tau|)\right\| .
$$

Using (23), we prove a similar estimate in the domain $G$.

Proposition 1.4.3. Let $\beta \leq 1$. Suppose that the line $\operatorname{Im} \lambda=\beta+1 / 2$ contains no eigenvalues of the pencil $\mathfrak{A}$. If $\gamma>\gamma_{0}$ with sufficiently large $\gamma_{0}$, then for any $v \in D_{\beta}(G)$ we have the inequality

$$
\left\|v ; \mathcal{D} H_{\beta}(G,|\tau|)\right\| \leq c\left\|M\left(D_{x}, D_{y}, D_{z}, \tau\right) v ; \mathcal{R} H_{\beta}(G,|\tau|)\right\|
$$

with a constant $c$ independent of $v$ and $\tau$. 
Proof. Let $\psi \in \mathcal{C}^{\infty}(\bar{G})$ be a cut-off function equal to 1 near $O$ and vanishing outside a neighborhood in which $G$ coincides with $\mathcal{K}$. Since $v=\psi v+(1-\psi) v$, we have

$$
\left\|v ; \mathcal{D} H_{\beta}(G,|\tau|)\right\| \leq\left\|\psi v ; \mathcal{D} H_{\beta}(G,|\tau|)\right\|+\left\|(1-\psi) v ; \mathcal{D} H_{\beta}(G,|\tau|)\right\| .
$$

We estimate the first term on the right. Since $\psi v \in D_{\beta}(\mathcal{K})$, from (23) we deduce that

$$
\begin{aligned}
& \left\|\psi v ; \mathcal{D} H_{\beta}(G,|\tau|)\right\| \leq\left\|M \psi v ; \mathcal{R} H_{\beta}(\mathcal{K},|\tau|)\right\| \\
& \quad \leq c\left\{\left\|\psi M v ; \mathcal{R} H_{\beta}(\mathcal{K},|\tau|)\right\|+\left\|[M, \psi] v ; \mathcal{R} H_{\beta}(\mathcal{K},|\tau|)\right\|\right\},
\end{aligned}
$$

where $M$ stands for $M\left(D_{x}, D_{y}, D_{z}, \tau\right)$. Next, we have

$$
\begin{aligned}
& \left\|[M, \psi] v ; \mathcal{R} H_{\beta}(\mathcal{K},|\tau|)\right\| \leq\left\{\left\|[M, \psi] v ; H_{\beta}^{0}(\mathcal{K})\right\|+\left(|\tau|^{1-\beta} / \gamma\right)\left\|[M, \psi] v ; L_{2}(\mathcal{K})\right\|\right\} \\
& \quad \leq c\left\{\left\|v ; H_{\beta}^{0}(G)\right\|+\left(|\tau|^{1-\beta} / \gamma\right)\left\|v ; L_{2}(G)\right\|\right\} .
\end{aligned}
$$

Recalling (15), we conclude that

$$
\begin{gathered}
\left\|[M, \psi] v ; \mathcal{R} H_{\beta}(\mathcal{K},|\tau|)\right\| \leq c\left\{\left\|v ; H_{\beta}^{0}(G)\right\|+\left(|\tau|^{1-\beta} / \gamma\right) \cdot(1 / \gamma)\left\|M v ; L_{2}(G)\right\|\right\} \\
\leq c\left\{\left\|v ; H_{\beta}^{0}(G)\right\|+\left\|M v ; \mathcal{R} H_{\beta}(G,|\tau|)\right\|\right\}
\end{gathered}
$$

for $\gamma>1$. In its turn,

$$
\left\|\psi M v ; \mathcal{R} H_{\beta}(\mathcal{K},|\tau|)\right\| \leq c\left\|M v ; \mathcal{R} H_{\beta}(G,|\tau|)\right\| .
$$

Thus, we have estimated the first term on the right in (25):

$$
\left\|\psi v ; \mathcal{D} H_{\beta}(G,|\tau|)\right\| \leq c\left\{\left\|M v ; \mathcal{R} H_{\beta}(G,|\tau|)\right\|+\left\|v ; H_{\beta}^{0}(G)\right\|\right\}
$$

Now we pass to the second term. The definition of the norm in $\mathcal{D} H_{\beta}(G,|\tau|)$ implies that

$$
\left\|(1-\psi) v ; \mathcal{D} H_{\beta}(G,|\tau|)\right\| \leq\left\{\gamma\left\|(1-\psi) v ; H_{\beta}^{0}(G)\right\|+\left\|\chi_{|\tau|}(1-\psi) v ; H_{\beta}^{1}(G,|\tau|)\right\|\right\} .
$$

For sufficiently large $\gamma$, we have $\chi_{|\tau|}(1-\psi) \equiv 0$ because the supports of the factors do not overlap. Applying (15), we obtain

$$
\begin{aligned}
\gamma \|(1 & -\psi) v ; H_{\beta}^{0}(G)\|\leq c \gamma\|(1-\psi) v ; L_{2}(G)\|\leq c\| M(1-\psi) v ; L_{2}(G) \| \\
& \leq c\left\{\left\|(1-\psi) M v ; L_{2}(G)\right\|+\left\|[M,(1-\psi)] v ; L_{2}(G)\right\|\right\} \\
& \leq c\left\{\left\|M v ; H_{\beta}^{0}(G)\right\|+\left\|v ; H_{\beta}^{0}(G)\right\|\right\} \\
& \leq c\left\{\left\|M v ; \mathcal{R} H_{\beta}(G,|\tau|)\right\|+\left\|v ; H_{\beta}^{0}(G)\right\|\right\} .
\end{aligned}
$$

Collecting the estimates, we rewrite (25) as

$$
\left\|v ; \mathcal{D} H_{\beta}(G,|\tau|)\right\| \leq c\left\{\left\|M v ; \mathcal{R} H_{\beta}(G,|\tau|)\right\|+\left\|v ; H_{\beta}^{0}(G)\right\|\right\} .
$$

We recall the definition of the norm in $\mathcal{D} H_{\beta}(G,|\tau|)$, choose $\gamma$ sufficiently large, and include the second term in the left-hand side. As a result, we obtain (24).

1.5. The operator of the problem in a scale of weighted spaces. In the subsection, we study the operator of problem (5), (6) in some spaces related to estimates (23) and (24). We consider the case of a bounded domain $G$. The corresponding statements for the problem in $\mathcal{K}$ are formulated at the end of the subsection.

With problem (5), (6) in a bounded domain $G$ with conical point $O$, we associate the operator $v \mapsto M_{\beta}(\tau) v:=M\left(D_{x}, D_{y}, D_{z}, \tau\right) v$ with domain $D_{\beta}(G)$ and acting from $\mathcal{D} H_{\beta}(G,|\tau|)$ to $\mathcal{R} H_{\beta}(G,|\tau|)$. It is easily seen that the operator $M_{\beta}(\tau)$ admits closure (we keep the same notation for the closed operator). If the $\operatorname{line} \operatorname{Im} \lambda=\beta+1 / 2$ contains no eigenvalues of the pencil $\mathfrak{A}$ and $\beta \leq 1$, then for the functions in the domain $\mathcal{D} M_{\beta}(\tau)$ of the closed operator we have the estimate

$$
\left\|v, \mathcal{D} H_{\beta}(G,|\tau|)\right\| \leq c\left\|M_{\beta}(\tau) v, \mathcal{R} H_{\beta}(G,|\tau|)\right\| .
$$

The next proposition immediately follows from (26). 
Proposition 1.5.1. Let $\beta \leq 1$. Suppose that the line $\operatorname{Im} \lambda=\beta+1 / 2$ contains no eigenvalues of the pencil $\mathfrak{A}$. Then the kernel of the operator $\operatorname{Ker} M_{\beta}(\tau)$ is trivial, and the range $\operatorname{Im} M_{\beta}(\tau)$ is closed in $\mathcal{R} H_{\beta}(G,|\tau|)$.

Let $1 / 2>\beta_{1}>\beta_{2}>\cdots$ be all numbers in $]-\infty, 1 / 2\left[\right.$ such that the $\operatorname{line} \operatorname{Im} \lambda=\beta_{k}+1 / 2$ contains an eigenvalue of $\mathfrak{A}$. We denote by $S_{m}$ the sum of the multiplicities of all the eigenvalues of $\mathfrak{A}$ in the strip $\operatorname{Im} \lambda \in\left[\beta_{m}+1 / 2 ; \beta_{1}+1 / 2\right]$.

Definition 1.5.2. A solution of the equation $M_{\beta}(\tau) v=f$, where $f \in \mathcal{R} H_{\beta}(G,|\tau|)$, is called a strong $\beta$-solution of the problem (5), (6) with right-hand side $f$.

Theorem 1.5.3. A) Suppose $\beta \in\left[\beta_{1}, 1\right]$ and the line $\operatorname{Im} \lambda=\beta+1 / 2$ contains no eigenvalues of the pencil $\mathfrak{A}$. If $\gamma>\gamma_{0}$ with sufficiently large $\gamma_{0}$, then, for any $f \in$ $\mathcal{R H}_{\beta}(G,|\tau|)$, problem (5)), (6) with right-hand side $f$ admits a unique strong $\beta$-solution $v$, and

$$
\left\|v ; \mathcal{D} H_{\beta}(G,|\tau|)\right\| \leq c\left\|f ; \mathcal{R} H_{\beta}(G,|\tau|)\right\| .
$$

B) Assume that $\beta \in] \beta_{m+1}, \beta_{m}[$. A strong $\beta$-solution of the problem (5)), (6) with right-hand side $f \in \mathcal{R} H_{\beta}(G,|\tau|)$ exists (and is unique) if $S_{m}$ conditions $\left(f, w_{s, k}\right)_{G}=0$ are fulfilled, where $\left\{w_{s, k}\right\}_{k=-1, \ldots,-m}^{s=1, \ldots, N_{k}}$ is a basis in $\operatorname{Ker} M_{\beta}(\tau)^{*}$. Such a solution satisfies the estimate in $\mathrm{A})$.

Proof. A) Suppose that $w \in \operatorname{Ker} M_{\beta}(\tau)^{*}$, where $M_{\beta}(\tau)^{*}$ is the operator adjoint to $M_{\beta}(\tau)$ with respect to the extension of the inner product on $L_{2}(G)$. By the local properties of solutions of elliptic problems (see, e.g., [12]), $w$ belongs to $\mathcal{C}_{c}^{\infty}(\bar{G} \backslash O)$ and satisfies (17), (18). Moreover, in a neighborhood of $O$ we have an asymptotic representation of the form

$$
w \sim \chi \sum_{k} \sum_{s=1, \ldots, N_{k}} c_{s, k} V_{s, k, T}
$$

where $V_{s, k, T}$ is the sum of the first $T$ terms in the formal series (19). For $\tau$ fixed, the quantity

$$
\left(\int_{G}|v|^{2}\left(1+r^{2 \beta}\right)^{-1} d x d y d z\right)^{1 / 2}
$$

is an equivalent norm on $\mathcal{R} H_{\beta}(G,|\tau|)^{*}$. If $\beta \geq 0$, then $w \in \mathcal{R} H_{\beta}(G,|\tau|)^{*} \subset L_{2}(G)$. Since $\left(M_{\beta}(\tau) u, v\right)_{G}=\left(u, M_{\beta}(\tau)^{*} v\right)_{G}$, the asymptotics near $O$ of the functions in $\mathcal{D} M_{\beta}(\tau)^{*}$ may contain terms $V_{s, k, T}$ corresponding to the eigenvalues in the strip $\left.\operatorname{Im} \lambda \in\right] 1 / 2,3 / 2[$ only in the form of combinations $\alpha_{s, k} V_{s,-k, T}+\beta_{s, k} V_{s, k, T}$ (see the proof of Proposition 1.3.7). The coefficients $\left\{\alpha_{s, k}, \beta_{s, k}\right\}$ are the same as in the linear set $D_{\beta}(G)$ on which the operator $M_{\beta}(\tau)$ had been given initially before it was extended by closure. Then $w$ is in $L_{2}(G)$ and has an asymptotics compatible with the membership relation $w \in \mathcal{D} M(\bar{\tau})$, where the operator $M(\bar{\tau})$ is obtained by closure from the operator given initially on the lineal $D(G)$ with the same coefficients $\left\{\alpha_{s, k}, \beta_{s, k}\right\}$ as in $D_{\beta}(G)$. Using estimate (15) and Remark 1.3.3, we see that $w=0$.

Suppose $\beta \in\left[\beta_{1}, 1\right]$ and $\beta<0$. This means that the strip $\operatorname{Im} \lambda \in\left[\beta_{1}+1 / 2,3 / 2\right]$ contains no eigenvalues of the pencil $\mathfrak{A}$. The results on the asymptotics of solutions of elliptic problems (see [12]) show that for $w$ we have an asymptotic representation of the form

$$
w=\chi \sum_{s, k} c_{s, k} V_{s, k, T}+v
$$

where $v \in \mathcal{R} H_{\beta^{\prime}}(G,|\tau|)^{*}, \beta^{\prime} \in\left[0,1\left[\right.\right.$, and the sum contains the $V_{s, k, T}$ corresponding to the eigenvalues of $\mathfrak{A}$ in the strip $\operatorname{Im} \lambda \in\left[\beta+1 / 2, \beta^{\prime}+1 / 2\right]$. Since this strip contains no points of the spectrum, we have $w=v$ and $w \in \mathcal{R} H_{\beta^{\prime}}(G,|\tau|)^{*} \subset L_{2}(G)$. For $\beta^{\prime} \geq 0$, the above argument leads to $w=0$. 
B) Assume that $\beta \in] \beta_{m+1}, \beta_{m}\left[\right.$. We construct a certain collection of $S_{m}$ functions and prove that it is a basis in $\operatorname{Ker} M_{\beta}(\tau)^{*}$. This will prove the theorem, because the range of $M_{\beta}(\tau)$ is closed in $\mathcal{R} H_{\beta}(G,|\tau|)$ and the kernel is trivial. Let $M(\tau)$ and $M(\bar{\tau})$ be the closures of the differential expressions $M\left(D_{x}, D_{y}, D_{z}, \tau\right)$ and $M\left(D_{x}, D_{y}, D_{z}, \bar{\tau}\right)$, respectively, defined on the linear set $D(G)$ with $\alpha_{s, k}=0$ and $\beta_{s, k}=1$. Recall that $V_{s, k, T}$ is the sum of first $T$ terms in (19). It is easily seen that $M\left(D_{x}, D_{y}, D_{z}, \bar{\tau}\right) \chi V_{s, k, T}=$ $O\left(r^{\operatorname{Im} \lambda_{k}-2+T}\right)$ near the point $O$. We choose a sufficiently large $T$ so as to have the inclusion $M\left(D_{x}, D_{y}, D_{z}, \bar{\tau}\right) \chi V_{s, k, T}:=F_{s, k, T} \in L_{2}(G)$. For the eigenvalues of $\mathfrak{A}$ in the strip $\operatorname{Im} \lambda \in] 1 / 2,1\left[\right.$, it suffices to take the first term $V_{s, k, 1}(r, \vartheta, \varphi)=r^{i \lambda_{-k}} \Phi_{s,-k}(\vartheta, \varphi)=$ $u_{s,-k}(r, \vartheta, \varphi)$. For the eigenvalues with imaginary part at most $1 / 2$, we must take more terms. The strip $\operatorname{Im} \lambda \in\left[\beta_{m}+1 / 2, \beta_{1}+1 / 2\right]$ contains precisely $m$ eigenvalues of $\mathfrak{A}$. Let $\lambda_{k}$ be in this strip. The corresponding function $F_{s, k, T}$ is in $L_{2}(G)$. By Theorem 1.3.9, the equation $M(\bar{\tau}) w_{s, k, T}=F_{s, k, T}$ has a solution. Put $w_{s, k}:=\chi V_{s, k, T}-w_{s, k, T}$. We construct such functions for all eigenvalues of $\mathfrak{A}$ in the strip $\operatorname{Im} \lambda \in\left[\beta_{m}+1 / 2, \beta_{1}+1 / 2\right]$. For each $\lambda_{p}$ there are $N_{p}$ such functions. It is not hard to see that $w_{s, k}$ satisfies (17) and (18) and belongs to $\mathcal{R} H_{\beta}(G,|\tau|)^{*}$. We show that the $w_{s, k}$ are in the kernel of $M_{\beta}(\tau)^{*}$. Suppose $f \in \mathcal{C}^{\infty}(G) \cap \mathcal{R} H_{\beta}(G,|\tau|)$. Since $f \in \mathcal{R} H_{\beta}(G,|\tau|) \subset L_{2}(G)$, Theorem 1.3.9 implies the existence of $v \in L_{2}(G)$ such that $M(\tau) v=f$. Near the point $O$, the function $v$ admits the asymptotic representation

$$
v=\chi \sum d_{s, k} U_{s, k, T}+h
$$

where $U_{s, k, T}$ denotes the sum of the first $T$ terms of the formal series

$$
U_{s, k}(r, \vartheta, \varphi)=r^{i \lambda_{k}} \sum_{q=0}^{\infty} r^{q} \tau^{q} \Psi_{q}(\vartheta, \varphi),
$$

where $\Psi_{0}=\Phi_{s, k}$. For the linear set $D(G)$, all the coefficients $\alpha_{s, k}$ vanish; therefore, $v$ contains the terms corresponding to the eigenvalues in the $\operatorname{strip} \operatorname{Im} \lambda \in\left[\beta_{m}+1 / 2\right.$, $\beta_{1}+1 / 2\left[\right.$. The remainder $h$ is $o\left(r^{i \lambda_{m}}\right)$. The number $T$ is taken large enough that $\chi r^{i \lambda_{k}+T+1} \Psi_{T}$ decays more rapidly than $r^{i \lambda_{m}}$ as $r \rightarrow 0$. The coefficients $d_{s, k}$ are calculated by the formulas $d_{s, k}=i\left(f, w_{s, k}\right)$ (see [12] Chapters 3, 4]). The identities $\left(f, w_{s, k}\right)=0$ for all the $w_{s, k}$ that we have constructed are implied by the condition $v \in \mathcal{D} H_{\beta}(G,|\tau|)$. Thus, the $w_{s, k}$ are in the kernel of $M_{\beta}(\tau)^{*}$. We show that they form a basis in $\operatorname{Ker} M_{\beta}(\tau)^{*}$. Let $w \in \operatorname{Ker} M_{\beta}(\tau)^{*}$. Then, near the point $O$, we have

$$
w=\chi \sum c_{s, k} V_{s, k, T}+h
$$

where the sum involves the functions corresponding to the eigenvalues of $\mathfrak{A}$ in the strip $\operatorname{Im} \lambda \in\left[\beta_{m}+1 / 2, \beta_{1}+1 / 2\right]$, and the remainder $h$ is in $L_{2}(G)$. We put $z=w-\sum c_{s, k} w_{s, k}$. The function $z$ belongs to $L_{2}(G)$. The asymptotics involves the terms corresponding to the eigenvalues satisfying $\operatorname{Im} \lambda \leq 1$. Therefore, $z \in \mathcal{D} M(\bar{\tau})$. Since $M(\bar{\tau}) z=0$, we have $z=0$ and $w=\sum c_{s, k} w_{s, k}$.

Remark 1.5.4. Theorem 1.5.3 remains valid for problem (5), (6) in the cone $\mathcal{K}$.

The above proof applies almost without changes for the problem in $\mathcal{K}$. There is only one distinction. For the problem in $\mathcal{K}$, a question arises about the behavior at infinity of the functions belonging to the cokernel. Using (22), we can check that these functions decay more rapidly than any power of $r$.

1.6. The asymptotics of solutions. Suppose $f \in \mathcal{R} H_{\beta}(G,|\tau|)$ and $\left.\beta \in\right] \beta_{m+1}, \beta_{m}[$. Since $\mathcal{R} H_{\beta}(G,|\tau|) \subset L_{2}(G)$, the problem (5), (6) admits a unique strong solution $u$ (Theorem 1.3.9). By Theorem 1.5.3, this solution is in $\mathcal{D} H_{\beta}(G,|\tau|)$ provided $\left(f, w_{s, k}\right)_{G}=$ 
0 with $k=-1, \ldots,-m$ and $s=1, \ldots, N_{k}$, where $\left\{w_{s, k}\right\}_{k=-1, \ldots,-m}^{s=1, \ldots, N_{k}}$ is the basis in $\operatorname{Ker} M_{\beta}(\tau)^{*}$ constructed in the proof of Theorem 1.5.3. For any $f$, we obtain an asymptotic formula for the strong solution with remainder in $\mathcal{D} H_{\beta}(G,|\tau|)$.

Theorem 1.6.1. Assume that $\left.f \in \mathcal{R} H_{\beta}(G,|\tau|), \beta \in\right] \beta_{m+1}, \beta_{m}\left[\right.$, and $\gamma>\gamma_{0}$ with sufficiently large $\gamma_{0}$. Then the strong solution $u$ of problem (5), (6) with right-hand side $f$ admits the representation

$$
u=\chi_{|\tau|} \sum c_{s, k} U_{s, k, T}+w .
$$

Here $U_{s, k, T}$ denotes the sum of the first $T$ terms of the formal series (27), $w$ is an element of $\mathcal{D} H_{\beta}(G,|\tau|)$, and $\chi_{|\tau|}(r)=\chi(|\tau| r)$, where $\chi$ is a cut-off function equal to 1 near the point $O$ and vanishing outside a neighborhood in which the domain $G$ coincides with the cone $\mathcal{K}$. The sum consists of the terms corresponding to the eigenvalues of the pencil $\mathfrak{A}$ in the strip $\operatorname{Im} \lambda \in\left[\beta_{m}+1 / 2,1\left[\right.\right.$. The coefficients $c_{s, k}$ are given by

$$
c_{s, k}=i\left(f, w_{s, k}\right)_{G} \text {. }
$$

The estimates

$$
\begin{aligned}
\left|c_{s, k}\right| & \leq c|\tau|^{\beta+1 / 2-\operatorname{Im} \lambda_{k}}\left\|f ; \mathcal{R} H_{\beta}(G,|\tau|)\right\|, \\
\left\|w ; \mathcal{D} H_{\beta}(G,|\tau|)\right\| & \leq c(|\tau| / \gamma)\left\|f ; \mathcal{R} H_{\beta}(G,|\tau|)\right\|
\end{aligned}
$$

are valid with a constant $c$ independent of $\tau$.

Proof. As in the proof of Theorem 1.5.3, we need the operators $M(\tau, G)$ and $M(\bar{\tau}, G)$ obtained by closure of the differential expressions $M\left(D_{x}, D_{y}, D_{z}, \tau\right)$ and $M\left(D_{x}, D_{y}, D_{z}, \bar{\tau}\right)$ (respectively) on the linear set $D(G)$ with $\alpha_{s, k}=0, \beta_{s, k}=1$. Let $\left\{w_{s, k}\right\}$ denote the basis in $\operatorname{Ker} M_{\beta}(\tau, G)^{*}$ constructed in the proof of Theorem 1.5.3 with the help of the operator $M(\bar{\tau}, G)$, and let $M(\theta, \mathcal{K})$ and $M(\bar{\theta}, \mathcal{K})$ be the corresponding operators for the problem (7), (6) in the cone $\mathcal{K}$. The coefficients $\left\{\alpha_{s, k}, \beta_{s, k}\right\}$ in $D(\mathcal{K})$ are the same as in $D(G)$. We denote by $\left\{\mathcal{W}_{s, k}\right\}$ the basis in $\operatorname{Ker} M_{\beta}(\theta, \mathcal{K})^{*}$ constructed with the help of $M(\bar{\theta}, \mathcal{K})$ in the same way as the basis $\left\{w_{s, k}\right\}$ was constructed in $\operatorname{Ker} M_{\beta}(\tau, G)^{*}$ with the help of $M(\bar{\tau}, G)$. Let $\mathcal{U}_{s, k}$ stand for the formal series similar to (27) and satisfying (7), (6).

Note that the coefficients $\left\{\alpha_{s, k}, \beta_{s, k}\right\}$ indicated above were chosen for convenience only. We could take any collection obeying $\operatorname{Re} \alpha_{s, k} \overline{\beta_{s, k}}=0,\left|\alpha_{s, k}\right|+\left|\beta_{s, k}\right|>0$. This would give rise to new operators $M(\tau, G)$ and $M(\bar{\tau}, G)$ and to another basis $\left\{\widetilde{w}_{s, k}\right\}$ in $\operatorname{Ker} M_{\beta}(\tau, G)^{*}$. The new formula (28) would contain the terms $c_{s, k}\left(\alpha_{s, k} U_{s,-k, T}+\right.$ $\left.\beta_{s, k} U_{s, k, T}\right)$ corresponding to the eigenvalues of $\mathfrak{A}$ in the strip $\left.\operatorname{Im} \lambda \in\right] 1 / 2,3 / 2[$. The formulas for the coefficients $c_{s, k}$ and the estimates in the statement of the theorem do not change.

Let $u$ be a solution of the equation $M(\tau, G) u=f$, and let $h:=M(\tau, G) \chi u$. We denote by $U$ a solution of the equation $M(\theta, \mathcal{K}) U=H$ with $H(\eta)=(1 /|\tau|) h(\eta /|\tau|)$, $\eta=(|\tau| x,|\tau| y,|\tau| z)$. Since a strong solution is unique, we have $U(\eta)=\chi(\eta /|\tau|) u(\eta /|\tau|)$. The properties of solutions of elliptic problems in domains with singularities show that

$$
U(\eta)=\zeta(\eta) \sum d_{s, k} \mathcal{U}_{s, k, T}(\eta)+\mathcal{V}(\eta)
$$

in a neighborhood of $O$. This sum involves the functions $\mathcal{U}_{s, k, T}$ corresponding to the eigenvalues of $\mathfrak{A}$ in the strip $\operatorname{Im} \lambda \in\left[\beta_{m}+1 / 2,1\left[\right.\right.$, and $T$ is taken so large that $\chi r^{i \lambda_{k}+T+1} \Psi_{T+1} \in$ $H_{\beta}^{1}(\mathcal{K})$. The coefficients $d_{s, k}$ are defined by $d_{s, k}=i\left(H, \mathcal{W}_{s, k}\right)_{\mathcal{K}}$. The function $\zeta \mathcal{V}$ is in $H_{\beta}^{1}(\mathcal{K})$. We describe the properties of $\mathcal{V}$ in more detail. For this, we consider the equation $M(\theta, \mathcal{K}) \widetilde{\mathcal{V}}=\widetilde{H}$, where $\widetilde{H}=H-M\left(D_{\eta}, \theta\right)\left(\zeta \sum d_{s, k} \mathcal{U}_{s, k, T}\right)$ and the sum is the same as 
in (29). Since $\left(\widetilde{H}, \mathcal{W}_{s, k}\right)_{\mathcal{K}}=0$, Theorem 1.5.3 and Remark 1.5.4 imply $\widetilde{\mathcal{V}} \in \mathcal{D} H_{\beta}(\mathcal{K}, 1)$. But $M(\theta, \mathcal{K}) \mathcal{V}=\widetilde{H}$, and we see that $\mathcal{V}=\widetilde{\mathcal{V}}$ and $\mathcal{V} \in \mathcal{D} H_{\beta}(\mathcal{K}, 1)$

In order to estimate the coefficients $d_{s, k}$ in terms of the norm of $f$, we note that $d_{s, k}=i\left(H, \mathcal{W}_{s, k}\right)_{\mathcal{K}}$, whence

$$
\left|d_{s, k}\right| \leq c\left\|H ; \mathcal{R} H_{\beta}(\mathcal{K}, 1)\right\| \leq c|\tau|^{\beta+1 / 2}\left\|h ; \mathcal{R} H_{\beta}(\mathcal{K},|\tau|)\right\| .
$$

Since $h=\chi f+[M, \chi] u$, we obtain

$$
\left\|h ; \mathcal{R} H_{\beta}(\mathcal{K},|\tau|)\right\| \leq\left\|f ; \mathcal{R} H_{\beta}(G,|\tau|)\right\|+\left\|[M, \chi] u ; \mathcal{R} H_{\beta}(\mathcal{K},|\tau|)\right\| .
$$

If $\gamma>\gamma_{0}$ with sufficiently large $\gamma_{0}$, then

$$
\begin{aligned}
& \left\|[M, \chi] u ; \mathcal{R} H_{\beta}(\mathcal{K},|\tau|)\right\| \leq\left\|[M, \chi] u ; \mathcal{R} H_{\beta}(G,|\tau|)\right\| \\
& \quad \leq c\left\{\left\|u ; L_{2}(G)\right\|+\left(|\tau|^{1-\beta} / \gamma\right)\left\|u ; L_{2}(G)\right\|\right\} \\
& \quad \leq c\left\{(1 / \gamma)\left\|f ; L_{2}(G)\right\|+\left(|\tau|^{1-\beta} / \gamma^{2}\right)\left\|f ; L_{2}(G)\right\|\right\} \\
& \quad \leq c\left\|f ; \mathcal{R} H_{\beta}(G,|\tau|)\right\| .
\end{aligned}
$$

Thus, we have

$$
\left|d_{s, k}\right| \leq c|\tau|^{\beta+1 / 2}\left\|f ; \mathcal{R} H_{\beta}(G,|\tau|)\right\|
$$

with a constant $c$ independent of $\tau$. Since $U(\eta)=\chi(r) u(x, y, z)$, near the point $O$ we can write

$$
\chi(x, y, z) u(x, y, z)=\zeta(|\tau| r) \sum d_{s, k} \mathcal{U}_{s, k, T}(|\tau| x,|\tau| y,|\tau| z, \tau /|\tau|)+\mathcal{V}(|\tau| x,|\tau| y,|\tau| z) .
$$

Using the identity

$$
\begin{gathered}
\mathcal{U}_{s, k, T}(|\tau| x,|\tau| y,|\tau| z, \tau /|\tau|)=\sum_{q=0}^{T}(|\tau| r)^{i \lambda_{k}+q}(\tau /|\tau|)^{q} \Psi_{s}(\vartheta, \varphi) \\
=|\tau|^{i \lambda_{k}} U_{s, k, T}(r, \vartheta, \varphi, \tau),
\end{gathered}
$$

finally we obtain

$$
u(x, y, z)=\zeta(|\tau| r) \sum c_{s, k} U_{s, k, T}(x, y, z, \tau)+w(x, y, z)
$$

with $c_{s, k}=|\tau|^{i \lambda_{k}} d_{s, k}$. It is not hard to verify that $\chi w \in H_{\beta}^{1}(\mathcal{K})$ and $c_{s, k}=i\left(f, w_{s, k}\right)_{G}$. The estimate on $d_{s, k}$ obtained above leads to the inequality

$$
\left|c_{s, k}\right| \leq c|\tau|^{\beta+1 / 2-\operatorname{Im} \lambda_{k}}\left\|\mathcal{R} H_{\beta}(G,|\tau|)\right\| .
$$

Consider the remainder $w$. Since $M(\tau, G) w=\widetilde{f}$, where

$$
\widetilde{f}=f-M\left(D_{x}, D_{y}, D_{z}, \tau\right)\left(\zeta_{|\tau|} \sum c_{s, k} U_{s, k, T}\right)
$$

and $\left(\widetilde{f}, w_{s, k}\right)_{G}=0$, Theorem 1.5 .3 shows that $w \in \mathcal{D} H_{\beta}(G,|\tau|)$ and

$$
\begin{aligned}
& \left\|w ; \mathcal{D} H_{\beta}(G,|\tau|)\right\| \\
& \quad \leq c\left\{\left\|f ; \mathcal{R} H_{\beta}(G,|\tau|)\right\|+\left\|M\left(D_{x}, D_{y}, D_{z}, \tau\right)\left(\zeta_{|\tau|} \sum c_{s, k} U_{s, k, T}\right) ; \mathcal{R} H_{\beta}(G,|\tau|)\right\|\right\} .
\end{aligned}
$$

Recalling the estimate on $c_{s, k}$ and the explicit form of $M\left(D_{x}, D_{y}, D_{z}, \tau\right) U_{s, k, T}$, we majorize the last term, obtaining

$$
\left\|w ; \mathcal{D} H_{\beta}(G,|\tau|)\right\| \leq c(|\tau| / \gamma)\left\|f ; \mathcal{R} H_{\beta}(G,|\tau|)\right\| .
$$

Remark 1.6.2. Theorem 1.6.1 remains valid for problem (5), (6) in $\mathcal{K}$. 
1.7. The nonstationary problem in the cylinders $\mathcal{Q}$ and Q. Applying the inverse Fourier transform $\mathcal{F}_{\tau \rightarrow t}^{-1}$, we pass from problem (15), (6) to problem (11), (2).

Definition 1.7.1. Let $f \in V_{0}^{0}(\mathbf{Q}, \gamma)$, and let $\widehat{u}(x, y, z, \tau)$ be the strong solution of problem (5), (6) in $G$ with the right-hand side $\widehat{f}(x, y, z, \tau)=\mathcal{F}_{t \rightarrow \tau} f(x, y, z, t)$. The function $u$ defined by $u(x, y, z, t)=\mathcal{F}_{\tau \rightarrow t}^{-1} \widehat{u}(x, y, z, \tau)$ is called the strong solution of problem (11), (2) with right-hand side $f$ in $\mathbf{Q}$.

The next result follows from Theorem 1.3.9.

Theorem 1.7.2. For every $f \in V_{0}^{0}(\mathbf{Q}, \gamma)$ and every $\gamma>0$, problem (11), (2) with righthand side $f$ admits a strong solution $v$. Moreover,

$$
\gamma\left\|v ; V_{0}^{0}(\mathbf{Q}, \gamma)\right\| \leq\left\|f ; V_{0}^{0}(\mathbf{Q}, \gamma)\right\| .
$$

We fix a cut-off function $\chi \in \mathcal{C}^{\infty}(\bar{G})$ equal to 1 near $O$ and vanishing outside a neighborhood in which the domain $G$ coincides with the cone $\mathcal{K}$. We put

$$
\begin{aligned}
X u(x, y, z, t) & =\mathcal{F}_{\tau \rightarrow t}^{-1} \chi(|\tau| r) \mathcal{F}_{t^{\prime} \rightarrow \tau} u\left(x, y, z, t^{\prime}\right), \\
\Lambda^{\mu} u(x, y, z, t) & =\mathcal{F}_{\tau \rightarrow t}^{-1}|\tau|^{\mu} \mathcal{F}_{t^{\prime} \rightarrow \tau} u\left(x, y, z, t^{\prime}\right)
\end{aligned}
$$

and introduce the spaces $\mathcal{D} V_{\beta}(\mathbf{Q}, \gamma), \mathcal{R} V_{\beta}(\mathbf{Q}, \gamma)$ equipped with the norms

$$
\begin{aligned}
\left\|u ; \mathcal{D} V_{\beta}(\mathbf{Q}, \gamma)\right\| & =\left(\gamma^{2}\left\|u ; V_{\beta}^{0}(\mathbf{Q}, \gamma)\right\|^{2}+\left\|X u ; V_{\beta}^{1}(\mathbf{Q}, \gamma)\right\|^{2}\right)^{1 / 2}, \\
\left\|f ; \mathcal{R} V_{\beta}(\mathbf{Q}, \gamma)\right\| & =\left(\left\|f ; V_{\beta}^{0}(\mathbf{Q}, \gamma)\right\|^{2}+\left(1 / \gamma^{2}\right)\left\|\Lambda^{1-\beta} f ; V_{0}^{0}(\mathbf{Q}, \gamma)\right\|^{2}\right)^{1 / 2} .
\end{aligned}
$$

Definition 1.7.3. Let $f \in \mathcal{R} V_{\beta}(\mathbf{Q}, \gamma)$, and let $\widehat{u}(x, y, z, \tau)$ be the strong $\beta$-solution of the problem (5), (6) in $G$ with right-hand side $\widehat{f}(x, y, z, \tau)=\mathcal{F}_{t \rightarrow \tau} f(x, y, z, t)$. The function $u$ defined by $u(x, y, z, t)=\mathcal{F}_{\tau \rightarrow t}^{-1} \widehat{u}(x, y, z, \tau)$ is called the strong $\beta$-solution of problem (1), (2) in $\mathbf{Q}$ with right-hand side $f$.

The next result follows from Theorem 1.5 .3 .

Theorem 1.7.4. 1) For $\beta \in\left[\beta_{1}, 1\right]$, suppose that the line $\operatorname{Im} \lambda=\beta+1 / 2$ is free from the spectrum of $\mathfrak{A}$. Let $\gamma>\gamma_{0}$ with sufficiently large $\gamma_{0}$. Then problem (11), (2) with any right-hand side $f \in \mathcal{R} V_{\beta}(\mathbf{Q}, \gamma)$ admits a unique strong $\beta$-solution v. Moreover,

$$
\left\|v ; \mathcal{D} V_{\beta}(\mathbf{Q}, \gamma)\right\| \leq c\left\|f ; \mathcal{R} V_{\beta}(\mathbf{Q}, \gamma)\right\| .
$$

2) For $\beta \in] \beta_{m+1}, \beta_{m}[$, a strong $\beta$-solution of the problem (11), (2) with right-hand side $f \in \mathcal{R} V_{\beta}(\mathbf{Q}, \gamma)$ exists (and is unique) if for all $\tau=\sigma-i \gamma(\sigma \in \mathbb{R}, \gamma>0)$ the conditions $\left(\widehat{f}(\cdot, \tau), w_{s, k}(\cdot, \bar{\tau})\right)_{G}=0$ are fulfilled, where $\left\{w_{s, k}\right\}_{k=-1, \ldots,-m}^{s=1, \ldots, N_{k}}$ is a basis in $\operatorname{Ker} M_{\beta}(\tau)^{*}$. If such a solution exists, it satisfies the inequality in 1$)$.

Finally, we formulate the theorem obtained from Theorem 1.6.1 by the inverse Fourier transform. As the spatial part in (11), we take the operator $A$ that is the closure of the operator $A\left(D_{x}, D_{y}, D_{z}\right)$ on the domain $D(G)$ with $\alpha_{s, k}=0$.

Theorem 1.7.5. Suppose $f \in \mathcal{R} V_{\beta}(\mathbf{Q}, \gamma)$, where $\gamma>\gamma_{0}$ with sufficiently large $\gamma_{0}$, and $\beta \in] \beta_{m+1}, \beta_{m}[$. Then the strong solution of problem (11), (2) admits the representation

$$
u(x, y, z, t)=\sum U_{s, k, T}\left(r, \varphi, \vartheta, D_{t}\right)\left(X \check{c}_{s, k}\right)(x, y, z, t)+w(x, y, z, t),
$$

where $w \in \mathcal{D} V_{\beta}(\mathbf{Q}, \gamma)$. The sum consists of the terms corresponding to the eigenvalues of the pencil $\mathfrak{A}$ in the strip $\operatorname{Im} \lambda \in\left[\beta_{m}+1 / 2,1\left[\right.\right.$. The coefficients are defined by $\check{c}_{s, k}(t)=$ $\mathcal{F}_{\tau \rightarrow t}^{-1} c_{s, k}(\tau)$ with $c_{s, k}=i\left(\widehat{f}(\cdot, \tau), w_{s, k}(\cdot, \bar{\tau})\right)_{G}$, or, equivalently,

$$
\check{c}_{s, k}(t)=\int_{G} d x d y d z \int_{\mathbb{R}} d s\left\langle f(x, y, z, t-s), W_{s, k}(x, y, z, s)\right\rangle_{\mathbb{R}^{8}},
$$


where $W_{s, k}(x, y, z, t)=\mathcal{F}_{\tau \rightarrow t}^{-1} \overline{w_{s, k}(x, y, z, \bar{\tau})}$. Moreover,

$$
\begin{aligned}
\left\|e^{-\gamma t} \check{c}_{s, k}(\cdot) ; H^{\operatorname{Im} \lambda_{k}-\beta-1 / 2}(\mathbb{R})\right\| & \leq c\left\|f ; \mathcal{R} V_{\beta}(\mathbf{Q}, \gamma)\right\|, \\
\left\|w ; \mathcal{D} V_{\beta}(\mathbf{Q}, \gamma)\right\| & \leq(c / \gamma)\left\|\Lambda f ; \mathcal{R} V_{\beta}(\mathbf{Q}, \gamma)\right\| .
\end{aligned}
$$

The strong solutions and the strong $\beta$-solutions of problem (11), (2) in $\mathcal{Q}$ can be defined in the same way as in the cylinder $\mathbf{Q}$ (see Definitions 1.7.1 and 1.7.3).

Remark 1.7.6. All theorems in this subsection remain valid for the problem (11), (2) in $\mathcal{Q}$.

1.8. Explicit formulas for $w_{s, k}$ and $W_{s, k}$ in the case of the problem in the cone $\mathcal{K}$. By Theorem 1.6.1 and Remark 1.6.2, the strong solution $u$ of problem (5), (6) in $\mathcal{K}$ with right-hand side $f \in \mathcal{R} H_{\beta}(\mathcal{K},|\tau|)$ admits the asymptotic representation

$$
u(x, y, z)=\chi_{|\tau|}(r) \sum c_{s, k} U_{s, k, T}(x, y, z, \tau)+w(x, y, z) .
$$

The coefficients $c_{s, k}$ are defined by

$$
c_{s, k}(\tau)=\int_{\mathcal{K}} d x d y d z\left\langle f(x, y, z, \tau), w_{s, k}(x, y, z, \bar{\tau})\right\rangle_{\mathbb{C}}
$$

where $\left\{w_{s, k}\right\}_{k=-1, \ldots,-m}^{s=1, \ldots, N_{k}}$ is the same basis in $\operatorname{Ker} M_{\beta}(\tau, \mathcal{K})^{*}$ as before. We recall some properties of the functions $w_{s, k}$. They solve the homogeneous problem (5), (6) with $\bar{\tau}$ in place of $\tau$. In a neighborhood of the conical point, we have

$$
w_{s, k}=r^{i \lambda_{-k}} \Phi_{s,-k}(\vartheta, \varphi)+o\left(r^{i \lambda_{-k}}\right) .
$$

In this section, we obtain explicit formulas for the functions $w_{s, k}$ and their Fourier transforms. We denote $\overline{w_{s, k}(x, y, z, \bar{\tau})}$ by $h_{s, k}(x, y, z, \tau)$. Since $M\left(D_{x}, D_{y}, D_{z}, \bar{\tau}\right) w_{s, k}=0$, we have $M\left(D_{x}, D_{y}, D_{z},-\tau\right) h_{s, k}=0$. In the spherical coordinates $(r, \vartheta, \phi)$, the operator $A$ takes the form

$$
A(\partial)=A_{1}(\vartheta, \varphi) \partial / \partial r+\frac{1}{r} A_{2}(\vartheta, \varphi) \partial / \partial \vartheta+\frac{1}{r} A_{3}(\vartheta, \varphi) \partial / \partial \varphi .
$$

We shall seek $h_{s, k}$ in the form

$$
h_{s, k}(x, y, z, \tau)=r^{i \lambda_{-k}}\left(i \tau \xi(r) I+\xi^{\prime}(r) A_{1}(\vartheta, \varphi)\right) \Phi_{s,-k}(\vartheta, \varphi),
$$

where $\xi$ is a scalar function. Such a representation of $h_{s, k}$ is motivated by the corresponding argument for the Helmholtz equation (see [3]) and by the identity

$$
M\left(D_{x}, D_{y}, D_{z}, \tau\right) M\left(-D_{x},-D_{y},-D_{z}, \tau\right)=\Delta+\tau^{2} .
$$

We substitute the above expression for $h_{s, k}$ in the equation $(-\tau+A(D)) h_{s, k}=0$; using (10), we arrive at

$$
\xi^{\prime \prime}+\frac{2\left(i \lambda_{-k}+1\right)}{r} \xi^{\prime}+\tau^{2} \xi=0 .
$$

Choose the solution $\xi(r)=c r^{\nu} K_{\nu}(i \tau r)$ with $\nu=-\left(2 i \lambda_{k}+1\right) / 2$. The coefficient

$$
c=(i \tau)^{\nu-1} 2^{1-\nu} / \Gamma(\nu)
$$

is determined by the behavior of $h_{s, k}$ near the vertex of $\mathcal{K}$. Then

$$
h_{s, k}(x, y, z, t)=r^{i \lambda_{-k}} \frac{2^{1-\nu}}{\Gamma(\nu)}\left\{(i \tau r)^{\nu} K_{\nu}(i \tau r) I-(i \tau r)^{\nu} K_{\nu-1}(i \tau r) A_{1}(\vartheta, \varphi)\right\} \Phi_{s,-k}(\vartheta, \varphi) .
$$

We apply the inverse Fourier transform. It is known (see [14]) that

$$
2^{2 \mu} \Gamma(2 \mu+1)(p / r)^{-2 \mu} K_{2 \nu}(r p)=\int_{\mathbb{R}} \exp (-p t) P(t) d t, \quad \operatorname{Re} \mu>-1 / 4,
$$


where

$$
P(t)=\theta(t-r) \pi^{1 / 2}\left(t^{2}-r^{2}\right)^{(4 \mu-1) / 2} F\left(\mu-\nu, \mu+\nu, 2 \mu+1 / 2,1-t^{2} / r^{2}\right),
$$

and $F(a, b, c, z)$ is the hypergeometric function. Then

$$
(i \tau)^{\nu} K_{\nu}(i \tau r)=\frac{2^{-\mu}}{\Gamma(\mu+1 / 2)} r^{\nu-N} \mathcal{F}_{t \rightarrow \tau}(d / d t)^{N} \mathcal{T}_{N}(r, t, \mu, \nu)
$$

where

$$
\begin{array}{r}
\mathcal{T}_{N}(r, t, \mu, \nu)=\theta(t-r) \pi^{1 / 2}\left(t^{2}-r^{2}\right)^{\mu-1 / 2} F\left((\mu-\nu) / 2,(\mu+\nu) / 2, \mu+1 / 2,1-t^{2} / r^{2}\right), \\
\mu=[\nu]-\nu+m, \quad N=[\nu]+m,
\end{array}
$$

and $m$ is an arbitrary positive integer. The Fourier transform and differentiation are understood in the sense of distributions.

Thus, in accordance with Theorem 1.7 .5 and Remark 1.7.6, the strong solution $\mathcal{U}$ of problem (1), (2) in $\mathcal{Q}$ with right-hand side $\mathcal{F} \in \mathcal{R} V_{\beta}(\mathcal{Q}, \gamma)$ admits the asymptotic representation

$$
\mathcal{U}(x, y, z, t)=\sum U_{s, k, T}\left(r, \varphi, \vartheta, D_{t}\right)\left(X \check{c}_{s, k}\right)(x, y, z, t)+w(x, y, z, t),
$$

where

Moreover,

$$
\check{c}_{s, k}(t)=\int_{\mathcal{K}} d x d y d z \int_{\mathbb{R}} d s\left\langle\mathcal{F}(x, y, z, t-s), W_{s, k}(x, y, z, s)\right\rangle_{\mathbb{R}^{8}}
$$

$$
\begin{aligned}
& W_{s, k}(x, y, z, t)=\mathcal{F}_{\tau \rightarrow t}^{-1} h_{s, k}(x, y, z, \tau) \\
& =r^{i \lambda_{-k}} \frac{2^{1-\nu-\mu}}{\Gamma(\nu) \Gamma(\mu+1 / 2)} r^{\nu-\mu} \\
& \quad \times\left\{(d / d t)^{N} \mathcal{T}_{N}(r, t, \mu, \nu)-(d / d t)^{N} \mathcal{T}_{N-1}(r, t, \mu, \nu-1) A_{1}(\vartheta, \varphi)\right\} \Phi_{s,-k}(\vartheta, \varphi) .
\end{aligned}
$$

We discuss some properties of $\check{c}_{s, k}$ that follow from those of $W_{s, k}$ and from the formula for $\check{c}_{s, k}$. Observe that $\operatorname{supp} W_{s, k}=\{(x, y, z, t) \in \mathcal{K} \times \mathbb{R}: r<t\}$, and $\operatorname{sing} \operatorname{supp} W_{s, k}=$ $\{(x, y, z, t) \in \mathcal{K} \times \mathbb{R}: r=t\}$. Suppose the right-hand side $\mathcal{F}$ is a smooth function with $\operatorname{supp} \mathcal{F} \subset\left\{(x, y, z, t) \in \mathcal{K} \times \mathbb{R}: R_{1}<r<R_{2}, t>0\right\}$. Then the coefficients $\check{c}_{s, k}$ are smooth, and $\check{c}_{s, k}(t)=0$ for $t<R_{1}$. Thus, we have the "forward edge" phenomenon at the level of the coefficients. Now, suppose that the singular support sing supp $\mathcal{F}$ of the right-hand side $\mathcal{F}$ is located in the set $\left\{(x, y, z, t): R_{1}<r<R_{2}, 0<t<t_{0}\right\}$. Then the $\check{c}_{s, k}(t)$ vanish for $t<R_{1}$ and are smooth for $t>t_{0}+R_{2}$. In other words, the "back edge" phenomenon occurs: the coefficients become smooth after the vertex of $\mathcal{K}$ is abandoned by the perturbation coming from the singular support of the right-hand side.

\section{§2. The PROBLEM IN A WEDGE AND IN A WAVEGUIDE WITH EDGE}

2.1. Preliminaries. Statement of the problem. Let $\mathbb{K}=\{(r, \varphi): r>0,|\varphi|<\alpha\}$ be an angle of opening $2 \alpha$ on the plane $\mathbb{R}_{x, y}^{2}$, where $(r, \varphi)$ are the polar coordinates centered at $O$. We denote by $\mathbb{D}=\mathbb{K} \times \mathbb{R}$ the wedge with the edge $M=O \times \mathbb{R}$. We introduce some function spaces. For $s \in \mathbb{N}, \beta \in \mathbb{R}$, let $H_{\beta}^{s}(\mathbb{K})$ and $H_{\beta}^{s}(\mathbb{D})$ denote the spaces obtained from $C_{c}^{\infty}(\overline{\mathbb{K}} \backslash O)$ and $C_{c}^{\infty}(\overline{\mathbb{D}} \backslash M)$ by completion with respect to the norms

$$
\begin{aligned}
\left\|u ; H_{\beta}^{s}(\mathbb{K})\right\| & =\left(\sum_{|\alpha| \leq s} \int_{\mathbb{K}} r^{2(\beta+|\alpha|-s)}\left|D_{x, y}^{\alpha} u(x, y)\right|^{2} d x d y\right)^{1 / 2}, \\
\left\|v ; H_{\beta}^{s}(\mathbb{D})\right\| & =\left(\sum_{|\alpha| \leq s} \int_{\mathbb{D}} r^{2(\beta+|\alpha|-s)}\left|D_{x, y, z}^{\alpha} v(x, y, z)\right|^{2} d x d y d z\right)^{1 / 2} .
\end{aligned}
$$


(Here and in the sequel, $r=\left(x^{2}+y^{2}\right)^{1 / 2}$.) For $q>0$, the spaces $H_{\beta}^{s}(\mathbb{K}, q), H_{\beta}^{s}(\mathbb{D}, q)$ are equipped with the norms

$$
\begin{aligned}
\left\|u ; H_{\beta}^{s}(\mathbb{K}, q)\right\| & =\left(\sum_{k=0}^{s} q^{2 k}\left\|u ; H_{\beta}^{s-k}(\mathbb{K})\right\|^{2}\right)^{1 / 2}, \\
\left\|u ; H_{\beta}^{s}(\mathbb{D}, q)\right\| & =\left(\sum_{k=0}^{s} q^{2 k}\left\|u ; H_{\beta}^{s-k}(\mathbb{D})\right\|^{2}\right)^{1 / 2} .
\end{aligned}
$$

Now, we define function spaces in the cylinder $\mathbf{T}=\mathbb{D} \times \mathbb{R}$. Let $H_{\beta}^{s}(\mathbf{T})$ stand for the completion of $C_{c}^{\infty}((\overline{\mathbb{D}} \backslash M) \times \mathbb{R})$ in the norm

$$
\left\|w ; H_{\beta}^{s}(\mathbf{T})\right\|=\left(\sum_{|\alpha| \leq s} \int_{\mathbb{D}} \int_{\mathbb{R}} r^{2(\beta+|\alpha|-s)}\left|D_{x, y, z, t}^{\alpha} w(x, y, z, t)\right|^{2} d x d y d z d t\right)^{1 / 2} .
$$

For $q>0$, the space $H_{\beta}^{s}(\mathbf{T}, q)$ is equipped with the norm

$$
\left\|u ; H_{\beta}^{s}(\mathbf{T}, q)\right\|=\left(\sum_{k=0}^{s} q^{2 k}\left\|u ; H_{\beta}^{s-k}(\mathbf{T})\right\|^{2}\right)^{1 / 2} .
$$

Finally, for $\gamma>0, V_{\beta}^{s}(\mathbf{T}, \gamma)$ denotes the space with the norm

$$
\left\|w ; V_{\beta}^{s}(\mathbf{T}, \gamma)\right\|=\left\|w^{\gamma} ; H_{\beta}^{s}(\mathbf{T}, \gamma)\right\|,
$$

where $w^{\gamma}=e^{-\gamma t} w$.

Let $\Omega \subset \mathbb{R}^{2}$ be a bounded domain with a corner point $O$. We assume that the domain $\Omega$ coincides with $\mathbb{K}$ in a neighborhood of $O$. Off the point $O$, the boundary of $\Omega$ is smooth. We denote by $\Sigma$ the waveguide $\Omega \times \mathbb{R}$ with edge $O \times \mathbb{R}$, and by $\mathcal{T}$ the cylinder $\Sigma \times \mathbb{R}$. In the domains $\Omega, \Sigma$, and $\mathcal{T}$, we shall consider function spaces similar to those defined in $\mathbb{K}, \mathbb{D}$, and $\mathbf{T}$.

We turn to problem (11), (2) in $\mathbf{T}$ and $\mathcal{T}$. Assume that $\tau=\sigma-i \gamma, \sigma \in \mathbb{R}, \gamma>0$, and $\xi \in \mathbb{R}$. Applying the Fourier transform $\mathcal{F}_{(z, t) \rightarrow(\xi, \tau)}$ to (11), (2), we obtain a problem in $\mathbb{K}$ or in $\Omega$

$$
\tau \widehat{\mathcal{U}}+A\left(D_{x}, D_{y}, \xi\right) \widehat{\mathcal{U}}=\widehat{\mathcal{F}}
$$

with parameter $(\xi, \tau)$ and with the boundary conditions

$$
\vec{n} \times \widehat{\vec{u}}=0, \quad(\vec{n} \cdot \widehat{\vec{v}})=0, \quad \widehat{h}=0 .
$$

When dealing with the problem in $\mathbb{K}$, we put $\eta=(p x, p y)$, where $p=\left(|\tau|^{2}+|\xi|^{2}\right)^{1 / 2}$. Denoting $(\xi / p, \tau / p)$ by $\theta$, we introduce the notation $M\left(D_{\eta}, \theta\right)=\tau / p+A\left(D_{\eta}, \xi / p\right)$, $U(\eta, \xi, \tau)=\widehat{\mathcal{U}}\left(p^{-1} \eta, \xi, \tau\right)$, and $F(\eta,, \xi, \tau)=p^{-1} \widehat{\mathcal{F}}\left(p^{-1} \eta, \xi, \tau\right)$, and rewrite (30) in the form

$$
M\left(D_{\eta}, \theta\right) U=F .
$$

We also need the function space $E_{\beta}^{l}(\mathbb{K})$ obtained from $C_{c}^{\infty}(\overline{\mathbb{K}} \backslash O)$ by completion in the norm

$$
\left\|u ; E_{\beta}^{l}(\mathbb{K})\right\|=\left(\sum_{|\alpha|<l}\left\|r^{\beta}\left(1+r^{|\alpha|-l}\right) D_{x, y}^{\alpha} U ; L_{2}(\mathbb{K})\right\|^{2}\right)^{1 / 2} .
$$

Observe that the norms $\left\|\cdot ; E_{\beta}^{l}(\mathbb{K})\right\|$ and $\left\|\cdot ; H_{\beta}^{l}(\mathbb{K}, 1)\right\|$ are equivalent. The notation $E_{\beta}^{l}(\mathbb{K})$ was used in [12]; we introduced the spaces $E_{\beta}^{l}(\mathbb{K})$ for convenience of references. The next assertion was proved in [12, Lemma 6.1.2]. 
Proposition 2.1.1. The norms $\left\|w ; H_{\beta}^{l}(\mathbb{D})\right\|$ and

$$
\left(\int_{\mathbb{R}}|\xi|^{2(l-\beta)-2}\left\|W(\cdot, \xi) ; E_{\beta}^{l}(\mathbb{K})\right\|^{2} d \xi\right)^{1 / 2},
$$

where $W(\zeta, \xi)=\left(\mathcal{F}_{z \rightarrow \xi} w\right)\left(|\xi|^{-1} \zeta, \xi\right)$ and $\zeta=(|\xi| x,|\xi| y)$, are equivalent.

2.2. Operator pencil. We define the operator pencil

$$
\mathfrak{B}(\lambda) \Phi(\varphi)=r^{1-i \lambda} A\left(D_{x}, D_{y}, 0\right) r^{i \lambda} \Phi(\varphi)
$$

for the functions $\Phi \in H^{1}[-\alpha, \alpha]$ such that $r^{i \lambda} \Phi(\varphi)$ satisfy (31) on $\partial \mathbb{K}$. If $\Phi(\varphi)=$ $(\vec{u}(\varphi), \vec{v}(\varphi), h(\varphi), q(\varphi))$, then the boundary conditions can be written as

$$
\left\{\begin{array}{c}
h(\alpha)=h(-\alpha)=u_{3}(\alpha)=u_{3}(-\alpha)=0, \\
u_{1}(\alpha) \cos \alpha+u_{2}(\alpha) \sin \alpha=0, \\
u_{1}(-\alpha) \cos \alpha-u_{2}(-\alpha) \sin \alpha=0, \\
v_{1}(\alpha) \sin \alpha-v_{2}(\alpha) \cos \alpha=0, \\
v_{1}(-\alpha) \sin \alpha+v_{2}(-\alpha) \cos \alpha=0 .
\end{array}\right.
$$

In the polar coordinates, the operator $A\left(D_{x}, D_{y}, 0\right)$ takes the form

$$
A\left(D_{x}, D_{y}, 0\right)=A_{1}(\varphi) D_{r}+r^{-1} A_{2}(\varphi) D_{\varphi},
$$

where $A_{1}$ and $A_{2}$ are $(8 \times 8)$-matrices. Let $G$ denote the matrix $A(0,0,1)$. We have

$$
\begin{gathered}
A_{1} \cdot A_{1}=I, \quad A_{2} \cdot A_{2}=I, \quad A_{1} \cdot A_{2}+A_{2} \cdot A_{1}=0, \\
\frac{d A_{1}}{d \varphi}=A_{2}, \quad \frac{d A_{2}}{d \varphi}=-A_{1}, \\
G \cdot G=I, \quad A_{1} \cdot G+G \cdot A_{1}=0, \quad G \cdot A_{2}+A_{2} \cdot G=0 .
\end{gathered}
$$

The eigenfunctions and eigenvalues of the pencil $\mathfrak{B}$ are determined by the equation $\mathfrak{B}(\lambda) \Phi=0$. Rewriting this explicitly, and taking (33) into account, we obtain

$$
\lambda A_{1}(\varphi) \Phi(\varphi)+A_{2}(\varphi) D_{\varphi} \Phi(\varphi)=0 .
$$

The formulas for $A_{1}$ and $A_{2}$ and the boundary conditions for $\Phi$ show that the eigenfunctions and eigenvalues of $\mathfrak{B}$ can be found by solving two Sturm-Liouville problems for the system

$$
\left\{\begin{array}{l}
d a / d \varphi+i \lambda b=0 \\
d b / d \varphi-i \lambda a=0
\end{array}\right.
$$

The boundary conditions for these problems are as follows:

$$
\begin{aligned}
& \text { 1) } a(\alpha)=a(-\alpha)=0, \\
& \text { 2) } a(\alpha) \sin \alpha-b(\alpha) \cos \alpha=0, a(-\alpha) \sin \alpha+b(-\alpha) \cos \alpha=0 .
\end{aligned}
$$

Thus, the spectrum of $\mathfrak{B}$ consists of two sequences of eigenvalues:

$$
\lambda_{k, 1}=i \frac{\pi k}{2 \alpha}, \quad \lambda_{k, 2}=i\left(\frac{\pi k}{2 \alpha}+1\right), \quad k \in \mathbb{Z} .
$$

By using the relationship between the above Sturm-Liouville problems and equation (35), it can be shown that two linearly independent eigenfunctions correspond to every eigenvalue, and that there are no root functions. Moreover, the eigenvalues $\lambda_{k, 1}$ correspond to the components $h, q, u_{3}$, and $v_{3}$ while the $\lambda_{k, 2}$ correspond to $u_{1}, u_{2}, v_{1}$, and $v_{2}$. The eigenvalues of $\mathfrak{B}$ are located symmetrically with respect to the point $i / 2$. If $i / 2$ is an eigenvalue, then we denote it by $\lambda_{0}$. We assume that the $\lambda_{k}$ with $k>0$ denote the eigenvalues of $\mathfrak{B}$ with $\operatorname{Im} \lambda_{k}>1 / 2$ and enumerate them in such a way that the imaginary parts increase with $k$. By $\lambda_{-k}$ with $k>0$ we denote the eigenvalue symmetric to $\lambda_{k}$ 
with respect to the point $i / 2$. The same argument as in Subsection 1.2 shows that the eigenfunctions $\left\{\Phi_{s, \mp k}\right\}_{s=1,2}$ corresponding to $\lambda_{\mp k}$ can be chosen so that they satisfy the orthogonality and normalization conditions

$$
\int_{-\alpha}^{\alpha}\left\langle\partial_{\lambda} \mathfrak{B}\left(\lambda_{k}\right) \Phi_{s, k}, \Phi_{p,-k}\right\rangle d \varphi=\delta_{s, p}
$$

If $\Phi$ is an eigenfunction of $\mathfrak{B}$ corresponding to $\lambda_{k}$, then $G \Phi$ is also an eigenfunction corresponding to the same eigenvalue $\lambda_{k}$.

The properties of the pencil $\mathfrak{B}$ discussed in this subsection are summarized in the proposition below.

Proposition 2.2.1. 1) Two linearly independent eigenfunctions $\left\{\Phi_{s, k}\right\}_{s=1,2}$ satisfying $G \Phi_{1, k}=\Phi_{2, k}$ correspond to every eigenvalue $\lambda_{k}$ of $\mathfrak{B}$ with $k \neq 0$. There are no root functions. The eigenfunctions can be chosen so that

$$
\int_{-\alpha}^{\alpha}\left\langle A_{1}(\varphi) \Phi_{s, k}(\varphi), \Phi_{p, m}(\varphi)\right\rangle d \varphi=\delta_{s, p} \cdot \delta_{k,-m} .
$$

2) If $\alpha=\pi$, then $\lambda_{0}=i / 2$ is an eigenvalue of the pencil $\mathfrak{B}$. There are four linearly independent eigenfunctions $\left\{\Phi_{s, \mp 0}\right\}_{s=1,2}$ corresponding to this eigenvalue. These functions can be chosen so that they satisfy the orthogonality and normalization conditions

$$
\begin{gathered}
\int_{-\alpha}^{\alpha}\left\langle A_{1}(\varphi) \Phi_{s, \mp 0}(\varphi), \Phi_{p, \pm 0}(\varphi)\right\rangle d \varphi=\delta_{s, p}, \\
\int_{-\alpha}^{\alpha}\left\langle A_{1}(\varphi) \Phi_{s, \pm 0}(\varphi), \Phi_{p, \pm 0}(\varphi)\right\rangle d \varphi=0 \\
\int_{-\alpha}^{\alpha}\left\langle A_{1}(\varphi) \Phi_{s, k}(\varphi), \Phi_{p, \pm 0}(\varphi)\right\rangle d \varphi=0
\end{gathered}
$$

for $k \neq 0$.

We put

$$
v_{s, k}=r^{i \lambda_{k}} \Phi_{s, k}, \quad v_{s, \mp 0}=r^{i \lambda_{0}} \Phi_{s, \mp 0} .
$$

The functions $v_{s, k}$ solve the boundary value problem $A\left(D_{x}, D_{y}, 0\right) v_{s, k}=0, \Gamma v_{s, k}=0$ in $\mathbb{K}$.

2.3. On the properties of the operator $A(D)$. Consider the following elliptic problem in the wedge $\mathbb{D}$ :

$$
\left\{\begin{array}{rlrl}
A\left(D_{x}, D_{y}, D_{z}\right) \mathcal{U}(x, y, z) & =\mathcal{F}(x, y, z), & & (x, y, z) \in \mathbb{D}, \\
\Gamma \mathcal{U}(x, y, z)=\mathcal{H}(x, y, z), & & (x, y, z) \in \partial \mathbb{D} \backslash M .
\end{array}\right.
$$

In this section, we study the operator $\mathcal{A}_{\beta}$ for problem 38 ,

$$
\mathcal{A}_{\beta}=\{A(D), \Gamma\}: H_{\beta}^{1}(\mathbb{D}) \rightarrow H_{\beta}^{0}(\mathbb{D}) \times H_{\beta}^{1 / 2}(\partial \mathbb{D}),
$$

and find the numbers $\beta$ for which this operator is an isomorphism. Applying the Fourier transform $\mathcal{F}_{z \rightarrow \xi}$ to (38), we obtain a family

$$
\left\{\begin{array}{rlrl}
A\left(D_{x}, D_{y}, \xi\right) \widehat{\mathcal{U}}(x, y, \xi) & =\widehat{\mathcal{F}}(x, y, \xi), & & (x, y) \in \mathbb{K}, \\
\Gamma \widehat{\mathcal{U}}(x, y, \xi)=\widehat{\mathcal{H}}(x, y, \xi), & (x, y) \in \partial \mathbb{K} \backslash O,
\end{array}\right.
$$

of problems in $\mathbb{K}$. The new variables

$$
\begin{gathered}
\eta=|\xi|(x, y), \quad \omega=\xi /|\xi|, \\
U(\eta, \xi)=\widehat{\mathcal{U}}(\eta /|\xi|, \xi), \quad F(\eta, \xi)=|\xi|^{-1} \widehat{\mathcal{F}}(\eta /|\xi|, \xi), \quad H(\eta, \xi)=\widehat{\mathcal{H}}(\eta /|\xi|, \xi)
\end{gathered}
$$


allow us to rewrite (39) in the form

$$
\left\{\begin{aligned}
A\left(D_{\eta}, \omega\right) U(\eta, \xi) & =F(\eta, \xi), & & \eta \in \mathbb{K}, \\
\Gamma U(\eta, \xi) & =H(\eta, \xi), & & \eta \in \partial \mathbb{K} \backslash O .
\end{aligned}\right.
$$

Consider the operator

$$
\mathcal{A}_{\beta}(\omega)=\left\{A\left(D_{\eta}, \omega\right), \Gamma\right\}: E_{\beta}^{1}(\mathbb{K}) \rightarrow E_{\beta}^{0}(\mathbb{K}) \times E_{\beta}^{1 / 2}(\partial \mathbb{K}) .
$$

Theorem 2.3.1. If $\alpha<\pi$, then the operator $\mathcal{A}_{\beta}$ is an isomorphism for $\left.\beta \in\right] \max \{0,1-$ $\pi / 2 \alpha\}, \min \{1, \pi / 2 \alpha\}[$.

Proof. The operator $\mathcal{A}_{\beta}$ is an isomorphism if and only if $\mathcal{A}_{\beta}(\omega)$ is an isomorphism for $\omega=\mp 1$ (see [12 Theorem 6.2.1]). The operator $\mathcal{A}_{\beta}(\omega)$ is Fredholm if and only if the line $\operatorname{Im} \lambda=\beta$ is free from the spectrum of the pencil $\mathfrak{B}$ (see [12] Theorem 6.2.3]). We show that the kernel and the cokernel of $\mathcal{A}_{\beta}(\omega)$ are trivial if $\beta$ lies in some interval $\operatorname{Im} \lambda_{k}<\beta<\operatorname{Im} \lambda_{k+1}$. It follows that $\mathcal{A}_{\beta}(\omega)$ is an isomorphism for such $\beta$. Outside the above interval, the operator $\mathcal{A}_{\beta}(\omega)$ is not an isomorphism (see [12] Subsection 6.3]).

Let $U$ be a solution in $E_{\beta}^{1}(\mathbb{K})$ of the homogeneous problem (40). By [12, Proposition 6.2.6], we have $\chi U \in E_{\gamma}^{l}(\mathbb{K})$, where $\chi$ is an arbitrary $C^{\infty}(\overline{\mathbb{K}})$-function vanishing near the vertex, and the numbers $l \geq 1$ and $\gamma \in \mathbb{R}$ are also arbitrary. Applying $A\left(-D_{\eta}, \omega\right)$ to the relation $A\left(D_{\eta}, \omega\right) U=0$, we obtain $\left(-\Delta_{\eta}+1\right) U=0$. Let $(\vec{u}, \vec{v}, h, q)$ denote the components of $U$. We want to find conditions on $\beta$ ensuring that $h=0$. For the function $h$ we have

$$
\left\{\begin{aligned}
\left(-\Delta_{\eta}+1\right) h & =0, & & \eta \in \mathbb{K}, \\
h & =0, & & \eta \in \partial \mathbb{K} \backslash O .
\end{aligned}\right.
$$

We multiply the equation $\left(-\Delta_{\eta}+1\right) h=0$ by $h$ and integrate over $\mathbb{K}_{\varepsilon}=\{\eta \in \mathbb{K}:|\eta|>\varepsilon\}$. Integrating by parts and recalling the boundary condition, we arrive at the identity

$$
\int_{\mathbb{K}_{\varepsilon}}\left(|h|^{2}+|\nabla h|^{2}\right) d \eta_{1} d \eta_{2}=\varepsilon \int_{-\alpha}^{\alpha} h \cdot \frac{\partial h}{\partial \rho} d \phi,
$$

where $(\rho, \phi)$ are the polar coordinates on the plane $\mathbb{R}_{\eta}^{2}$. Let $\chi$ be a cut-off function equal to 1 near the vertex. We have $\chi h \in H_{\beta}^{1}(\mathbb{K})$. By [12, Theorem 4.2.1], the asymptotic representation

$$
h=\sum_{k} c_{s, k} \rho^{i \lambda_{k}} H_{s, k}+R,
$$

is valid in a neighborhood of the vertex, where $\chi R \in H_{\gamma}^{1}(\mathbb{K}), 1>\beta-\gamma>0$, and $\lambda_{k}$ is an eigenvalue of $\mathfrak{B}$ among the eigenvalues $\{\pi m / 2 \alpha\}_{m \in \mathbf{Z} \backslash 0}$. The sum in (42) consists of the terms corresponding to such eigenvalues in the strip $\operatorname{Im} \lambda \in] \gamma, \beta\left[\right.$, and the $H_{s, k}$ are the components of the eigenfunctions $\Phi_{s, k}$ of $\mathfrak{B}$ corresponding to $h$. In order that the right-hand side of (41) tend to 0 as $\varepsilon \rightarrow 0$, we must require that $\operatorname{Im} \lambda_{k}<0$ for all terms in (42). Since $h \in E_{\beta}^{1}(\mathbb{K})$, we have $\operatorname{Im} \lambda_{k}<\beta$. Assume that $\beta<\pi / 2 \alpha$. Then $\operatorname{Im} \lambda_{k} \leq-\pi / 2 \alpha$ for all terms in (42). Therefore, $h=0$ in $\mathbb{K}$. Similarly, $u_{3}$ vanishes in $\mathbb{K}$ for the same $\beta$. Now the relation $A\left(D_{\eta}, \omega\right) U=0$ implies that

$$
\begin{array}{ll}
D_{\eta_{1}} u_{1}+D_{\eta_{2}} u_{2}=-\omega u_{3}=0, & \eta \in \mathbb{K}, \\
D_{\eta_{2}} v_{1}-D_{\eta_{1}} v_{2}=-\omega h=0, & \eta \in \mathbb{K} .
\end{array}
$$

We consider $u_{1}, u_{2}$ and put $\vec{w}=\left(u_{1}, u_{2}, 0\right)$. The equation $\left(-\Delta_{\eta}+1\right) \vec{w}=0$ can be rewritten as

$$
\operatorname{curl} \operatorname{curl} \vec{w}-\nabla \operatorname{div} \vec{w}+\vec{w}=0 \text {. }
$$


We calculate the inner product of this expression and $\vec{w}$ and integrate it over $\mathbb{K}_{\varepsilon} \times I_{\eta_{3}}$, where $I=[0,1]$. Using the boundary conditions $\vec{u} \times \vec{n}=0$ and $D_{\eta_{1}} u_{1}+D_{\eta_{2}} u_{2}=0$ on $\partial \mathbb{D}$, and applying the Stokes formula, we obtain

$$
\int_{\mathbb{K}_{\varepsilon}}\left(|\operatorname{curl} \vec{w}|^{2}+|\operatorname{div} \vec{w}|^{2}+|\vec{w}|^{2}\right) d \eta_{1} d \eta_{2}=\varepsilon \int_{-\alpha}^{\alpha}((\operatorname{curl} \vec{w} \cdot[\vec{n} \times \vec{w}])+\operatorname{div} \vec{w} \cdot(\vec{n} \cdot \vec{w})) d \phi,
$$

where $\vec{n}=(\cos \phi, \sin \phi, 0)$. Let $\lambda_{k_{0}}$ be the only eigenvalue of $\mathfrak{B}$ that belongs to the sequence $\{i(\pi m / 2 \alpha+1)\}_{m \in \mathbb{Z}}$ and is such that the strip $\left.\operatorname{Im} \lambda \in\right] \operatorname{Im} \lambda_{k_{0}}, \beta[$ contains no other elements of that sequence. Near the vertex, we have the asymptotic representation

$$
\vec{w}=\sum_{s=1}^{N_{k_{0}}} c_{s, k_{0}}\left(r^{i \lambda_{k_{0}}} \vec{W}_{s, k_{0}}+r^{i \lambda_{k_{0}}+1} \tau \vec{\Psi}_{s}\right)+\sum_{k} c_{s, k} r^{i \lambda_{k}} \vec{W}_{s, k}+R .
$$

The third component of every term in the first sum vanishes, and the first two components are equal to those of the two-term partial sum $U_{s, k_{0}, 2}$ of the formal series (27). The first two components of $\vec{W}_{s, k}$ are equal to those of $\Phi_{s, k}$, and the third component vanishes. The terms of the second sum correspond to the eigenvalues $\lambda_{k}$ of $\mathfrak{B}$ that belong to the sequence $\{i(\text { pim } / 2 \alpha+1)\}_{m \in \mathbb{Z}}$ and are located in the strip $\left.\operatorname{Im} \lambda \in\right] \operatorname{Im} \lambda_{k_{0}}-1, \operatorname{Im} \lambda_{k_{0}}[$. The function $R$ is a remainder. Using the definition of $\mathfrak{B}$, it is not hard to check that $\operatorname{curl} r^{i \lambda_{k}} \vec{W}_{s, k}=0$ and $\operatorname{div} r^{i \lambda_{k}} \vec{W}_{s, k}=0$. Therefore, the right-hand side in (43) behaves as $\varepsilon^{1-2 \operatorname{Im} \lambda_{k}}$, i.e., it tends to zero as $\varepsilon \rightarrow 0$ under the condition $\operatorname{Im} \lambda_{k_{0}}<1 / 2$. This condition is fulfilled if $\beta<1$. Consequently, for $\beta<1$ we have $u_{1}=u_{2}=0$ in $\mathbb{K}$. The same argument shows that if $\beta<1$, then $v_{1}=v_{2}=0$. The relation $A\left(D_{\eta}, \omega\right) U=0$ implies that

$$
\begin{aligned}
-D_{\eta_{2}} u_{1}+D_{\eta_{1}} u_{2} & =-\omega q=0, \\
D_{\eta_{1}} v_{1}+D_{\eta_{2}} v_{2} & =-\omega v_{3}=0 .
\end{aligned}
$$

Thus, if $\beta<\min \{1, \pi / 2 \alpha\}$, then the kernel of $\mathcal{A}_{\beta}(\omega)$ is trivial. Consider the adjoint operator $\mathcal{A}_{\beta}(\omega)^{*}$. Let $V \in \operatorname{Ker} \mathcal{A}_{\beta}(\omega)^{*}$. By [12] Theorem 6.3.3], we have $V \in \operatorname{Ker} \mathcal{A}_{1-\beta}(\omega)$. Hence, $V \equiv 0$ if $1-\beta<\min \{1, \pi / 2 \alpha\}$. Thus, if

$$
\max \{0,1-\pi / 2 \alpha\}<\beta<\min \{1, \pi / 2 \alpha\},
$$

then the operator $\mathcal{A}_{\beta}(\omega)$ is an isomorphism. The operator $\mathcal{A}_{\beta}$ turns out to be an isomorphism under the same condition.

We note that if $2 \alpha<\pi$, then $\mathcal{A}_{\beta}$ is an isomorphism for $\left.\beta \in\right] 0,1$. If $\pi \leq 2 \alpha<2 \pi$, then $\mathcal{A}_{\beta}$ is an isomorphism for $\left.\beta \in\right] 1-\pi / 2 \alpha, \pi / 2 \alpha[$. If $2 \alpha=2 \pi$, then there are no $\beta$ such that $\mathcal{A}_{\beta}$ is an isomorphism.

2.4. Estimation of solutions of problems in a wedge and in an angle. In this section, we prove a global energy estimate and a weighted combined estimate for the solutions of (30), (31) in $\mathbb{K}$ and in $\Omega$. We drop some proofs similar to those for the problems in $\mathcal{K}$ and in $G$.

Proposition 2.4.1. Let $v$ be $a C_{c}^{\infty}(\overline{\mathbb{K}} \backslash O)$-function satisfying the boundary conditions (31). Then

$$
\gamma\left\|v ; L_{2}(\mathbb{K})\right\| \leq\left\|M\left(D_{x}, D_{y}, \xi, \tau\right) v ; L_{2}(\mathbb{K})\right\|,
$$

where $M\left(D_{x}, D_{y}, \xi, \tau\right)=\tau+A\left(D_{x}, D_{y}, \xi\right), \tau=\sigma-i \gamma(\sigma \in \mathbb{R}, \gamma>0)$, and $\xi \in \mathbb{R}$.

Remark 2.4.2. Estimate (45)) remains valid with $\tau$ replaced by $\bar{\tau}$.

We define linear sets similar to $D(\mathcal{K})$ and $D_{\beta}(\mathcal{K})$ (see Subsections 1.3 and 1.4). Since the spectrum of the pencil $\mathfrak{B}$ is known, we can describe $D(\mathbb{K})$ and $D_{\beta}(\mathbb{K})$ in more detail. 
Definition 2.4.3. We define the linear set $D(\mathbb{K})$ by the following conditions:

A) If $2 \alpha \leq \pi$, then $D(\mathbb{K})$ is spanned by the functions in $C_{c}^{\infty}(\overline{\mathbb{K}} \backslash O)$ satisfying the boundary conditions (31) and by the functions $\chi v_{s, k}$ corresponding to the eigenvalues of $\mathfrak{B}$ with $\operatorname{Im} \lambda_{k}<1$; here $\chi$ stands for a cut-off function equal to 1 near the vertex.

B) If $2 \alpha \in] \pi, 2 \pi\left[\right.$, then $D(\mathbb{K})$ is spanned by the functions in $C_{c}^{\infty}(\overline{\mathbb{K}} \backslash O)$ satisfying (31), by the functions $\chi v_{s, k}$ corresponding to the eigenvalues of $\mathfrak{B}$ with $\operatorname{Im} \lambda_{k} \leq 0$, and by the linear combinations $\chi\left(\alpha_{s} v_{s, 1}+\beta_{s} v_{s,-1}\right)$, where $\alpha_{s}, \beta_{s}(s=1,2)$ are fixed coefficients such that $\operatorname{Re} \alpha_{s} \overline{\beta_{s}}=0$ and $\left|\alpha_{s}\right|+\left|\beta_{s}\right|>0$.

C) If $2 \alpha=2 \pi$, then $D(\mathbb{K})$ is spanned by the functions in $C_{c}^{\infty}(\overline{\mathbb{K}} \backslash O)$ satisfying (31), by the functions $\chi v_{s, k}$ corresponding to the eigenvalues of $\mathfrak{B}$ with $\operatorname{Im} \lambda_{k} \leq 0$, and by the linear combinations $\chi\left(\alpha_{s} v_{s,+0}+\beta_{s} v_{s,-0}\right)$, where $\alpha_{s}, \beta_{s}(s=1,2)$ are fixed coefficients satisfying $\operatorname{Re} \alpha_{s} \overline{\beta_{s}}=0$ and $\left|\alpha_{s}\right|+\left|\beta_{s}\right|>0$.

The next statement is similar to Theorem 1.3.5.

Proposition 2.4.4. Estimate (45) is valid for any function in $D(\mathbb{K})$.

Now we introduce the linear set $D_{\beta}(\mathbb{K})$ and prove a combined estimate in $\mathbb{K}$.

Definition 2.4.5. Let $\beta<\min \{1, \pi / 2 \alpha\}$. The set $D_{\beta}(\mathbb{K})$ is spanned by the functions in $C_{c}^{\infty}(\overline{\mathbb{K}} \backslash O)$ satisfying the boundary conditions (31) and by the functions $\chi v_{s, k}$ corresponding to the eigenvalues $\lambda$ of $\mathfrak{B}$ with $\operatorname{Im} \lambda<\beta$.

Proposition 2.4.6. For $\beta<\min \{1, \pi / 2 \alpha\}$, suppose that the line $\operatorname{Im} \lambda=\beta$ is free from the spectrum of $\mathfrak{B}$. Then the inequality

$$
\begin{aligned}
\gamma^{2} \| v ; & H_{\beta}^{0}(\mathbb{K})\left\|^{2}+\right\| \chi_{|\tau|} v ; H_{\beta}^{1}(\mathbb{K}, p) \|^{2} \\
& \leq c\left\{\left\|f ; H_{\beta}^{0}(\mathbb{K})\right\|^{2}+\left(|\tau|^{2(1-\beta)} / \gamma^{2}\right)\left\|f ; L_{2}(\mathbb{K})\right\|^{2}\right\}
\end{aligned}
$$

is fulfilled for any $v \in D_{\beta}(\mathbb{K})$, where $f=\left(\tau+A\left(D_{x}, D_{y}, \xi\right)\right) v, \chi_{\tau}(r)=\chi(|\tau| r)$, and $\chi$ is a cut-off function in $C_{c}^{\infty}(\overline{\mathbb{K}})$ equal to 1 near the vertex. The constant $c$ is independent of $\xi$ and $\tau$.

Proof. Step 1. Estimation in a neighborhood of the edge. Applying the Fourier transform $\mathcal{F}_{t \rightarrow \tau}$ to problem (11), (2) in the cylinder $\mathbf{T}$, we arrive at a problem in the wedge $\mathbb{D}$,

$$
\left\{\begin{array}{rlrl}
\tau \widehat{\mathcal{U}}+A\left(D_{x}, D_{y}, D_{z}\right) \widehat{\mathcal{U}} & =\widehat{\mathcal{F}}, & (x, y, z) & \in \mathbb{D}, \\
\Gamma \widehat{\mathcal{U}} & =0, \quad(x, y, z) \in \partial \mathbb{D} \backslash M,
\end{array}\right.
$$

with parameter $\tau$. The new variables

$$
\zeta=|\tau|(x, y, z), \quad U(\zeta, \tau)=\widehat{\mathcal{U}}(\zeta /|\tau|, \tau), \quad F(\zeta, \tau)=|\tau|^{-1} \widehat{\mathcal{F}}(\zeta /|\tau|, \tau)
$$

allow us to rewrite this problem in the form

$$
\left\{\begin{array}{rlrl}
(\tau /|\tau|) U+A\left(D_{\zeta}\right) U & =F, & \zeta=\left(\zeta_{1}, \zeta_{2}, \zeta_{3}\right) & \in \mathbb{D}, \\
\Gamma U & =0, & \zeta \in \partial \mathbb{D} \backslash M .
\end{array}\right.
$$

If $\beta<\min \{1, \pi / 2 \alpha\}$ and the line $\operatorname{Im} \lambda=\beta$ contains no eigenvalues of $\mathfrak{B}$, then the operator $\mathcal{A}_{\beta}(\omega)$ is Fredholm with trivial kernel (see the proof of Theorem 2.3.1). We have

$$
\left\|u ; E_{\beta}^{1}(\mathbb{K})\right\| \leq c\left\|\mathcal{A}_{\beta}(\omega) u ; E_{\beta}^{0}(\mathbb{K})\right\|
$$

for any $u \in E_{\beta}^{1}(\mathbb{K})$ satisfying the boundary conditions $\Gamma u=0$. Using Proposition 2.1.1, we show that

$$
\left\|U ; H_{\beta}^{1}(\mathbb{D})\right\| \leq c\left\|A\left(D_{\zeta}\right) U ; H_{\beta}^{0}(\mathbb{D})\right\|
$$


if $U \in H_{\beta}^{1}(\mathbb{D})$ and $\Gamma U=0$. Consequently,

$$
\left\|\chi U ; H_{\beta}^{1}(\mathbb{D}, 1)\right\| \leq c\left\{\left\|\chi A\left(D_{\zeta}\right) U ; H_{\beta}^{0}(\mathbb{D})\right\|+\left\|\psi U ; H_{\beta}^{0}(\mathbb{D})\right\|\right\},
$$

where $\chi=\chi(r)$ and $\psi=\psi(r)$ are smooth cut-off functions equal to 1 near the vertex and such that $\chi \psi=\chi$. We rewrite this inequality as

$$
\left\|\chi U ; H_{\beta}^{1}(\mathbb{D}, 1)\right\| \leq c\left\{\left\|\chi M\left(D_{\zeta}, \tau /|\tau|\right) U ; H_{\beta}^{0}(\mathbb{D})\right\|+\left\|\psi U ; H_{\beta}^{0}(\mathbb{D})\right\|\right\} .
$$

Step 2. Estimating far from the edge. We prove that, for each $\beta \in \mathbb{R}$ and each $U \in$ $H_{\beta}^{1}(\mathbb{D}, 1)$ satisfying the boundary condition $\Gamma U=0$, the inequality

$$
\text { (48) }(\gamma /|\tau|)^{2}\left\|\kappa_{\infty} U ; H_{\beta}^{0}(\mathbb{D})\right\|^{2} \leq c\left\{\left\|\kappa_{\infty} M\left(D_{\zeta}, \tau /|\tau|\right) U ; H_{\beta}^{0}(\mathbb{D})\right\|^{2}+\left\|\psi_{\infty} U ; H_{\beta-1}^{0}(\mathbb{D})\right\|^{2}\right\}
$$

is valid with a constant $c$ independent of $U$ and $\tau$; here the functions $\kappa_{\infty}=\kappa_{\infty}(r)$ and $\psi_{\infty}=\psi_{\infty}(r)$ are smooth in $\overline{\mathbb{K}}$, vanish near the vertex, and are equal to 1 in a neighborhood of infinity, and $\kappa_{\infty} \psi_{\infty}=\kappa_{\infty}$.

Suppose $\kappa, \psi \in C^{\infty}(\overline{\mathbb{K}}), \kappa \psi=\kappa$, and $\operatorname{supp} \kappa \subset\{(x, y) \in \mathbb{K}: 1 / 2<r<2\}, \operatorname{supp} \psi \subset$ $\{(x, y) \in \mathbb{K}: 1 / 4<r<4\}$. Estimate 45) and Parseval's identity imply that

$$
\gamma^{2}\left\|\kappa U ; L_{2}(\mathbb{D})\right\|^{2} \leq\left\|M\left(D_{\zeta}, \tau\right) \kappa U ; L_{2}(\mathbb{D})\right\|^{2} .
$$

Since $M \kappa U=\kappa M U+[M, \kappa] U$, we have

$$
\gamma^{2}\left\|\kappa U ; L_{2}(\mathbb{D})\right\|^{2} \leq c\left\{\left\|\kappa M\left(D_{\zeta}, \tau\right) U ; L_{2}(\mathbb{D})\right\|^{2}+\left\|\psi U ; L_{2}(\mathbb{D})\right\|^{2}\right\} .
$$

In the role of $U$ we take the function $\zeta \mapsto U^{\varepsilon}(\zeta)=U(\zeta / \varepsilon)$ and replace $\tau$ with $\tau /|\tau| \varepsilon$, where $\varepsilon>0$. Then

$$
(\gamma /|\tau| \varepsilon)^{2}\left\|\kappa U^{\varepsilon} ; L_{2}(\mathbb{D})\right\|^{2} \leq c\left\{\left\|\kappa M\left(D_{\zeta}, \tau /|\tau| \varepsilon\right) U^{\varepsilon}, L_{2}(\mathbb{D})\right\|^{2}+\left\|\psi U^{\varepsilon} ; L_{2}(\mathbb{D})\right\|^{2}\right\} .
$$

After the change of variables $\zeta \mapsto \zeta / \varepsilon$, we obtain

$$
(\gamma /|\tau|)^{2}\left\|\kappa_{\varepsilon} U ; L_{2}(\mathbb{D})\right\|^{2} \leq c\left\{\left\|\kappa_{\varepsilon} M\left(D_{\zeta}, \tau /|\tau|\right) U ; L_{2}(\mathbb{D})\right\|^{2}+\varepsilon^{2}\left\|\psi_{\varepsilon} U ; L_{2}(\mathbb{D})\right\|^{2}\right\},
$$

where $\kappa_{\varepsilon}(\eta)=\kappa(\varepsilon \eta)$. Multiplying this estimate by $\varepsilon^{-2 \beta}$, putting $\varepsilon=2^{-j}, j=1,2,3, \ldots$, and adding the resulting inequalities, we arrive at (48).

Step 3. Estimation in an intermediate zone. As in the proof of Proposition 1.4.2 (see Step 3), from (47) and (48) we deduce the inequality

$$
\begin{aligned}
& \gamma^{2}\left\|u ; H_{\beta}^{0}(\mathbb{D})\right\|^{2}+\left\|\chi_{|\tau|} u ; H_{\beta}^{1}(\mathbb{D},|\tau|)\right\|^{2} \\
& \quad \leq c\left\{\left\|M\left(D_{x}, D_{y}, D_{z}, \tau\right) u ; H_{\beta}^{0}(\mathbb{D})\right\|^{2}+\left(|\tau|^{1-\beta} / \gamma\right)^{2}\left\|M\left(D_{x}, D_{y}, D_{z}, \tau\right) u ; L_{2}(\mathbb{D})\right\|^{2}\right\} .
\end{aligned}
$$

Step 4. Estimation in the angle $\mathbb{K}$. Let $u(x, y, z)=v(x, y) \psi(z)$, where $v \in D_{\beta}(\mathbb{K})$ and $\psi \in C_{0}^{\infty}(\mathbb{R})$. Since $\psi$ is arbitrary, estimate (49) and Parseval's equality lead to (46).

The linear sets $D(\Omega)$ and $D_{\beta}(\Omega)$ are defined like $D(\mathbb{K})$ and $D_{\beta}(\mathbb{K})$.

Proposition 2.4.7. For any $v$ in $D(\Omega)$ we have

$$
\gamma\left\|v ; L_{2}(\Omega)\right\| \leq\left\|M\left(D_{x}, D_{y}, \xi, \tau\right) ; L_{2}(\Omega)\right\| .
$$

Proposition 2.4.8. For $\beta<\min \{1, \pi / 2 \alpha\}$, suppose that the line $\operatorname{Im} \lambda=\beta$ is free from the spectrum of $\mathfrak{B}$. If $\gamma>\gamma_{0}$ with sufficiently large $\gamma_{0}$, then

$$
\begin{aligned}
& \gamma^{2}\left\|v ; H_{\beta}^{0}(\Omega)\right\|^{2}+\left\|\chi_{|\tau|} v ; H_{\beta}^{1}(\Omega, p)\right\|^{2} \\
& \quad \leq c\left(\gamma_{0}\right)\left\{\left\|f ; H_{\beta}^{0}(\Omega)\right\|^{2}+\left(|\tau|^{2(1-\beta)} / \gamma^{2}\right)\left\|f ; L_{2}(\Omega)\right\|^{2}\right\}
\end{aligned}
$$

for every $v$ in $D_{\beta}(\Omega)$, where $f=\left(\tau+A\left(D_{x}, D_{y}, \xi\right)\right) v, \chi_{|\tau|}(r)=\chi(|\tau| r)$, and $\chi$ is any fixed cut-off function in $C_{c}^{\infty}(\bar{\Omega})$ equal to 1 near the point $O$. The constant $c$ is independent of $(\xi, \tau)$ and $v$. 
We introduce the spaces $\mathcal{D} H_{\beta}(\mathbb{K}, \xi, \tau)$ and $\mathcal{R} H_{\beta}(\mathbb{K}, \xi, \tau)$ by completing $C_{0}^{\infty}(\overline{\mathbb{K}} \backslash 0)$ with respect to the norms

$$
\begin{aligned}
\left\|v ; \mathcal{D} H_{\beta}(\mathbb{K}, \xi, \tau)\right\| & =\left(\gamma^{2}\left\|v ; H_{\beta}^{0}(\mathbb{K})\right\|^{2}+\left\|\chi_{|\tau|} v ; H_{\beta}^{1}(\mathbb{K}, p)\right\|^{2}\right)^{1 / 2}, \\
\left\|f ; \mathcal{R} H_{\beta}(\mathbb{K}, \xi, \tau)\right\| & =\left(\left\|f ; H_{\beta}^{0}(\mathbb{K})\right\|^{2}+\left(|\tau|^{1-\beta} / \gamma\right)^{2}\left\|f ; L_{2}(\mathbb{K})\right\|^{2}\right)^{1 / 2} .
\end{aligned}
$$

Replacing $\mathbb{K}$ with $\Omega$, we define $\mathcal{D} H_{\beta}(\Omega, \xi, \tau)$ and $\mathcal{R} H_{\beta}(\Omega, \xi, \tau)$.

2.5. The operators of problems in $\mathbb{K}$ and $\Omega$. With problem (30), (31) in $\mathbb{K}$, we associate the unbounded operator $v \mapsto M(\xi, \tau) v:=M\left(D_{x}, D_{y}, \xi, \tau\right) v$ on $L_{2}(\mathbb{K})$ with domain $D M(\xi, \tau):=D(\mathbb{K})$. Like this was done for the operator $M(\tau)$ in Subsection 1.3, it is easy to show that $M(\xi, \tau)$ admits closure and that the estimate

$$
\gamma\left\|v ; L_{2}(\mathbb{K})\right\| \leq\left\|\overline{M(\xi, \tau)} v ; L_{2}(\mathbb{K})\right\|
$$

is valid for the functions in the domain of the closed operator. In what follows, $M(\xi, \tau)$ and $\operatorname{DM}(\xi, \tau)$ denote the closed operator and its domain. The proof of the next statement is similar to those of Propositions 1.3.6 and 1.3.7.

Proposition 2.5.1. $\operatorname{Ker} M(\xi, \tau)=0, \operatorname{Im} M(\xi, \tau)=L_{2}(\mathbb{K})$.

The operator $A\left(D_{x}, D_{y}, 0\right)$ with domain $D(\mathbb{K})$ is symmetric. From Proposition 2.5.1 it follows that the closure $A$ of $A\left(D_{x}, D_{y}, 0\right)$ is a selfadjoint operator. If $2 \alpha>\pi$, then $D(\mathbb{K})$ contains the combinations $\chi\left(\alpha_{s} v_{s, 1}+\beta_{s} v_{s,-1}\right), s=1$, 2 , where $\alpha_{s}, \beta_{s}$ are fixed coefficients satisfying $\operatorname{Re} \alpha_{s} \bar{\beta}_{s}=0,\left|\alpha_{s}\right|+\left|\beta_{s}\right|>0$. Thus, for $2 \alpha>\pi$, various selfadjoint extensions of $A\left(D_{x}, D_{y}, 0\right)$ can be defined by choosing the parameters $\left\{\alpha_{s}, \beta_{s}\right\}_{s=1,2}$. We turn to the problem in a scale of weighted spaces. For problem (30), (31), we introduce the unbounded operator $v \mapsto M_{\beta}(\xi, \tau):=M\left(D_{x}, D_{y}, \xi, \tau\right)$ with domain $D_{\beta}(\mathbb{K})$ and acting from $\mathcal{D} H_{\beta}(\mathbb{K}, \xi, \tau)$ to $\mathcal{R} H_{\beta}(\mathbb{K}, \xi, \tau)$. The operator $M_{\beta}(\xi, \tau)$ admits closure. We denote by $M_{\beta}(\xi, \tau)$ and $D M_{\beta}(\xi, \tau)$ the closed operator and its domain. Let $1 / 2 \geq \beta_{1}>\cdots$ be all numbers in $]-\infty, 1 / 2]$ such that the line $\operatorname{Im} \lambda=\beta_{k}$ contains an eigenvalue of the pencil $\mathfrak{B}$, and let $S_{m}$ be the total multiplicity of the eigenvalues of $\mathfrak{B}$ in the strip $\operatorname{Im} \lambda \in\left[\beta_{m}, 1 / 2\right]$. If $\lambda=i / 2$ is an eigenvalue of $\mathfrak{B}$, then only half of its multiplicity must be counted in $S_{m}$.

Definition 2.5.2. By a strong solution of problem (30), (31) with right-hand side $f \in$ $L_{2}(\mathbb{K})$ we mean a solution of the equation $M(\xi, \tau) v=f$.

Definition 2.5.3. By a strong $\beta$-solution of problem (30), 313 with right-hand side $f \in \mathcal{R} H_{\beta}(\mathbb{K}, \xi, \tau)$ we mean a solution of the equation $M_{\beta}(\xi, \tau) v=f$.

The next result follows from Proposition 2.5.1.

Theorem 2.5.4. For every $f \in L_{2}(\mathbb{K})$, every $\tau=\sigma-i \gamma(\sigma \in \mathbb{R}, \gamma>0)$, and every $\xi \in \mathbb{R}$, problem (30), (31) with right-hand side $f$ admits a unique strong solution $v$. Moreover,

$$
\gamma\left\|v ; L_{2}(\mathbb{K})\right\| \leq\left\|f ; L_{2}(\mathbb{K})\right\| .
$$

The theorem below can be verified in the same way as Theorem 1.5.3.

Theorem 2.5.5. A) Let $\beta \in] \beta_{1}, \min \{1, \pi / 2 \alpha\}\left[\right.$. For every $f \in \mathcal{R} H_{\beta}(\mathbb{K}, \xi, \tau)$, problem (30), (31) in $\mathbb{K}$ with right-hand side $f$ admits a unique strong $\beta$-solution $v$. Moreover,

$$
\left\|v ; \mathcal{D} H_{\beta}(\mathbb{K}, \xi, \tau)\right\| \leq c\left\|f ; \mathcal{R} H_{\beta}(\mathbb{K}, \xi, \tau)\right\| .
$$

B) Let $\beta \in] \beta_{m+1}, \beta_{m}$ [. A strong $\beta$-solution of problem (301), (31) with right-hand side $f \in \mathcal{R} H_{\beta}(\mathbb{K}, \xi, \tau)$ exists and is unique under the following $S_{m}$ conditions: $\left(f, w_{s, k}\right)_{\mathbb{K}}=0$, where $\left\{w_{s, k}\right\}_{k=-1, \ldots,-m}^{s=1,2}$ is a basis in $\operatorname{Ker} M_{\beta}(\xi, \tau)^{*}$. This solution satisfies the estimate in part $\mathrm{A})$ of the theorem. 
Remark 2.5.6. Theorem 2.5.4 is valid for problem (30), (31) in $\Omega$. Theorem 2.5.5 remains true for problem (30), (31) in $\Omega$ under the supplementary condition $\gamma>\gamma_{0}$ with sufficiently large $\gamma_{0}$.

2.6. The problems in the cylinders $\mathbf{T}$ and $\mathcal{T}$. Applying the Fourier transform $\mathcal{F}_{(\xi, \tau) \rightarrow(z, t)}^{-1}$, we pass to problem (11), (2) in $\mathbf{T}$ (or in $\mathcal{T}$ ).

Definition 2.6.1. Let $f \in V_{0}^{0}(\mathbf{T}, \gamma)$, and let $\widehat{u}(x, y, \xi, \tau)$ be a strong solution of problem (30), (31) in $\mathbb{K}$ with right-hand side $\widehat{f}(x, y, \xi, \tau)=\mathcal{F}_{(z, t) \rightarrow(\xi, \tau)} f(x, y, z, t)$. The function $u$ defined by $u(x, y, z, t)=\mathcal{F}_{(\xi, \tau) \rightarrow(z, t)}^{-1} \widehat{u}(x, y, \xi, \tau)$ is called a strong solution of the problem (1), (2) in $\mathbf{T}$ with right-hand side $f$.

Theorem 2.5.4 leads to the following result.

Theorem 2.6.2. For every $f \in V_{0}^{0}(\mathbf{T}, \gamma)$ and every $\gamma>0$, problem (1), (2) with righthand side $f$ admits a strong solution $v$. Moreover,

$$
\gamma\left\|v ; V_{0}^{0}(\mathbf{T}, \gamma)\right\| \leq\left\|f ; V_{0}^{0}(\mathbf{T}, \gamma)\right\| .
$$

We fix a cut-off function $\chi \in \mathcal{C}^{\infty}(\overline{\mathbb{K}})$ equal to 1 near the corner point $O$ and put

$$
\begin{aligned}
X u(x, y, z, t) & =\mathcal{F}_{\tau \rightarrow t}^{-1} \chi(|\tau| x,|\tau| y) \mathcal{F}_{t^{\prime} \rightarrow \tau} u\left(x, y, z, t^{\prime}\right), \\
\Lambda^{\mu} u(x, y, z, t) & =\mathcal{F}_{\tau \rightarrow t}^{-1}|\tau|^{\mu} \mathcal{F}_{t^{\prime} \rightarrow \tau} u\left(x, y, z, t^{\prime}\right), \\
P^{\mu} u(x, y, z, t) & =\mathcal{F}_{(\xi, \tau) \rightarrow(z, t)}^{-1} p^{\mu} \mathcal{F}_{\left(z^{\prime}, t^{\prime}\right) \rightarrow(\xi, \tau)} u\left(x, y, z^{\prime}, t^{\prime}\right) .
\end{aligned}
$$

Here $\Lambda$ is the same operator as in Subsection 1.7, while $X$ differs by the property that the cut-off function is independent of $z$. We introduce the spaces $\mathcal{D} V_{\beta}(\mathbf{T}, \gamma)$ and $\mathcal{R} V_{\beta}(\mathbf{T}, \gamma)$ with the norms

$$
\begin{aligned}
\left\|u ; \mathcal{D} V_{\beta}(\mathbf{T}, \gamma)\right\| & =\left(\gamma^{2}\left\|u ; V_{\beta}^{0}(\mathbf{T}, \gamma)\right\|^{2}+\left\|X u ; V_{\beta}^{1}(\mathbf{T}, \gamma)\right\|^{2}\right)^{1 / 2} \\
\left\|f ; \mathcal{R} V_{\beta}(\mathbf{T}, \gamma)\right\| & =\left(\left\|f ; V_{\beta}^{0}(\mathbf{T}, \gamma)\right\|^{2}+\left(1 / \gamma^{2}\right)\left\|\Lambda^{1-\beta} f ; V_{0}^{0}(\mathbf{T}, \gamma)\right\|^{2}\right)^{1 / 2}
\end{aligned}
$$

Definition 2.6.3. For $f \in \mathcal{R} V_{\beta}(\mathbf{T}, \gamma)$, let $\widehat{u}(x, y, \xi, \tau)$ be a strong $\beta$-solution of the problem (30), (31) in $\mathbb{K}$ with right-hand side $\widehat{f}(x, y, \xi, \tau)=\mathcal{F}_{(z, t) \rightarrow(\xi, \tau)} f(x, y, z, t)$. The function $u$ given by $u(x, y, z, t)=\mathcal{F}_{(\xi, \tau) \rightarrow(z, t)}^{-1} \widehat{u}(x, y, \xi, \tau)$ is called a strong $\beta$-solution of problem (11), (2) in $\mathbf{T}$ with right-hand side $f$.

The following statement is a consequence of Theorem 2.5.5.

Theorem 2.6.4. Suppose $\alpha<\pi$.

A) Let $\beta \in] \beta_{1}, \min \{1, \pi / 2 \alpha\}\left[\right.$. For every function $f \in \mathcal{R} V_{\beta}(\mathbf{T}, \gamma)$, problem (1), (2) with right-hand side $f$ admits a unique strong $\beta$-solution $v$. Moreover,

$$
\left\|v ; \mathcal{D} V_{\beta}(\mathbf{T}, \gamma)\right\| \leq c\left\|f ; \mathcal{R} V_{\beta}(\mathbf{T}, \gamma)\right\| .
$$

B) Let $\beta \in] \beta_{m+1}, \beta_{m}[$. A strong $\beta$-solution to problem (11), (2) with right-hand side $f \in \mathcal{R} V_{\beta}(\mathbf{T}, \gamma)$ exists and is unique under the following $S_{m}$ conditions:

$$
\left(\widehat{f}(\cdot, \xi, \tau), w_{s, k}(\cdot, \xi, \bar{\tau})\right)_{\mathbb{K}}=0 \quad \text { for all } \xi \in \mathbb{R} \text { and all } \tau \in \mathbb{R}-i \gamma,
$$

where $\left\{w_{s, k}\right\}_{k=-1, \ldots,-m}^{s=1,2}$ is a basis in $\operatorname{Ker} M_{\beta}(\xi, \tau)^{*}$. Such a solution satisfies the estimate in part $\mathrm{A})$ of the theorem.

We turn to the asymptotics of solutions near the edge; the fact to be stated is similar to Theorem 1.7.5. The proof of it can be obtained by an obvious modification of the 
proof of Theorem 1.6.1 and by applying the Fourier transform $\mathcal{F}_{(\xi, \tau) \rightarrow(z, t)}^{-1}$. We introduce the formal series

$$
R_{s, k}(r, \varphi, \xi, \tau)=\sum_{q=0}^{\infty} r^{i \lambda_{k}+q}(M(0,0, \xi, \tau))^{q} \Psi_{q}(\varphi),
$$

where $\Psi_{0}=\Phi_{s, k}$. The series $R_{s, k}$ solves the homogeneous problem (30), (3I). Let $R_{s, k, T}$ denote the partial sum consisting of the first $T$ terms of $R_{s, k}$.

Theorem 2.6.5. Assume that $f$ and $\Lambda^{1-\beta} P^{\beta} f$ are in $\mathcal{R} V_{\beta}(\mathbf{T}, \gamma)$ with $\left.\beta \in\right] \beta_{m+1}, \beta_{m}[$. Then the strong solution of problem (1), (2) in $T$ admits the asymptotic representation

$$
u(x, y, z, t)=\sum R_{s, k, T}\left(r, \varphi, D_{z}, D_{t}\right)\left(X \check{c}_{s, k}\right)(x, y, z, t)+w(x, y, z, t),
$$

where $w \in \mathcal{D} V_{\beta}(\mathbf{T}, \gamma)$. The sum consists of the terms corresponding to the eigenvalues of $\mathfrak{B}$ in the strip $\operatorname{Im} \lambda \in\left[\beta_{m}, \beta_{1}\right]$. The coefficients are given by the formulas

$$
\check{c}_{s, k}(z, t)=\mathcal{F}_{(\xi, \tau) \rightarrow(z, t)}^{-1} c_{s, k}(\xi, \tau) \quad \text { and } \quad c_{s, k}=i\left(\widehat{f}(\cdot, \xi, \tau), w_{s, k}(\cdot, \xi, \bar{\tau})\right)_{\mathbb{K}},
$$

where the collection $\left\{w_{s, k}\right\}_{k=-1, \ldots,-m}^{s=1,2}$ is a basis in $\operatorname{Ker} M_{\beta}(\xi, \tau)^{*}$, as before. Moreover,

$$
\begin{aligned}
\left\|e^{-\gamma t} \check{c}_{s, k}(\cdot) ; H^{\operatorname{Im} \lambda_{k}-\beta}\left(\mathbb{R}^{2}\right)\right\| & \leq c\left\|f ; \mathcal{R} V_{\beta}(\mathbf{T}, \gamma)\right\| \\
\left\|w ; \mathcal{D} V_{\beta}(\mathbf{T}, \gamma)\right\| & \leq(c / \gamma)\left\|\Lambda^{1-\beta} P^{\beta} f ; \mathcal{R} V_{\beta}(\mathbf{T}, \gamma)\right\| .
\end{aligned}
$$

Remark 2.6.6. Theorem 2.6.2 is valid for problem (11), (2) in $\mathcal{T}$. Theorems 2.6 .4 and 2.6.5 remain true for problem (1), (2) in $\mathcal{T}$ under the condition $\gamma>\gamma_{0}$ with sufficiently large $\gamma_{0}$.

2.7. Explicit formulas for the coefficients in the asymptotics of solutions of the problem in $\mathbf{T}$. In Theorem 2.6.5, the coefficients were expressed in terms of a basis $\left\{w_{s, k}\right\}_{k=-1, \ldots,-m}^{s=1,2}$ in the kernel of $M_{\beta}(\xi, \tau)^{*}$. In this subsection, we calculate the elements of that basis explicitly. Note that these elements satisfy the equation $M\left(D_{x}, D_{y}, \xi, \bar{\tau}\right) w_{s, k}=0$ and the boundary conditions (31). Near the vertex of $\mathbb{K}$, the functions $w_{s, k}$ admit the asymptotic representation

$$
w_{s, k}=r^{i \lambda_{-k}} \Phi_{s,-k}(\varphi)+o\left(r^{i \lambda_{-k}}\right) .
$$

Note that if $\alpha=\pi$, then the line $\operatorname{Im} \lambda=\beta_{k}$ contains the eigenvalue $\lambda_{-k+1}$, and we have

$$
w_{s, k}=r^{i \lambda_{-k+1}} \Phi_{s,-k+1}(\varphi)+o\left(r^{i \lambda_{-k+1}}\right) .
$$

From (48) it follows that far from the vertex, the $w_{s, k}$ decay faster than any power of the distance. Since

$$
c_{s, k}(\xi, \tau)=i \int_{\mathbb{K}}\left\langle\widehat{f}(x, y, \xi, \tau), w_{s, k}(x, y, \xi, \tau)\right\rangle_{\mathbb{C}^{8}} d x d y,
$$

we need to find $h_{s, k}=\overline{w_{s, k}}$. Clearly, we have $M\left(D_{x}, D_{y},-\xi,-\tau\right) h_{s, k}=0$ because $M\left(D_{x}, D_{y}, \xi, \bar{\tau}\right) w_{s, k}=0$. In the polar coordinates,

$$
M=\tau I+\xi G+A_{1}(\varphi) D_{r}+(1 / r) A_{2}(\varphi) D_{\varphi}
$$

We put

$$
\widetilde{h}_{s, k}(r, \varphi)=\left(i \tau \eta(r) I-i G \xi \eta(r)+A_{1}(\varphi) \eta^{\prime}(r)\right) r^{i \lambda_{-k}} \Phi_{s,-k}(\varphi),
$$

where $\eta$ is a scalar function. Such a representation of $\widetilde{h}_{s, k}$ is motivated by the corresponding argument for the Helmholtz equation (see [4]) and by the fact that $M\left(D_{x}, D_{y}, \xi, \tau\right)$. $M\left(-D_{x},-D_{y},-\xi, \tau\right)=\Delta+\tau^{2}-\xi^{2}$. We denote $i \tau \eta I-i \xi \eta G+A_{1}(\varphi) \eta^{\prime}$ by $S(r, \varphi, \xi, \tau)$. Then the equation $M\left(D_{x}, D_{y},-\xi,-\tau\right) \widetilde{h}_{s, k}=0$ can be written in the form

$$
\left(-\tau-\xi G+A_{1}(\varphi) D_{r}+(1 / r) A_{2}(\varphi) D_{\varphi}\right) r^{i \lambda_{-k}} S(r, \varphi, \xi, \tau) \Phi_{s,-k}(\varphi)=0 .
$$


Since the eigenfunctions $\Phi_{s,-k}$ of the pencil $\mathfrak{B}$ satisfy equation (35), we have

$$
\frac{d \Phi_{s,-k}(\varphi)}{d \varphi}=-i \lambda_{-k} A_{2}(\varphi) A_{1}(\varphi) \Phi_{s,-k}(\varphi)
$$

Now we transform (51), and write $\lambda$ and $\Phi$ for $\lambda_{-k}$ and $\Phi_{s,-k}$ to simplify the notation:

$$
\begin{gathered}
-(i \tau+i \xi G) r^{i \lambda} S \Phi+i \lambda r^{i \lambda-1} A_{1} S \Phi+r^{i \lambda} A_{1}(d S / d r) \Phi \\
+r^{i \lambda-1} A_{2}(d S / d \varphi) \Phi+r^{i \lambda} S(d \Phi / d \varphi)=0 .
\end{gathered}
$$

Dividing by $r^{i \lambda}$ and using (52), we obtain

(53) $(-i \tau-i \xi G) S+(i \lambda / r) A_{1} S+A_{1}(d S / d r)+(1 / r) A_{2}(d S / d \varphi)-(i \lambda / r) A_{2} S A_{2} A_{1}=0$.

Substituting $S$ and its derivatives in (53) and recalling (34), we see that

$$
\eta^{\prime \prime}+\frac{2 i \lambda_{-k}+1}{r} \eta^{\prime}+\left(\tau^{2}-\xi^{2}\right) \eta=0,
$$

which reduces to Bessel's equation. As a solution, we take $\eta(r)=C r^{\nu} K_{\nu}\left(i \sqrt{\tau^{2}-\xi^{2}} r\right)$, $\nu=i \lambda_{-k}$ (the branch of the square root is chosen in such a way that $\eta(r) \rightarrow 0$ as $r \rightarrow+\infty)$. As $r \rightarrow 0$, we have asymptotic representations

$$
\eta(r)=\frac{C \pi 2^{\nu-1}}{i^{\nu}\left(\tau^{2}-\xi^{2}\right)^{\nu / 2} \sin (\pi \nu) \Gamma(1-\nu)}+o(1), \quad \eta^{\prime}(r)=o(1) .
$$

Since $G \Phi_{1,-k}=\Phi_{2,-k}$ (see Proposition 2.2.1), we obtain

$$
\begin{aligned}
& \widetilde{h}_{1, k}=\eta(0)\left(i \tau \Phi_{1,-k}(\varphi)-i \xi \Phi_{2,-k}(\varphi)\right) r^{i \lambda_{-k}}+o\left(r i \lambda_{-k}\right), \\
& \widetilde{h}_{2, k}=\eta(0)\left(i \tau \Phi_{2,-k}(\varphi)-i \xi \Phi_{1,-k}(\varphi)\right) r^{i \lambda_{-k}}+o\left(r i \lambda_{-k}\right) .
\end{aligned}
$$

We put

$$
h_{1, k}=\tau \widetilde{h}_{1, k}+\xi \widetilde{h}_{2, k}, \quad h_{2, k}=\tau \widetilde{h}_{2, k}+\xi \widetilde{h}_{1, k} .
$$

Then, in a neighborhood of the vertex,

$$
\begin{aligned}
& h_{1, k}=i \eta(0)\left(\tau^{2}-\xi^{2}\right) r^{i \lambda_{-k}} \Phi_{1,-k}+o\left(r i \lambda_{-k}\right), \\
& h_{2, k}=i \eta(0)\left(\tau^{2}-\xi^{2}\right) r^{i \lambda_{-k}} \Phi_{2,-k}+o\left(r i \lambda_{-k}\right) .
\end{aligned}
$$

We fix the constant $C$ by assuming that $i \eta(0)\left(\tau^{2}-\xi^{2}\right)=1$, i.e., we take

$$
C=\frac{\sin (\pi \nu) \Gamma(1-\nu)}{\pi 2^{\nu-1}} i^{\nu-1}\left(\tau^{2}-\xi^{2}\right)^{(\nu-2) / 2} .
$$

Finally, we have

$$
\begin{aligned}
\eta(r) & =\frac{\sin (\pi \nu) \Gamma(1-\nu)}{i \pi 2^{\nu-1}} \frac{1}{\tau^{2}-\xi^{2}}\left(i r \sqrt{\tau^{2}-\xi^{2}}\right)^{\nu} K_{\nu}\left(i r \sqrt{\tau^{2}-\xi^{2}}\right), \\
\eta^{\prime}(r) & =\frac{\sin (\pi \nu) \Gamma(1-\nu)}{i \pi 2^{\nu-1}} r\left(i r \sqrt{\tau^{2}-\xi^{2}}\right)^{\nu-1} K_{\nu-1}\left(i r \sqrt{\tau^{2}-\xi^{2}}\right) .
\end{aligned}
$$

Let $B$ stand for the number $\sin (\pi \nu) \Gamma(1-\nu) /\left(\pi 2^{\nu-1}\right)$. We recall that

$$
\begin{aligned}
& h_{1, k}=i\left(\tau^{2}-\xi^{2}\right) \eta(r) r^{i \lambda_{-k}} \Phi_{1,-k}+\tau A_{1} \eta^{\prime}(r) r^{i \lambda_{-k}} \Phi_{1,-k}+\xi A_{1} \eta^{\prime}(r) r^{i \lambda_{-k}} \Phi_{2,-k}, \\
& h_{2, k}=i\left(\tau^{2}-\xi^{2}\right) \eta(r) r^{i \lambda_{-k}} \Phi_{2,-k}+\xi A_{1} \eta^{\prime}(r) r^{i \lambda_{-k}} \Phi_{1,-k}+\tau A_{1} \eta^{\prime}(r) r^{i \lambda_{-k}} \Phi_{2,-k} .
\end{aligned}
$$

In order to find $W_{s, k}=\mathcal{F}_{(\xi, \tau) \rightarrow(z, t)}^{-1} h_{s, k}$, we need to calculate the Fourier transform $\mathcal{F}_{(\xi, \tau) \rightarrow(z, t)}^{-1}$ of the functions

$$
\left(i r \sqrt{\tau^{2}-\xi^{2}}\right)^{\nu} K_{\nu}\left(i r \sqrt{\tau^{2}-\xi^{2}}\right), \quad\left(i r \sqrt{\tau^{2}-\xi^{2}}\right)^{\nu-1} K_{\nu-1}\left(i r \sqrt{\tau^{2}-\xi^{2}}\right) .
$$


Denoting these functions by $f_{\nu}(r, z, t)$ and $f_{\nu-1}(r, z, t)$, respectively, we have

$$
\begin{aligned}
W_{1, k}(x, y, z, t)= & B f_{\nu}(r, z, t) r^{i \lambda_{-k}} \Phi_{1,-k}-B A_{1}(\varphi) \frac{\partial f_{\nu-1}}{\partial t} r^{i \lambda_{-k}} \Phi_{1,-k} \\
& -B A_{1}(\varphi) \frac{\partial f_{\nu-1}}{\partial z} r^{i \lambda_{-k}} \Phi_{2,-k}, \\
W_{2, k}(x, y, z, t)= & B f_{\nu}(r, z, t) r^{i \lambda_{-k}} \Phi_{2,-k}-B A_{1}(\varphi) \frac{\partial f_{\nu-1}}{\partial t} r^{i \lambda_{-k}} \Phi_{2,-k} \\
& -B A_{1}(\varphi) \frac{\partial f_{\nu-1}}{\partial z} r^{i \lambda_{-k}} \Phi_{1,-k} .
\end{aligned}
$$

Now we calculate $f_{p}$ for $p=\nu, \nu-1$ :

$$
f_{p}(r, z, t)=\int_{\mathbb{R}} d \xi \int_{\mathbb{R}-i \gamma} d \tau e^{i z \xi+i t \tau}\left(i r \sqrt{\tau^{2}-\xi^{2}}\right)^{p} K_{p}\left(i r \sqrt{\tau^{2}-\xi^{2}}\right) .
$$

After the change of variables $u=i(\tau+\xi) / 2, s=i(\tau-\xi) / 2$, we obtain

$$
f_{p}(r, z, t)=2 \int_{\operatorname{Re} u=\gamma / 2} d u \int_{\operatorname{Re} s=\gamma / 2} d s e^{u(t+z)+s(t-z)}\left(2 r u^{1 / 2} s^{1 / 2}\right)^{p} K_{p}\left(2 r u^{1 / 2} s^{1 / 2}\right) .
$$

The identity

$$
\int_{\operatorname{Re} s=\gamma / 2} d s e^{s t} s^{\nu / 2} K_{\nu}\left(2 \alpha^{1 / 2} s^{1 / 2}\right)=\frac{1}{2} \theta(t) \alpha^{\nu / 2} e^{-\alpha / t} \frac{1}{t^{\nu+1}}
$$

(see [14]) leads to the relation

$$
f_{p}(r, z, t)=2^{p} r^{2 p} \int_{\operatorname{Re} u=\gamma / 2} d u e^{u(t+z)} u^{p} \theta(t-z) e^{-r^{2} u /(t-z)} \frac{1}{(t-z)^{p+1}} .
$$

We employ the following formulas for the Laplace transform (see [15]):

$$
f_{\alpha}(t)=\int_{\operatorname{Re} p=\gamma / 2} e^{p t} \frac{1}{p^{\alpha}} d p, \quad f_{\alpha}(t)= \begin{cases}\frac{\theta(t)}{\Gamma(\alpha)} t^{\alpha-1}, & \alpha>0, \\ \frac{d^{N} f_{\alpha+N}(t)}{d t^{N}}, & \alpha \leq 0, \alpha+N>0 .\end{cases}
$$

This yields

$$
f_{p}(r, z, t)=\left.2^{p} r^{2 p} \theta(t-z) \frac{1}{(t-z)^{p+1}} \frac{d^{N}}{d v^{N}} f_{N-p}(v)\right|_{v=\left(t^{2}-z^{2}-r^{2}\right) /(t-z)}
$$

where $p=\nu, \nu-1(\nu>0)$. Thus, we have obtained explicit expressions for the functions $h_{s, k}$ and $W_{s, k}$ involved in the following representations of the coefficients in the asymptotic formula (50):

$$
\begin{aligned}
c_{s, k}(\xi, \tau) & =\int_{\mathbb{K}}\left\langle\widehat{f}(x, y, \xi, \tau), h_{s, k}(x, y, \xi, \tau)\right\rangle_{\mathbb{R}^{8}} d x d y, \\
\check{c}_{s, k}(z, t) & =\int_{\mathbb{K}} \int_{\mathbb{R}} \int_{\mathbb{R}}\left\langle f(x, y, z-s, t-u), W_{s, k}(x, y, s, u)\right\rangle_{\mathbb{R}^{8}} d x d y d s d u .
\end{aligned}
$$

2.8. Relationship between the augmented and nonaugmented Maxwell systems. Up to this point, we have dealt with the augmented Maxwell system. Now we are going to prove that, under some conditions on the right-hand side of such a system, its solutions satisfy the usual (nonaugmented) Maxwell system. For sufficiently smooth vector fields $\vec{E}$ and $\vec{B}$, the right-hand side $(-\vec{J},-\vec{G}, \rho, \mu)$ of the usual Maxwell system is subject to the compatibility conditions $\partial \rho / \partial t+\operatorname{div} \vec{J}=0, \partial \mu / \partial t+\operatorname{div} \vec{G}=0$ and the boundary condition $(\vec{G} \cdot \vec{n})=0$. We show that, for a certain selfadjoint extension of $A(\partial)$ taken as the spatial part of the augmented system, the following is true: if for a right-hand side $\left(\overrightarrow{f_{1}}, \vec{f}_{2}, g_{1}, g_{2}\right)$ we have $\partial g_{k} / \partial t-\operatorname{div} \vec{f}_{k}=0(k=1,2)$ inside the domain 
and the boundary condition $\left(\overrightarrow{f_{2}} \cdot \vec{n}\right)=0$ is satisfied, then the components $h$ and $q$ of the corresponding solution $u=(\vec{u}, \vec{v}, h, q)$ vanish identically.

Consider the problem in a bounded domain $G \subset \mathbb{R}^{3}$ with a conical point $O$. After the Fourier transform, the conditions on the right-hand side take the form $i \tau \widehat{g_{k}}-\operatorname{div} \widehat{\overrightarrow{f_{k}}}=0$ $(k=1,2),\left(\widehat{\overrightarrow{f_{2}}} \cdot \vec{n}\right)=0$. Since the right-hand side belongs to $L_{2}(G)$, these conditions must be understood in a proper way. Suppose $\operatorname{div} \widehat{\overrightarrow{f_{1}}}$, div $\widehat{\overrightarrow{f_{2}}} \in L_{2}(G)$, where div is understood in the sense of distributions. The boundary condition $\left(\widehat{\overrightarrow{f_{2}}} \cdot \vec{n}\right)=0$ means that

$$
\left(\widehat{\overrightarrow{f_{2}}}, \nabla \psi\right)_{G}=-\left(\operatorname{div} \widehat{\overrightarrow{f_{2}}}, \psi\right)_{G}, \quad \psi \in H^{1}(G)
$$

The above properties of the vector fields $\widehat{\vec{f}}_{k}$ can be written in a different form. For this, we introduce the space

$$
H(\operatorname{div}, G)=\left\{\vec{u} \in L_{2}(G): \operatorname{div} \vec{u} \in L_{2}(G)\right\}
$$

with the norm $\|\vec{u} ; H(\operatorname{div}, G)\|=\left(\left\|\vec{u} ; L_{2}(G)\right\|^{2}+\left\|\operatorname{div} \vec{u} ; L_{2}(G)\right\|^{2}\right)^{1 / 2}$, and its closed subspace

$$
\stackrel{\circ}{\mathrm{H}}(\operatorname{div}, G)=\{\vec{u} \in H(\operatorname{div}, G):(\vec{u} \cdot \vec{n})=0\} .
$$

Then $\widehat{\overrightarrow{f_{1}}} \in H(\operatorname{div}, G), \widehat{\overrightarrow{f_{2}}} \in \stackrel{\circ}{\mathrm{H}}(\operatorname{div}, G), \widehat{g_{1}}, \widehat{g_{2}} \in L_{2}(G), i \tau \widehat{g_{k}}=\operatorname{div} \widehat{\overrightarrow{f_{k}}}$.

We consider $M(\tau)=\tau+A$, where $A$ is the selfadjoint extension of the differential operator $A\left(D_{x}, D_{y}, D_{z}\right)$ defined on the linear set $D(G)$ for which all the $\alpha_{s, k}$ vanish. In other words, the functions in the domain of $A$ increase near the conical point $O$ more slowly than the functions in the domains of all other selfadjoint extensions.

For the proof of the theorem on relationship between the solutions of the augmented and the usual Maxwell systems, we need two linear sets. Let $\left\{\mu_{k}, w_{k}\right\}$ and $\left\{\widetilde{\mu}_{k}, \widetilde{w}_{k}\right\}$ be the collections of eigenvalues and eigenfunctions of the operator pencils of the Dirichlet and Neumann problems for the Laplace equation in $G$. We denote by $L_{D}$ the linear span of all $C_{c}^{\infty}(G)$-functions and of the functions of the form $\chi r^{i \mu_{k}} w_{k}$ with $\operatorname{Im} \mu_{k}<0$, where $\chi$ is a cut-off function equal to 1 near the conical point. Also, we introduce the linear set $L_{N}$ spanned by the $C_{c}^{\infty}(\bar{G} \backslash O)$-functions with normal derivative vanishing on $\partial G \backslash O$ and by the functions of the form $\chi r^{i \widetilde{\mu}_{k}} \widetilde{w}_{k}$, where $\operatorname{Im} \widetilde{\mu}_{k} \leq 0$. By the results of [1] and [4], the range of the Helmholtz operator $\tau^{2}+\Delta$ with $\tau=\sigma-i \gamma(\gamma \neq 0)$ defined on $L_{D}$ or $L_{N}$ is dense in $L_{2}(G)$.

Theorem 2.8.1. 1) Suppose that the selfadjoint extension A mentioned above is taken as the spatial part of system (5), (6) and that the right-hand side $f=(-\vec{J},-\vec{G}, \rho, \mu)$ of this system is subject to the conditions $\rho, \mu \in L_{2}(G), \vec{J} \in H(\operatorname{div}, G), \vec{G} \in \stackrel{H}{H}(\operatorname{div}, G)$, $i \tau \rho+\operatorname{div} \vec{J}=0$, and $i \tau \mu+\operatorname{div} \vec{G}=0$. Then the corresponding strong solution $u$ is of the form $u=(\vec{u}, \vec{v}, 0,0)$.

2) If the role of the spatial part is played by a selfadjoint extension different from that in 1), then there exist right-hand sides subject to the conditions listed in 1) and such that the corresponding strong solutions have nonzero components $h, q$.

Proof. We prove the first statement. Since $A$ is the closure of the differential operator $A\left(D_{x}, D_{y}, D_{z}\right)$ defined on $D(G)$, there exists a sequence $\left\{u_{k}\right\} \subset D(G)$ such that $u_{k} \rightarrow u$ and $f_{k}:=M(\tau) u_{k} \rightarrow f$ (convergence in $\left.L_{2}(G)\right)$. We have $u_{k} \in C^{\infty}(\bar{G} \backslash O)$ ), so that system (5), (6) can be understood in the usual sense. In particular,

$$
\begin{aligned}
i \tau \vec{u}_{k}-\operatorname{curl} \vec{v}_{k}+\nabla h_{k} & =-\vec{J}_{k}, \\
i \tau h_{k}+\operatorname{div} \vec{u}_{k} & =\rho_{k} ;
\end{aligned}
$$


moreover, $u_{k}$ satisfies the boundary conditions (6) on $\partial G \backslash O$. We show that $h=0$. Let $\phi \in C_{0}^{\infty}(G)$. We multiply the first equation by $\nabla \phi$ and the second by $\phi$, and then integrate over $G$. This yields

$$
\begin{aligned}
& i \tau\left(\vec{u}_{k}, \nabla \phi\right)_{G}-\left(\operatorname{curl} \vec{v}_{k}, \nabla \phi\right)_{G}+\left(\nabla h_{k}, \nabla \phi\right)_{G}=-\left(\vec{J}_{k}, \nabla \phi\right)_{G}, \\
&-\tau^{2}\left(h_{k}, \phi\right)_{G}+i \tau\left(\operatorname{div} \vec{u}_{k}, \phi\right)_{G}=i \tau\left(\rho_{k}, \phi\right)_{G} .
\end{aligned}
$$

In the first relation, we integrate the first two terms by parts. Multiplying the second relation by $i \tau$ and adding the result to the first, we obtain

$$
-\tau^{2}\left(h_{k}, \phi\right)_{G}+\left(\nabla h_{k}, \nabla \phi\right)_{G}=i \tau\left(\rho_{k}, \phi\right)_{G}-\left(\vec{J}_{k}, \nabla \phi\right)_{G} .
$$

Since $L_{D} \subset \stackrel{\circ}{H}^{1}(G)$, for any $\psi$ in $L_{D}$ there exists a sequence $\left\{\phi_{m}\right\} \subset C_{0}^{\infty}(G)$ such that $\phi_{m} \rightarrow \psi, \nabla \phi_{m} \rightarrow \nabla \psi$, and

$$
-\tau^{2}\left(h_{k}, \psi\right)_{G}+\left(\nabla h_{k}, \nabla \psi\right)_{G}=i \tau\left(\rho_{k}, \psi\right)_{G}-\left(\vec{J}_{k}, \nabla \psi\right)_{G} .
$$

We integrate the second term by parts, let $k \rightarrow+\infty$, and recall that $-(\vec{J}, \nabla \psi)_{G}=$ $(\operatorname{div} \vec{J}, \psi)_{G}$ and $\operatorname{div} \vec{J}+i \tau \rho=0$, obtaining

$$
\left(h,\left(\bar{\tau}^{2}+\Delta\right) \psi\right)_{G}=0 .
$$

It follows that $h=0$, because the range of the operator $\bar{\tau}^{2}+\Delta$ defined on $L_{D}$ is dense in $L_{2}(G)$.

Now, we check that $q=0$. We have

$$
\begin{aligned}
i \tau \vec{v}_{k}+\operatorname{curl} \vec{u}_{k}+\nabla q_{k} & =-\vec{G}_{k}, \\
i \tau q_{k}+\operatorname{div} \vec{v}_{k} & =\mu_{k} .
\end{aligned}
$$

For $\phi \in L_{N}$, we can write

$$
\begin{aligned}
i \tau\left(\vec{v}_{k}, \nabla \phi\right)_{G}+ & \left(\operatorname{curl} \vec{u}_{k}, \nabla \phi\right)_{G}+\left(\nabla q_{k}, \nabla \phi\right)_{G}=-\left(\vec{G}_{k}, \nabla \phi\right)_{G}, \\
-\tau^{2}\left(q_{k}, \phi\right)_{G}+i \tau\left(\operatorname{div} \vec{v}_{k}, \phi\right)_{G} & =i \tau\left(\mu_{k}, \phi\right)_{G} .
\end{aligned}
$$

We integrate by parts all terms on the left-hand side of the first relation, add the result to the second relation, and let $k \rightarrow \infty$ :

$$
\left(q,\left(\bar{\tau}^{2}+\Delta\right) \phi\right)_{G}=i \tau(\mu, \phi)_{G}-(\vec{G}, \nabla \phi)_{G} .
$$

Since $\vec{G} \in \stackrel{\circ}{\mathrm{H}}(\operatorname{div}, G), \phi \in L_{N} \subset H^{1}(G)$, and $\operatorname{div} \vec{G}+i \tau \mu=0$, we obtain

$$
\left(q,\left(\bar{\tau}^{2}+\Delta\right) \phi\right)_{G}=0 \text {. }
$$

Since the range of the operator $\bar{\tau}^{2}+\Delta$ defined on $L_{N}$ is dense in $L_{2}(G)$, we conclude that $q=0$.

We turn to the second statement of the theorem. The domain of any other selfadjoint extension contains at least one function $\chi r^{i \lambda_{k}} \Phi_{s, k}$ with $\left.\operatorname{Im} \lambda_{k} \in\right] 1,3 / 2[$. By the properties of the pencil $\mathfrak{A}$ (see Subsection 1.2), the eigenfunction $\Phi_{s, k}$ is of the form $(\vec{U}, \overrightarrow{0}, 0, Q)$ or $(\overrightarrow{0}, \vec{V}, H, 0)$. For instance, assume that $\Phi_{s, k}=(\vec{U}, \overrightarrow{0}, 0, Q)$. In accordance with the results of [4] (in particular, see $\S 4$ therein), the homogeneous Neumann problem for the equation $\Delta q_{0}+\tau^{2} q_{0}=0$ admits a solution $q_{0} \in C^{\infty}(\bar{G} \backslash O)$ with asymptotics $q_{0} \sim r^{i \lambda_{k}} Q$ near the conical point $O$. We set $u_{0}:=\left(\chi r^{i \lambda_{k}} \vec{U}, \overrightarrow{0}, 0, q_{0}\right)$ and $f_{0}:=M(\tau) u_{0}=\left(i \tau \chi r^{i \lambda_{k}} \vec{U}, \operatorname{curl}\left(\chi r^{i \lambda_{k}} \vec{U}\right)+\nabla q_{0}, \operatorname{div}\left(\chi r^{i \lambda_{k}} \vec{U}\right), i \tau q_{0}\right)$. It is not hard to see that $f_{0}$ belongs to $L_{2}(G) \cap C^{\infty}(\bar{G} \backslash O)$ and satisfies the conditions listed in part 1) of the theorem. However, the component $q_{0}$ of the solution $u_{0}$ differs from zero. Now, assume that $\Phi_{s, k}=(\overrightarrow{0}, \vec{V}, H, 0)$. By [1. Proposition 5.2], the homogeneous Dirichlet problem for the equation $\Delta h_{0}+\tau^{2} h_{0}=0$ has a solution $h_{0} \in C^{\infty}(\bar{G} \backslash O)$ with asymptotics $h_{0} \sim r^{i \lambda_{k}} H$. We set $u_{1}=\left(\overrightarrow{0}, \chi r^{i \lambda_{k}} \vec{V}, h_{0}, 0\right)$ and $f_{1}:=M(\tau) u_{1}=$ 
$\left(-\operatorname{curl}\left(\chi r^{i \lambda_{k}} \vec{V}\right)+\nabla h_{0}, i \tau \chi r^{i \lambda_{k}} \vec{V}, i \tau h_{0}, \operatorname{div}\left(\chi r^{i \lambda_{k}} \vec{V}\right)\right)$. The function $f_{1}$ satisfies the conditions in 1). However, the component $h_{1}$ of $u_{1}$ is nonzero.

The extension $A$ chosen in part 1 of Theorem 2.8.1 coincides with the operator investigated in [7]. Before proving this, we recall some definitions and statements presented in [7; we take into account that, in our case, the dielectric and magnetic permittivity matrices are equal to the identity matrix. Set

$$
F(G)=\left\{\vec{u} \in L_{2}(G): \operatorname{div} \vec{u} \in L_{2}(G), \operatorname{curl} \vec{u} \in L_{2}(G)\right\} .
$$

The class $F(G)$ is a complete Hilbert space with inner product defined by the norm

$$
\|\vec{u} ; F(G)\|=\left(\left\|\operatorname{div} \vec{u} ; L_{2}(G)\right\|^{2}+\left\|\operatorname{curl} \vec{u} ; L_{2}(G)\right\|^{2}+\left\|\vec{u} ; L_{2}(G)\right\|^{2}\right)^{1 / 2} .
$$

We introduce the closed subspaces

$$
F(\tau, G)=\{\vec{u} \in F(G): \vec{u} \times \vec{n}=0\}, \quad F(\nu, G)=\{\vec{u} \in F(G): \vec{u} \cdot \vec{n}=0\} .
$$

The condition $\vec{u} \times \vec{n}=0$ is understood in the following sense:

$$
(\vec{u}, \operatorname{curl} \vec{z})_{G}=(\operatorname{curl} \vec{u}, \vec{z})_{G} \quad \text { for all } \vec{z} \in L_{2}(G) \text { with } \operatorname{curl} \vec{z} \in L_{2}(G) \text {. }
$$

The dense subsets $D(\nu, G)=F(\nu, G) \oplus \stackrel{\circ}{\mathrm{H}}^{1}(G)$ and $D(\tau, G)=F(\tau, G) \oplus H^{1}(G)$ in the Hilbert space $B(G)=L_{2}\left(G, \mathbb{C}^{3}\right) \oplus L_{2}(G)$ are taken as the domains of the operators $L(\nu)\{\vec{v}, q\}=\{\operatorname{curl} \vec{v}+\nabla q,-\operatorname{div} \vec{v}\}$ and $L(\tau)\{\vec{u}, h\}=\{\operatorname{curl} \vec{u}+\nabla h,-\operatorname{div} \vec{u}\}$. It turns out (see [7, Subsection 2.4]) that the block operator

$$
\mathcal{L}=\left(\begin{array}{cc}
0 & i L(\nu) \\
-i L(\tau) & 0
\end{array}\right)
$$

is selfadjoint in $B(G) \oplus B(G)$. Let $P$ be the matrix defined by $P(\vec{u}, \vec{v}, h, q)^{T}=(\vec{u},-q, \vec{v}, h)^{T}$. We prove that $P A P^{-1}=\mathcal{L}$. The properties of the pencil $\mathfrak{A}$ and the asymptotics of the functions in $D(G)$ near the conical point $O$ imply that $P(D(G)) \subset D(\nu, G) \oplus D(\tau, G)$. The selfadjoint operator $A$ is the closure of the differential operator $A\left(D_{x}, D_{y}, D_{z}\right)$ defined on $D(G)$. Since the operators $A\left(D_{x}, D_{y}, D_{z}\right)$ and $P^{-1} A P$ coincide on $D(G)$, we obtain $A=P^{-1} \mathcal{L} P$.

Turning to the problem in the wedge $\mathbb{D}$, we recall that the definition of a strong solution of problem (1), (2) in $\mathbf{T}=\mathbb{D} \times \mathbb{R}$ was given in Subsection 2.6.1. Let $D(\mathbb{D})$ be the linear set spanned by the functions in $D(\mathbb{K})$ with coefficients in the Schwartz class $S\left(\mathbb{R}_{z}\right)$, and let $A$ denote the closure in $L_{2}(\mathbb{D})$ of the differential operator $A\left(D_{x}, D_{y}, D_{z}\right)$ defined on $D(\mathbb{K})$. It is easily seen that $A$ is a selfadjoint operator. A strong solution of the problem (1), (2) in $\mathbf{T}$ with right-hand side $f \in V_{0}^{0}(\mathbf{T}, \gamma)$ can be defined by the formula $u=\mathcal{F}_{\tau \rightarrow t}^{-1}(\tau+A)^{-1} \mathcal{F}_{t \rightarrow \tau} f$. By a strong solution of problem (5), (6) in $\mathbb{D}$ with right-hand side $f \in L_{2}(\mathbb{D})$ we mean a solution of the equation $(\tau+A) u=f$. For $2 \alpha>\pi$, various operators $A$ can be obtained by fixing the choice of the constants $\alpha_{s}, \beta_{s}$ for $D(\mathbb{K})$ (see Definition 2.4.3). We take the operator $A$ corresponding to $\alpha_{s}=0, s=1,2$. The next statement is similar to Theorem 2.8.1.

Theorem 2.8.2. 1) Suppose the operator A mentioned above is chosen to be the spatial part of system (5), (ㅁ) in $\mathbb{D}$. Let $f=(-\vec{J},-\vec{G}, \rho, \mu)$ be the right-hand side of the system, where $\rho, \mu \in L_{2}(\mathbb{D}), \vec{J} \in H(\operatorname{div}, \mathbb{D}), \vec{G} \in \stackrel{\circ}{H}(\operatorname{div}, \mathbb{D})$, and $i \tau \rho+\operatorname{div} \vec{J}=0, i \tau \mu+\operatorname{div} \vec{G}=0$. Then the strong solution is of the form $u=(\vec{u}, \vec{v}, 0,0)$.

2) Suppose a selfadjoint extension A different from that in 1) plays the role of the spatial part. Then there exist right-hand sides satisfying the conditions in 1) and such that the components $h, q$ of the corresponding strong solutions are nonzero. 
2.9. The Maxwell system with impedance boundary conditions. We consider the Maxwell system in a bounded domain $G \subset \mathbb{R}^{3}$,

$$
\left\{\begin{array}{c}
\partial \vec{E} / \partial t-\operatorname{curl} \vec{B}=-\vec{J}, \\
\partial \vec{B} / \partial t+\operatorname{curl} \vec{E}=0, \\
\operatorname{div} \vec{E}=\rho, \\
\operatorname{div} \vec{B}=0,
\end{array}\right.
$$

with impedance boundary conditions on $\partial G$ :

$$
\vec{n} \times[\vec{B} \times \vec{n}]+\psi[\vec{n} \times \vec{E}]=[\vec{\Phi} \times \vec{n}] .
$$

Here $\psi$ denotes a complex function on $\partial G$ satisfying $\operatorname{Re} \psi<0$. For simplicity, we assume that $\psi=a+i b, a<0$, where $a, b$ are real constants. To employ the same method as before, we introduce the augmented system

$$
\left\{\begin{array}{c}
\partial \vec{u} / \partial t-\operatorname{curl} \vec{v}+\nabla h=\vec{A}_{1}, \\
\partial \vec{v} / \partial t+\operatorname{curl} \vec{u}+\nabla q=\vec{A}_{2}, \\
\partial h / \partial t+\operatorname{div} \vec{u}=g_{1}, \\
\partial q / \partial t+\operatorname{div} \vec{v}=g_{2} ;
\end{array}\right.
$$

the spatial part here will be denoted by $A(\partial)$. The corresponding "augmented" impedance conditions are of the form

$$
\vec{n} \times[\vec{v} \times \vec{n}]+\psi[\vec{n} \times \vec{u}]=[\vec{\Phi} \times \vec{n}], \quad h=H, \quad q=Q .
$$

We list some properties of the augmented Maxwell system with impedance boundary conditions without proofs.

Proposition 2.9.1. 1) The Green formula holds,

$$
\begin{aligned}
\int_{V}\left\langle A(\partial) \mathcal{U}_{1}, \mathcal{U}_{2}\right\rangle d V+\int_{V}\left\langle\mathcal{U}_{1}, A(\partial) \mathcal{U}_{2}\right\rangle d V \\
=\int_{\partial V}\left\langle\Gamma_{1} \mathcal{U}_{1}, T_{1} \mathcal{U}_{2}\right\rangle d S+\int_{\partial V}\left\langle T_{2} \mathcal{U}_{1}, \Gamma_{2} \mathcal{U}_{2}\right\rangle d S
\end{aligned}
$$

where $V \subset \mathbb{R}^{3}$ is a domain with smooth boundary, $\mathcal{U}_{1}, \mathcal{U}_{2} \in \mathcal{C}^{\infty}(\bar{V})$, and, for $\mathcal{U}=$ $(\vec{u}, \vec{v}, h, q)$, we put $\Gamma_{1} \mathcal{U}=(\vec{n} \times[\vec{v} \times \vec{n}]+\psi[\vec{n} \times \vec{u}], h, q)^{T}, T_{1} \mathcal{U}=((1 / \bar{\psi}) \vec{n} \times[\vec{v} \times \vec{n}]$, $(\vec{n} \cdot \vec{u}),(\vec{n} \cdot \vec{v}))^{T}, \Gamma_{2} \mathcal{U}=(-\vec{n} \times[\vec{v} \times \vec{n}]+\bar{\psi}[\vec{n} \times \vec{u}], h, q)^{T}$, and $T_{2} \mathcal{U}=((1 / \psi) \vec{n} \times[\vec{v} \times \vec{n}],(\vec{n} \cdot \vec{u})$, $(\vec{n} \cdot \vec{v}))^{T}(\vec{n}$ denotes the unit outward normal to $\partial V)$.

2) The problems $\left\{A(\partial), \Gamma_{1}\right\}$ and $\left\{A(\partial), \Gamma_{2}\right\}$ are adjoint to each other with respect to the Green formula (55) and are elliptic.

We prove a global energy estimate for the augmented Maxwell system with impedance conditions.

Proposition 2.9.2. Suppose $v=(\vec{u}, \vec{v}, h, q) \in C_{c}^{\infty}(\bar{G} \backslash O)$ satisfies (54) with $Q=0$, $H=0$. Then

$$
\begin{aligned}
& \gamma^{2}\left\|v ; L_{2}(G)\right\|^{2}+(\gamma|a| /|\psi|) \cdot\left\|\vec{v} \times \vec{n} ; L_{2}(\partial G)\right\|^{2} \\
& \quad \leq c\left\{\left\|M\left(D_{x}, D_{y}, D_{z}, \tau\right) v ; L_{2}(G)\right\|^{2}+(\gamma|\psi| /|a|) \cdot\left\|\vec{\Phi} \times \vec{n} ; L_{2}(\partial G)\right\|^{2}\right\},
\end{aligned}
$$

where $M\left(D_{x}, D_{y}, D_{z}, \tau\right)=\tau+A(D)$, and the constant $c$ is independent of $v$ and $\tau$. 
Proof. Let $u(x, y, z, t)=\zeta(t) v(x, y, z)$ with $\zeta \in C_{0}^{\infty}(\mathbb{R})$. From (55) it follows that

$$
\begin{aligned}
& \operatorname{Re} \int_{G}\langle A(\partial) u, u\rangle d x d y d z \\
& \quad=\operatorname{Re} \int_{\partial G}(\vec{u} \cdot[\vec{v} \times \vec{n}]) d S=\operatorname{Re} \frac{1}{\psi} \int_{\partial G}\left([\vec{\Phi} \times \vec{n}] \cdot \vec{v}_{\sigma}\right) d S-\operatorname{Re} \frac{1}{\psi} \int_{\partial G}\left|\vec{v}_{\sigma}\right|^{2} d S,
\end{aligned}
$$

where $\vec{v}_{\sigma}=\vec{n} \times[\vec{v} \times \vec{n}]$ is the component of $\vec{v}$ tangent to $\partial G$ and $\vec{n}$ the unit outward normal. Then

$$
\begin{aligned}
\frac{d}{d t}\left\|u(\cdot, t) ; L_{2}(G)\right\|= & 2 \operatorname{Re} \int_{G}\left\langle u_{t}+A(\partial) u, u\right\rangle d x d y d z \\
& -2 \operatorname{Re} \frac{1}{\psi} \int_{\partial G}\left([\vec{\Phi} \times \vec{n}] \cdot \vec{v}_{\sigma}\right) d S+2 \operatorname{Re} \frac{1}{\psi} \int_{\partial G}\left|\vec{v}_{\sigma}\right|^{2} d S .
\end{aligned}
$$

Recalling that $\psi=a+i b$, we can estimate the right-hand side:

$$
\begin{aligned}
& \frac{d}{d t}\left\|u(\cdot, t) ; L_{2}(G)\right\|+2 \frac{|a|}{|\psi|} \cdot\left\|\vec{v}_{\sigma} ; L_{2}(\partial G)\right\|^{2} \\
& \quad \leq c\left\{\left\|M u ; L_{2}(G)\right\| \cdot\left\|u ; L_{2}(G)\right\|+\left\|\vec{\Phi} \times \vec{n} ; L_{2}(\partial G)\right\| \cdot\left\|\vec{v}_{\sigma} ; L_{2}(\partial G)\right\|\right\} .
\end{aligned}
$$

We integrate over $(-\infty, t)$, multiply the resulting identity by $e^{-2 \gamma t}$, and then integrate over $(-\infty,+\infty)$. Changing the order of integration, we obtain

$$
\begin{aligned}
\int_{-\infty}^{+\infty} & e^{-2 \gamma t}\left\|u(\cdot, t) ; L_{2}(G)\right\|^{2} d t+\frac{|a|}{\gamma|\psi|} \int_{-\infty}^{+\infty} e^{-2 \gamma t}\left\|\vec{v}_{\sigma}(\cdot, t) ; L_{2}(\partial G)\right\|^{2} d t \\
\leq & \frac{c}{2 \gamma} \int_{-\infty}^{+\infty} e^{-2 \gamma t}\left\|M u(\cdot, t) ; L_{2}(G)\right\| \cdot\left\|u(\cdot, t) ; L_{2}(G)\right\| d t \\
& +\frac{c}{2 \gamma} \int_{-\infty}^{+\infty} e^{-2 \gamma t}\left\|\vec{\Phi}(\cdot, t) ; L_{2}(\partial G)\right\| \cdot\left\|\vec{v}_{\sigma}(\cdot, t) ; L_{2}(\partial G)\right\| d t .
\end{aligned}
$$

Next, we have

$$
\begin{array}{r}
\int_{-\infty}^{+\infty} e^{-2 \gamma t}\left\|u ; L_{2}(G)\right\|^{2} d t+\frac{|a|}{\gamma|\psi|} \int_{-\infty}^{+\infty} e^{-2 \gamma t}\left\|\vec{v}_{\sigma} ; L_{2}(\partial G)\right\|^{2} d t \\
\leq \frac{c}{2 \gamma} \int_{-\infty}^{+\infty} e^{-2 \gamma t}\left(\left\|M u ; L_{2}(G)\right\|+\sqrt{\frac{|\psi| \gamma}{|a|}} \cdot\left\|\vec{\Phi} ; L_{2}(\partial G)\right\|\right) \\
\times\left(\left\|u ; L_{2}(G)\right\|+\sqrt{\frac{|a|}{\gamma|\psi|}} \cdot\left\|\vec{v}_{\sigma} ; L_{2}(\partial G)\right\|\right) d t
\end{array}
$$

By the Cauchy inequality,

$$
\begin{aligned}
& \int_{-\infty}^{+\infty} e^{-2 \gamma t}\left(\left\|u ; L_{2}(G)\right\|^{2}+\frac{|a|}{\gamma|\psi|} \cdot\left\|\vec{v}_{\sigma} ; L_{2}(\partial G)\right\|^{2}\right) d t \\
& \quad \leq \frac{c}{\gamma^{2}} \int_{-\infty}^{+\infty} e^{-2 \gamma t}\left(\left\|M u ; L_{2}(G)\right\|^{2}+\frac{|\psi| \gamma}{|a|} \cdot\left\|\vec{\Phi} ; L_{2}(\partial G)\right\|^{2}\right) d t
\end{aligned}
$$

Since $\zeta$ is an arbitrary function, application of Parseval's identity for the Fourier transform $\mathcal{F}_{t \rightarrow \tau}$ yields (56).

Our final remark concerns a global estimate for the problem adjoint with respect to the Green formula. 
Remark 2.9.3. Suppose $v=(\vec{u}, \vec{v}, h, q) \in C_{c}^{\infty}(G)$ satisfies the boundary conditions

$$
-\vec{n} \times[\vec{v} \times \vec{n}]+\bar{\psi}[\vec{n} \times \vec{u}]=[\vec{\Phi} \times \vec{n}], \quad h=0, \quad q=0
$$

on $\partial G$. Then

$$
\begin{aligned}
& \gamma^{2}\left\|v ; L_{2}(G)\right\|^{2}+(\gamma|a| /|\psi|) \cdot\left\|\vec{v} \times \vec{n} ; L_{2}(\partial G)\right\|^{2} \\
& \quad \leq c\left\{\left\|M\left(D_{x}, D_{y}, D_{z}, \bar{\tau}\right) v ; L_{2}(G)\right\|^{2}+(\gamma|\psi| /|a|) \cdot\left\|\vec{\Phi} \times \vec{n} ; L_{2}(\partial G)\right\|^{2}\right\} .
\end{aligned}
$$

Estimate (56) corresponds to the problem $\left\{\tau+A(D), \Gamma_{1}\right\}$, and (57) to the problem $\left\{\bar{\tau}+A(D), \Gamma_{2}\right\}$. The verification of (57) is similar to that of (56) . The only difference is that we must start with $\partial_{t}-A(\partial)$ and then obtain $\bar{\tau}+A(D)$.

\section{REFERENCES}

[1] B. A. Plamenevskiı̌, On the Dirichlet problem for the wave equation in a cylinder with edges, Algebra i Analiz 10 (1998), no. 2, 197-228; English transl., St. Petersburg Math. J. 10 (1999), no. 2, 373-397; Corrections, Algebra i Analiz 10 (1998), no. 3, 224. (Russian) MR1629407(99i:35087a) MR628054 (99i:35087b)

[2] A. Yu. Kokotov and B. A. Plamenevskiı̌, On the Cauchy-Dirichlet problem for hyperbolic systems in a wedge, Algebra i Analiz 11 (1999), no. 3, 140-196; English transl., St. Petersburg Math. J. 11 (2000), no. 3, 497-534. MR 1711368 (2000j:35170)

[3] A. Yu. Kokotov, P. Neйttaanmyaki, and B. A. Plamenevskiŭ, Problems of diffraction on a cone: asymptotic behavior of the solutions near the vertex, Zap. Nauchn. Sem. S.-Peterburg. Otdel. Mat. Inst. Steklov. (POMI) 259 (1999), 122-145; English transl., J. Math. Sci. 109 (2002), no. 5, 18941910. MR $1754360(2001 \mathrm{~m}: 35195)$

[4] - The Neumann problem for the wave equation in a cone, Function Theory and Applications, Probl. Mat. Anal., vyp. 20, S.-Peterburg. Gos. Univ., St. Petersburg, 2000, pp. 71-110; English transl., J. Math. Sci. 102 (2000), no. 5, 4400-4428. MF,1807064 (2002k:35180)

[5] - On the Neumann problem for hyperbolic systems in a wedge, Dokl. Akad. Nauk 383 (2002), no. 5, 608-611. (Russian) MR1930107 (2003e:35177)

[6] M. S. Agranovich and M. I. Vishik, Elliptic problems with a parameter and parabolic problems of general type, Uspekhi Mat. Nauk 19 (1964), no. 3, 53-161; English transl. in Russian Math. Surveys 19 (1964), no. 3. MR0192188 (33:415)

[7] M. Sh. Birman and M. Z. Solomyak, $L^{2}$-theory of the Maxwell operator in arbitrary domains, Uspekhi Mat. Nauk 42 (1987), no. 6 (258), 62-76; English transl., Russian Math. Surveys 42 (1987), no. 6, 75-96. MR0933995 (89e:35127)

[8] I. S. Gudovich, S. G. KreĬn, and I. M. Kulikov, Boundary value problems for the Maxwell equations, Dokl. Akad. Nauk SSSR 207 (1972), no. 2, 321-324; English transl., Soviet Phys. Dokl. 17 (1972), 1053-1055 (1973). MR0316892 (47:5440)

[9] S. I. Matyukevich, On a nonstationary Maxwell system in a wedge, Vestnik S.-Peterburg. Univ. Ser. 1 2000, vyp. 1, 35-43; English transl., Vestnik St. Petersburg Univ. Math. 33 (2000), no. 1, 16-22 (2001). MR1823725 (2002e:35225)

[10] M. Costabel and M. Dauge, Singularities of electromagnetic fields in polyhedral domains, Arch. Rational Mech. Anal. 151 (2000), 221-276. MF.1753704 (2002c:78005)

[11] V. A. Kozlov, V. G. Maz'ya, and J. Rossmann, Spectral problems associated with corner singularities of solutions to elliptic equations, Math. Surveys Monogr., vol. 85, Amer. Math. Soc., Providence, RI, 2001. MR1788991 (2001i:35069)

[12] S. A. Nazarov and B. A. Plamenevskiü, Elliptic problems in domains with piecewise smooth boundaries, "Nauka", Moscow, 1991. (Russian) MF1283387 (95h:35001)

[13] — Elliptic problems in domains with piecewise smooth boundaries, de Gruyter Exp. Math., vol. 13, Walter de Gruyter, Berlin, 1994. MR.1283387 (95h:35001) 
[14] A. Erdélyi, W. Magnus, F. Oberhettinger, and F. G. Tricomi, Tables of integral transforms. Vol. 1, McGraw-Hill Book Co., Inc., New York etc., 1954. MR0061695 (15:868a)

[15] V. S. Vladimirov, The equations of mathematical physics, "Nauka", Moscow, 1981; English transl., "Mir", Moscow, 1984. MR0764399 (86f:00030)

St. Petersburg State University, Physics Department, Division of Mathematical Physics, St. Petersburg, Russia

E-mail address: matsi@front.ru, matsi@math.nw.ru

Received 23/APR/2003

Translated by B. A. PLAMENEVSKII 